TRANSACTIONS OF THE

\title{
ERGODIC ATTRACTORS
}

\author{
CHARLES PUGH AND MICHAEL SHUB
}

\begin{abstract}
Using the graph transform method, we give a geometric treatment of Pesin's invariant manifold theory. Beyond deriving the existence, uniqueness, and smoothness results by Fathi, Herman, and Yoccoz our method allows us to do four things: optimally conserve smoothness, deal with endomorphisms, prove absolute continuity of the Pesin laminations, and produce ergodic attractors.
\end{abstract}

\section{INTRODUCTION}

In [FHY], Fathi, Herman, and Yoccoz present an exposition of Pesin's Stable Manifold Theorem [P1, P2]. We intended to take up where they left offabsolute continuity and ergodicity. To do so, we found it necessary to reprove some of their results using graph transform techniques. As a by-product of this approach, we are able to show that Pesin's stable manifolds are of class $C^{r}$ when $r \geq 2$ is an integer and the dynamics are only $C^{r}$. See $\S 6$. In $\S 5$, we present the Pesin theory for endomorphisms. In $\S 4$ we establish absolute continuity of the Pesin laminations and in $\S 2$ we use it to produce ergodic attractors.

Fix a diffeomorphism of a compact, smooth, boundaryless, Riemann $m$ manifold $f: M \rightarrow M$, and let $v \in T_{p} M, v \neq 0$, be given. The growth rate of $v$ under $T f^{n}$ is $\lambda$ provided that

$$
\lambda=\lim _{|n| \rightarrow \infty}\left|T f^{n}(v)\right|^{1 / n} .
$$

Thus, $\left|T f^{n}(v)\right| \sim \lambda^{n}|v|$ when $|n|$ is large. The set of all vectors in $T_{p} M$ with growth rate $\lambda, E_{p}^{\lambda}$, turns out to be a linear subspace of $T_{p} M$. We call it a Lyapunov space. It is automatic that the Lyapunov spaces are $T f$-invariant: $T f\left(E_{p}^{\lambda}\right)=E_{f p}^{\lambda}$.

Equivalent to the idea of growth rate is that of Lyapunov exponent. One says that $\chi$ is the Lyapunov exponent of $v$ if

$$
\chi(v)=\lim _{|n| \rightarrow \infty} \frac{1}{n} \log \left|T f^{n}(v)\right| .
$$

Received by the editors July 31, 1987 and, in revised form, November 12, 1987.

1980 Mathematics Subject Classification (1985 Revision). Primary 58Fxx; Secondary 58F10, $58 \mathrm{~F} 11,58 \mathrm{~F} 12,58 \mathrm{~F} 13,58 \mathrm{~F} 15,58 \mathrm{~F} 35$.

Both authors were partially supported by NSF grants; Charles Pugh was also supported by a British SERC grant. 
The vector $v$ has Lyapunov exponent $\chi$ if and only if it has growth rate $\lambda=e^{\chi}$. Generally, we use the growth rate concept. The basic properties of the Lyapunov spaces $E_{p}^{\lambda}$ are described in

Oseledec's Regularity Theorem. Given $f$ as above, there exists an $f$-invariant Borel set $\mathscr{R} \subset M$ and, for each $\varepsilon>0$, a Borel function $R_{\varepsilon}: \mathscr{R} \rightarrow(1, \infty)$ such that for all $v \in E_{p}^{\lambda}, p \in \mathscr{R}$, and $n \in \mathbb{Z}$,

(a) $\bigoplus_{\lambda} E_{p}^{\lambda}=T_{p} M$.

(b) $\left\{R_{\varepsilon}(p)(1+\varepsilon)^{|n|}\right\}^{-1} \leq\left|T_{p} f^{n}(v)\right| / \lambda^{n}|v| \leq\left\{R_{\varepsilon}(p)(1+\varepsilon)^{|n|}\right\}$.

(c) $\varangle\left(E_{p}^{\Lambda}, E_{p}^{\Lambda^{\prime}}\right) \geq R_{\varepsilon}(p)^{-1}$ if $\Lambda \cap \Lambda^{\prime}=\varnothing$.

(d) $(1+\varepsilon)^{-1} \leq R_{\varepsilon}(f p) / R_{\varepsilon}(p) \leq 1+\varepsilon$.

Moreover, $\mathscr{R}$ has "total probability" in the sense that $\mu(\mathscr{R})=1$ for every $f$ invariant Borel probability measure $\mu$ on $M$, and the growth rates and Lyapunov spaces are Borel functions of $p \in \mathscr{R}$.

(b) means that up to the factor in braces, $T f^{n}$ is a dilation of $E^{\lambda}$ by $\lambda^{n}$. The angle referred to in (c) is the smallest angle between a vector from $E_{p}^{\Lambda}=$ $\bigoplus_{\lambda \in \Lambda} E_{p}^{\lambda}$ and one from $E_{p}^{\Lambda^{\prime}}=\bigoplus_{\lambda \in \Lambda^{\prime}} E_{p}^{\lambda}$. It is asserted that these spaces stay perpendicular, up to the factor $R_{\varepsilon}$, as we iterate along the orbit. (d) means that $R_{\varepsilon}$ is slowly varying along the orbit [K]. Pesin calls the orbits in $\mathscr{R}$ regular. We call the function $R_{\varepsilon}$ a regularity function. One could paraphrase Oseledec's Theorem as:

Most orbits of $f$ are regular, and over a regular orbit $T f^{n}$ resembles the $n$th power of a square matrix. The resemblance is valid modulo a slowly varying fudge factor.

The proof of this truly remarkable theorem can be found in [O, R, or M2].

Definition. The points $p, y \in M$ are exponentially forward asymptotic if for some $C>0$ and some $\lambda, 0<\lambda<1$,

$$
d\left(f^{n} y, f^{n} p\right) \leq C \lambda^{n} \text { for all } n \geq 0 .
$$

The stable set of $p \in M$ is

$$
W^{s}(p)=\{y \in M: y \text { is exponentially forward asymptotic with } p\} .
$$

Clearly, any two stable sets are either equal or disjoint, so

$$
\mathscr{W}^{s}=\left\{W^{s}(p): p \in M\right\}
$$

partitions $M$. We call $\mathscr{W}^{s}$ the stable partition of $M$. Exponential reverse asymptoticity corresponds to $n \leq 0$ and the unstable set, $W^{u}(p)$, consists of all points which are exponentially reverse asymptotic with $p$. Correspondingly we have the unstable partition $\mathscr{W}^{u}$. (We are aware of the abuse of language here; it would be more accurate to refer to $W^{s}(p)$ as the "exponentially stable set" but we will not do so.) 
Let $\mathscr{O}$ be a regular orbit. If the growth rates of vectors $v \in T_{\mathscr{Q}} M$ are never equal to 1 then we say that $\mathscr{O}$ is weak hyperbolic. Its weak hyperbolic splitting is $E^{u} \oplus E^{s}$ where

$$
E_{p}^{u}=\bigoplus_{\lambda>1} E_{p}^{\lambda}, \quad E_{p}^{s}=\bigoplus_{\lambda<1} E_{p}^{\lambda} .
$$

The set of all weak hyperbolic orbits is denoted by $\mathscr{H}$. When $\mathscr{O}$ is a periodic orbit, weak hyperbolicity is the same as ordinary (or uniform) hyperbolicity, and the weak hyperbolic splitting reduces to the usual one. The same is true if $\mathscr{O}$ is part of some hyperbolic set. Just as in the uniform case, one understands the dynamics along an orbit via the stable manifold theorem.

Theorem 1: Pesin's Stable Manifold Theorem along one orbit. Suppose that the diffeomorphism $f$ is of class $C^{1+\beta}$ for some $\beta>\beta^{\prime}>0$. Let $\mathscr{O}$ be a weak hyperbolic orbit with splitting $E^{u} \oplus E^{S}$ and let $p \in \mathscr{O}$ be given. Through $f^{n} p$ pass $C^{1+\beta^{\prime}}$ discs $W_{n}^{u}$ and $W_{n}^{s}$ tangent to $E^{u}$ and $E^{s}$ at $f^{n} p$ such that the unstable and stable sets of $p$ are

$$
W^{u}(p)=\bigcup_{n \geq 0} f^{n}\left(W_{-n}^{u}\right), \quad W^{s}(p)=\bigcup_{n \geq 0} f^{-n}\left(W_{n}^{s}\right) .
$$

Moreover, $f$ overflows $\left\{W_{n}^{u}\right\}$ and underflows $\left\{W_{n}^{s}\right\}$ in the sense that for all $n \in \mathbb{Z} \quad f\left(W_{n}^{u}\right) \supset W_{n+1}^{u}$ and $f\left(W_{n}^{s}\right) \subset W_{n+1}^{s}$.

In short, the weak hyperbolic linear dynamics over $\mathscr{O}$ in $T M$ "integrate" to give corresponding weak hyperbolic, local, nonlinear dynamics along $\mathscr{O}$ in $M$. We are then justified in referring to $W^{u}(p)$ and $W^{s}(p)$ as unstable and stable manifolds since they are monotone unions of discs and such a union is always an injectively immersed Euclidean space [B]. See 3.8 for the proof of Theorem 1 , and also 3.17 for the proof of some center manifold theorems under similar hypotheses. In $\S 6$ we show how to make $\beta^{\prime}=\beta$. See 6.4 .

Next, we formulate the concept of absolute continuity of the unstable partition. Given $p_{0} \in \mathscr{H}$, the set of weak hyperbolic orbits, we draw small, smooth discs $D$ and $D^{\prime}$, which pass near $p_{0}$ and are quite transverse to $W^{u}\left(p_{0}\right)$. We look at those $p \in \mathscr{H}$ near $p_{0}$ whose unstable manifolds have the same dimension as $W^{u}\left(p_{0}\right)$ and which cross $D, D^{\prime}$ locally. See Figure 1. (The word "locally" suggests a lot of the weak uniformity of $\mathscr{W}^{u}$.) These intersections, $y=W^{u} \cap D$ and $y^{\prime}=W^{u} \cap D^{\prime}$, define a bijection $h$ from a subset of $D$ to one of $D^{\prime} ; h$ is called a $\mathscr{W}^{u}$-holonomy map, $h: y \mapsto y^{\prime}$.

Definition. $\mathscr{W}^{u}$ is absolutely continuous if its holonomy maps are always Lebesgue measurable and transform Lebesgue zero sets of $D$ to Lebesgue zero sets of $D^{\prime}$.

Theorem 2: Absolute continuity. $\mathscr{W}^{u}$ and $\mathscr{W}^{s}$ are absolutely continuous.

Theorem 2 is Pesin's main technical result. See $\S 4$ for its proof. As announced in [PS2], we use it to produce "ergodic attractors". 


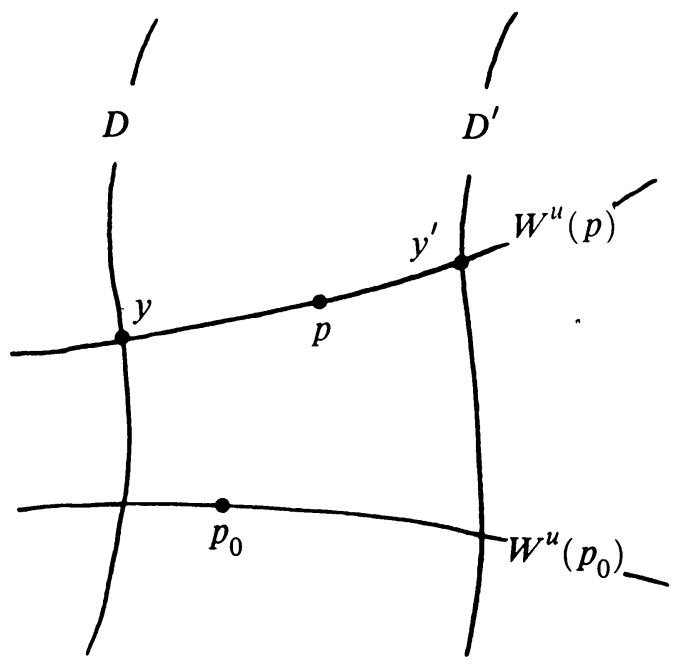

FIGURE 1. $\mathscr{W}^{u}$-holonomy

Definition. An ergodic attractor for $f$ is an $f$-invariant, Borel set $A \subset M$ together with an $f$-invariant, Borel probability measure $\nu$ on $A$ such that for some set $W \subset M$ with positive Lebesgue measure

(EAi) Each $w \in W$ is attracted toward $A$, $\operatorname{dist}\left(f^{n} w, A\right) \rightarrow 0$ as $n \rightarrow \infty$.

(EAii) $\nu$ is $f$-ergodic.

(EAiii) For every continuous function $\phi: M \rightarrow \mathbf{R}$ and almost every point $w \in W$ (respecting Lebesgue measure), the Birkhoff average of $\phi$ along the positive $f$-orbit through $w$ converges to $\int_{A} \phi d \nu$,

$$
\mathscr{B}_{+}(\phi, w) \stackrel{\text { def }}{=} \lim _{n \rightarrow \infty} \frac{1}{n} \sum_{j=1}^{n} \phi\left(f^{j} w\right)=\int_{A} \phi d \nu .
$$

Theorem 3: Existence of ergodic attractors. Suppose that the diffeomorphism $f: M \rightarrow M$ is of class $C^{1+\beta}, \beta>0$, and $\mu$ is an $f$-invariant, Borel, probability measure such that

(i) $f$ has almost no zero Lyapunov exponent respecting $\mu$; i.e., $\mu(\mathscr{H})=1$ where $\mathscr{H}$ is the set of weak hyperbolic orbits.

(ii) $\mu$ induces a conditional measure on the local unstable manifold of $\mu$ almost-every $p \in \mathscr{H}$, which is absolutely continuous with respect to its natural Riemann measure.

Then, up to a set of $\mu$-measure zero, $\mathscr{H}$ is the countable union of ergodic attractors $A_{i}$, and the ergodic measure $\nu_{i}$ on $A_{i}$ is the normalization of $\mu$. In fact, somewhat more is true: the basin of attraction of $A_{i}$ is the union of the stable sets of the points in $A_{i}, W=W^{s} A_{i}$, and the Birkhoff average converges for every $w \in W^{s} A_{i}$. 
Corollary: Pesin's Ergodic Component Theorem. If $f$ preserves Lebesgue measure and almost every orbit is weak hyperbolic then, up to a zero set, $M$ is the countable union of ergodic attractors. In particular, ergodic attractors exist.

Remarks. The manner in which $\mu$-induces measures on the local unstable manifolds-disintegration-is explained in the proof. See $\S 2$. Recall that a measure $\nu$ is absolutely continuous respecting a measure $\rho, \nu \ll \rho$, if every zero set of $\rho$ is a zero set of $\nu$. "Countable" means finite or countably infinite.

It is always true that $M$ can be partitioned into ergodic components $C_{\alpha}$ in a way that disintegrates the measure $\mu$ onto the $C_{\alpha}$, but in general there are uncountably many $C_{\alpha}$. See [M2]. For example, if $f: S^{2} \rightarrow S^{2}$ is an irrational rotation then, up to a zero set, the $C_{\alpha}$ are the two poles and the uncountably many latitude circles. Although $f$ preserves Lebesgue measure, none of its orbits is weak hyperbolic.

One might ask: when is the number of ergodic components finite? In Pesin's Ergodic Component Theorem, if $\mathscr{H}$ is compact then all of $M$ is a single ergodic attractor becasuse $f$ is Anosov [M3]. It would be of interest to know in what other circumstances the number of ergodic components is finite

\section{ERgodic ATTRACTORS EXIST}

In this section we prove that Theorem 2 (absolute continuity) implies Theorem 3 (ergodic attractors). Let $G_{ \pm}$and $G$ be the sets of points $p \in M$ such that for all continuous functions $\phi: M \rightarrow \mathbf{R}$, the three Birkhoff averages

$$
\begin{aligned}
& \mathscr{B}_{ \pm}(\phi, p) \stackrel{\text { def }}{=} \lim _{n \rightarrow \pm \infty} \frac{1}{|n|+1} \sum_{j=0}^{n} \phi\left(f^{j} p\right), \\
& \mathscr{B}(\phi, p) \stackrel{\text { def }}{=} \lim _{n \rightarrow \infty} \frac{1}{2 n+1} \sum_{j=-n}^{n} \phi\left(f^{j} p\right)
\end{aligned}
$$

exist and are equal. By [P'n], $G \cap G_{-} \cap G_{+}=G^{*}$ is of full $\mu$-measure and is $f$-invariant. Also, continuity of $\phi$ implies that $G_{+}$consists of whole stable sets while $G_{-}$consists of whole unstable ones.

The set $\mathscr{H}$ is the monotone union $\bigcup_{\tau>1} \mathscr{H}(\tau)$ where $\mathscr{H}(\tau)=\{p \in \mathscr{H}:$ no growth rate along the orbit through $p$ lies in the interval $\left.\left[\tau^{-1}, \tau\right]\right\}$. For any $\tau>1$, choose $\varepsilon, 0<\varepsilon \ll \tau-1$, and determine a regularity function $R_{\varepsilon}$ from Oseledec's Theorem. Define $\mathscr{H}(\tau, N)=\left\{p \in \mathscr{H}: R_{\varepsilon}(p) \leq N\right\}$. Since $\mathscr{H}=\bigcup \mathscr{H}(\tau, N)$ has $\mu$-measure $1, \mathscr{H}$ can be divided into countably many hyperbolic blocks $P$ of positive $\mu$-measure: each $P$ is a small subset of some $\mathscr{H}(\tau)$ on which $R_{\varepsilon}$ is bounded. See $\S 3$, especially 3.2 and 3.6.

Take any point $p_{0}$ in such a $P$. By Theorem 1 (see also Theorem 3.8), there exists a neighborhood $U$ of $p_{0}$, diffeomorphic to a product of discs, $U \approx U^{u} \times U^{s}$, and a compact subneighborhood $U_{0}$ of $p_{0}$ such that the $U$ local unstable manifolds through points of the closure of $P \cap U_{0}$ are graphs of maps $U^{u} \rightarrow U^{s}$ with small Lipschitz constant. They depend continuously on 
$p$ and are disjoint or equal. Dually, the $U$-local stable manifolds are graphs of maps $U^{s} \rightarrow U^{u}$ with small Lipschitz constant, they depend continuously on $p$, and they are disjoint or equal. This gives a continuous product structure. The unique intersection point $W_{U}^{u}(p) \cap W_{U}^{s}(q)$ need not lie in $P$, nor even in $\mathscr{H}$, but it does exist if $p, q$ lie in the closure of $P \cap U_{0}$. See Figure 2.

Without loss of generality we replace $P$ by $P \cap U_{0} \cap G^{*}$, for $\mu\left(M \backslash G^{*}\right)=0$ and $P \cap U_{0}$ is just a smaller version of $P$.

Now we discuss disintegration. It is a kind of Fubini process, valid in great generality [B'i]. The local unstable manifold $W_{U}^{u}(p)$ is a Borel function of $p \in \bar{P}$. (In fact it is continuous). This implies that $\mu$ disintegrates to measures $\bar{\mu}_{p}$ on the $W_{U}^{u}(p), p \in \bar{P}$ [B'i, pp. 58-59]. They are Borel measures and they satisfy a Fubini equation

$$
\mu(S)=\int_{\vec{P}} \bar{\mu}_{p}(S) d \eta,
$$

where $S$ is any measurable subset of $\bigcup_{p \in P} W_{U}^{u}(p)$ and $\eta$ is the "push ahead" measure on $\bar{P}$. That is, $\eta(A)=\mu\left(\bigcup_{a \in A} W_{U}^{u}(a)\right)$ for measurable subsets $A \subset \bar{P}$.

To be more accurate, $\bar{\mu}_{p}$ depends on $W_{U}^{u}(p)$, not on $p$. To avoid this abuse of notation, let us re-index $W_{U}^{u}(p)$ by its unique intersection with $W_{U}^{s}\left(p_{0}\right)$. See Figure 2. Thus, if $y=W_{U}^{u}(p) \cap W_{U}^{s}\left(p_{0}\right)$ then we write $W_{y}$ for $W_{U}^{u}(p)$ and $\bar{\mu}_{y}$ for $\bar{\mu}_{p}$. The space $\bar{Y} \subset W_{U}^{s}\left(p_{0}\right)$ of these $y$ is compact and supports the measure $\eta$. Let $\mu_{y}$ be the restriction of $\bar{\mu}_{y}$ to $W_{y} \cap P$, and call $Y=\{y \in$ $\bar{Y}: W_{y}=W_{U}^{u}(p)$ for some $\left.p \in P\right\}$. In fact, by continuity of $W_{U}^{u}(p)$ for $p \in P, \bar{Y}$ is the closure of $Y$. The Fubini equation $\mu(S)=\int_{Y} \mu_{y}(S) d \eta$ holds for measurable subsets $S \subset \bigcup_{y \in Y} W_{y}$.

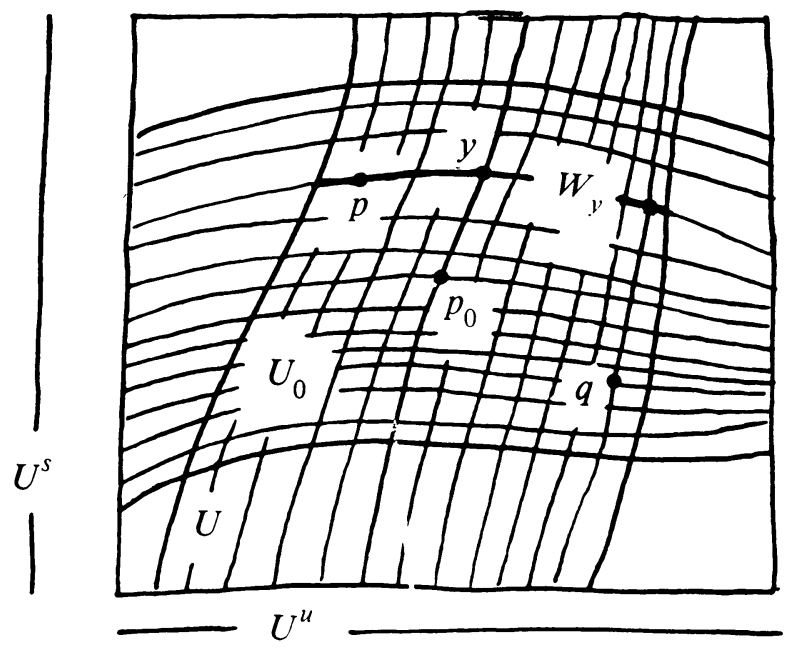

FIGURE 2. The product neighborhood of $p_{0}$ 
Assumption (ii) in Theorem 3 says that $\mu_{y} \ll \rho_{y}$ where $\rho_{y}$ is the smooth Riemann measure on $W_{y}$. Consider the subset $P_{y} \subset W_{y}$ where the Radon Nikodym derivative $d \mu_{y} / d \rho_{y}$ is positive. ${ }^{1}$ On $P_{y}, \mu_{y}$ and $\rho_{y}$ are equivalent measures. Without loss of generality we may assume that $P_{y}=P \cap W_{y}$. For if $Z$ is the subset of $P$ on which $d \mu_{y} / d \rho_{y}=0$, then $P \backslash Z$ is a hyperbolic block and by the Fubini equation, $\mu(P)=\mu(P \backslash Z)$.

Inside $P$ we produce sets $E_{i}$ and iterate them to get ergodic attractors as follows. Let $\mathscr{P}=\left\{\boldsymbol{P}_{y}\right\}$ and define a relation on $\mathscr{P}$ according to local product structure:

$$
P_{y_{1}} \sim P_{y_{2}} \text { if } W_{U}^{s}\left(p_{1}\right)=W_{U}^{s}\left(p_{2}\right) \text { for some } p_{1} \in P_{y_{1}}, p_{2} \in P_{y_{2}} .
$$

Enlarge $\sim$ to an equivalence relation by forcing transitivity. See Figure 3. Let $h$ project $P$ into $W_{U}^{u}\left(p_{0}\right)$ by sliding along $\left\{W_{U}^{s}(p)\right\}_{p \in P}$. It carries equivalence classes into disjoint sets, each of which has positive Riemann measure. For $\mu_{y}\left(P_{y}\right)>0$ implies $\rho_{y}\left(P_{y}\right)>0$, and absolute continuity of the $\mathscr{W}^{s}$-holonomy map $W_{U}^{u}(p) \rightarrow W_{U}^{u}\left(p_{0}\right)$ (Theorem 2) then implies that $\rho_{y_{0}}\left(h\left(P_{y}\right)\right)>0$ for all $P_{y} \in \mathscr{P}$. Therefore, there are only countably many equivalence classes, say $E_{1}, E_{2}, \ldots$, and $\mu(P)=\sum \mu\left(E_{i}\right)$. Discard those $E_{i}$ with $\mu\left(E_{i}\right)=0$.

Set $A_{i}=\operatorname{Saturate}\left(E_{i}\right)=\bigcup_{n \in \mathbf{Z}} f^{n}\left(E_{i}\right)$. Since $E_{i}$ consists of whole $P_{y}$ 's, each of which has positive $\rho_{y}$-measure, $W^{s}\left(E_{i}\right)$ has positive Lebesgue measure by absolute continuity of $\mathscr{W}^{s}$ (Theorem 2); thus, $W^{s} A_{i}$ has positive Lebesgue measure, verifying (EAi) with $W=W^{s} A_{i}$. Also note that $A_{i} \subset \mathscr{H}$ since $E_{i} \subset P \subset \mathscr{H}$ and $\mathscr{H}$ is $f$-invariant.

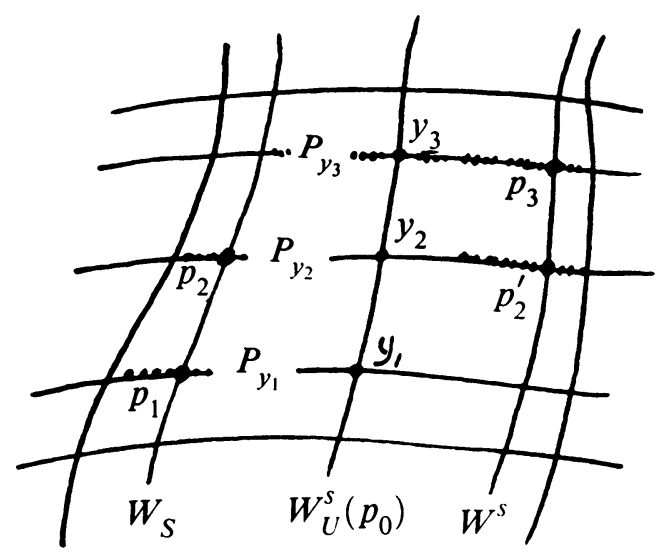

$$
d \mu_{y} / d \rho_{y}>0 \text { at } p_{1}, p_{2}, p_{2}^{\prime}, p_{3}
$$

FIGURE 3. The equivalence relation generated by $\sim$

\footnotetext{
${ }^{1}$ In fact, as pointed out to us recently by F. Ledrappier, one can show that $P_{y}=W_{y}$ by using the Jacobian estimates in $\S 4$.
} 
Since $E_{i} \subset G^{*}$ and $G^{*}$ is $f$-invariant, $A_{i} \subset G^{*}$. For every continuous function $\phi$ on $M$, the three Birkhoff averages $\mathscr{B}_{-}(\phi, a), \mathscr{B}_{+}(\phi, a)$, and $\mathscr{B}(\phi, a)$ exist at all points $a \in A_{i}$ and are equal. Clearly, by continuity, $\mathscr{B}_{-}(\phi, x)$ is constant on any $P_{y}$, and $\mathscr{B}_{+}\left(\phi, x_{1}\right)=\mathscr{B}_{+}\left(\phi, x_{2}\right)$ for any $x_{1} \in P_{y_{t}}, x_{2} \in P_{y_{2}}$ with $P_{y_{1}} \sim P_{y_{2}}$. Hence,

For all continuous $\phi$ on $M, \mathscr{B}_{-}(\phi, a), \mathscr{B}_{+}(\phi, a)$, and $\mathscr{B}(\phi, a)$

(*) are the same constant function $\mathscr{B}(\phi)$ on $A_{i}$. Moreover, $\mathscr{B}_{+}(\phi, z)$ $=\mathscr{B}(\phi)$ for all $z \in W^{s} A_{i}$.

Birkhoff averaging gives a continuous, onto projection

$$
\mathscr{B}: \mathscr{L}^{1}(A, \nu) \rightarrow \operatorname{Inv}(f, \nu)
$$

where $\operatorname{Inv}(f, \nu)$ are the $f$-invariant $\nu$-integrable functions. Also,

$$
\int \mathscr{B}(\psi, x) d \nu=\int \psi d \nu
$$

See [P'n]. Take any $\psi \in \mathscr{L}^{1}\left(A_{i}, \nu_{i}\right)$ where $\nu_{i}$ is the normalization of $\mu \mid A_{i}$. Think of $\nu_{i}$ as a Borel measure on $M$. Then $\psi$ can be $\mathscr{L}^{1}$-approximated by a continuous $\phi$ on $M$. Since the $\mathscr{B}$-image of a dense set of such functions is contained in the set of constant functions, the entire $\mathscr{B}$-image is contained in the set of constant functions. In particular, $\operatorname{Inv}\left(f, \nu_{i}\right)=$ Constants, so $f$ is $\nu_{i}$-ergodic, verifying (EAii). Since the constant is $\int \psi d \nu_{i}$, (EAiii) is also verified.

The equivalence classes $E_{i}$ exhaust $P$ up to a set of $\mu$-measure zero. Thus, $P$ is contained in the union of countably many ergodic attractors, and all of them are contained in $\mathscr{H}$. Since $\mathscr{H}$ is the countable union of such hyperbolic blocks, and each hyperbolic block gives rise to only a countable number of ergodic attractors, we see that, up to a set of $\mu$-measure zero, $\mathscr{H}$ is a countable union of ergodic attractors. Q.E.D.

Proof of Pesin's Ergodic Component Theorem. Lebesgue measure, when disintegrated onto the local unstable manifolds $W_{y}$, satisfies $d \mu_{y} / d \rho_{y}>0$ almost everywhere. For the Lebesgue measure of open sets is positive and by the Fubini equation this must be reflected on most of the $W_{y}$. This verifies (ii) in Theorem 3. By assumption, almost all orbits are weak hyperbolic, which is (i), and so, up to a zero set, $\mathscr{H}$ is the union of ergodic attractors $A_{1}, A_{2}, \ldots$. Since $M \backslash \mathscr{H}$ is a zero set, the proof is complete. Q.E.D.

Remark. When $d \mu_{y} / d \rho_{y}>0$ almost everywhere (as is the case above) only one equivalence class $E$, and only one ergodic attractor $A$ arise from each hyperbolic block $P$ of positive measure.

\section{The Pesin Stable Manifold Theorem}

In this section, we investigate the local dynamics along weak hyperbolic orbits. We will do so not only along a single orbit (as in Theorem 1 in §1) but also 
along all the orbits passing through a "hyperbolic block" as described below. This lets us avoid many Borelian considerations. It also lets us estimate what weak continuity exists in the unstable and stable partitions $\mathscr{W}^{u}$ and $\mathscr{W}^{s}$.

First, we discuss the meaning of Oseledec regularity. Let $T_{z} M=\bigoplus_{\lambda} E_{z}^{\lambda}$ be the Lyapunov splitting along a regular orbit. For any $\Lambda \subset \mathbb{R}$, we set $E_{z}^{\Lambda}=$ $\bigoplus_{\lambda \in \Lambda} E_{z}^{\lambda}$. According to the definition of regularity, the angle between these $E_{z}^{\Lambda}, \varangle(z)=\min \left\{\varangle\left(E_{z}^{\Lambda}, E_{z}^{\Lambda^{\prime}}\right): \Lambda \cap \Lambda^{\prime}=\varnothing\right\}$ is bounded below by $R_{\varepsilon}(z)^{-1}$ where $R_{\varepsilon}$ is a regularity function. Thus,

$$
\varangle\left(f^{n} z\right) \geq\left(R_{\varepsilon} f^{n} z\right)^{-1} \geq\left(R_{\varepsilon}(z)(1+\varepsilon)^{|n|}\right)^{-1} .
$$

On the other hand, vectors in $E_{z}^{\lambda}$ are dilated by the factor $\lambda^{n}$, up to (at worst) a slowly growing factor. That is,

$$
\left(R_{\varepsilon}(z)(1+\varepsilon)^{|n|}\right)^{-1} \lambda^{n} \leq\left|T f^{n}(v)\right| /|v| \leq R_{\varepsilon}(z)(1+\varepsilon)^{|n|} \lambda^{n}
$$

To combine these estimates from several different Lyapunov spaces we use the following simple lemma from linear algebra.

3.1. Lemma. Suppose that $\pi: U \supset$ is a projection of the inner product space $U$ into itself, $\pi^{2}=\pi$. Let $\alpha$ be the infimum of the angles between nonzero vectors in the range and kernel of $\pi$. Then $\|\pi\|=\csc \alpha$.

Proof. Express any unit vector $u \in U$ as $v+w$ where $v=\pi u$ and $w=u-\pi u$. Clearly, $v \in \operatorname{range}(\pi), w \in \operatorname{kernel}(\pi)$. Call $E^{\prime}=\operatorname{span}(v, w)$. If $\operatorname{dim}\left(E^{\prime}\right) \neq 2$ then $v=0$ or $w=0$ and $|\pi u|=0$ or $|\pi u|=1$ respectively. If $\operatorname{dim}\left(E^{\prime}\right)=2$ then from Figure 4 it is clear that $|v| \leq\left(\sin \alpha^{\prime}\right)^{-1}$ where $\alpha^{\prime}$ is the angle between $v$ and $w$. In either case, $|\pi u| \leq \csc \alpha$. The reverse inequality is equally easy to prove. Q.E.D.

Now take $\Lambda=[\alpha, \beta]$ and choose an interval $[a, b]$ containing $[\alpha, \beta]$ in its interior. Thus, $0<a<\alpha<\beta<b<\infty$.

3.2. Proposition: Growth Control. Given $\varepsilon>0$, there exists a regularity function $G_{\varepsilon}: \mathscr{R} \rightarrow(1, \infty)$ such that for all $v \in E_{z}^{\Lambda}$

$$
\begin{array}{ll}
G_{\varepsilon}(z)^{-1} a^{n}|v| \leq\left|T f^{n}(v)\right| \leq G_{\varepsilon}(z) b^{n}|v|, & n \geq 0, \\
G_{\varepsilon}(z)^{-1} b^{n}|v| \leq\left|T f^{n}(v)\right| \leq G_{\varepsilon}(z) a^{n}|v|, & n \leq 0 .
\end{array}
$$

Proof. Let $\pi^{\lambda}: T_{R} M \rightarrow E^{\lambda}$ be the projection whose kernel is the sum of the Lyapunov spaces $E^{\mu}$ with $\mu \neq \lambda$. Since the Lyapunov splitting is $T f$-invariant, $T f$ and $\pi^{\lambda}$ commute. Choose $\delta>0$ such that $\delta<\varepsilon, \delta<1, a(3 \delta+1)^{2} \leq \alpha$, $\beta \leq b(3 \delta+1)^{-2}$. Thus, $\delta \ll \min \{\alpha-a, b-\beta\}$. Let $R_{\delta}: \mathscr{R} \rightarrow(1, \infty)$ be a regularity function. If $n \geq 0$ then for all $v \in E_{z}^{\Lambda}$,

$$
\left|T f^{n}(v)\right| \leq \sum_{\lambda \in \Lambda} R_{\delta}(z) \beta^{n}|| \pi^{\lambda} \||v| \leq 2 R_{\delta}(z)^{2} \operatorname{dim}\left(E^{\Lambda}\right) b^{n}|v|
$$




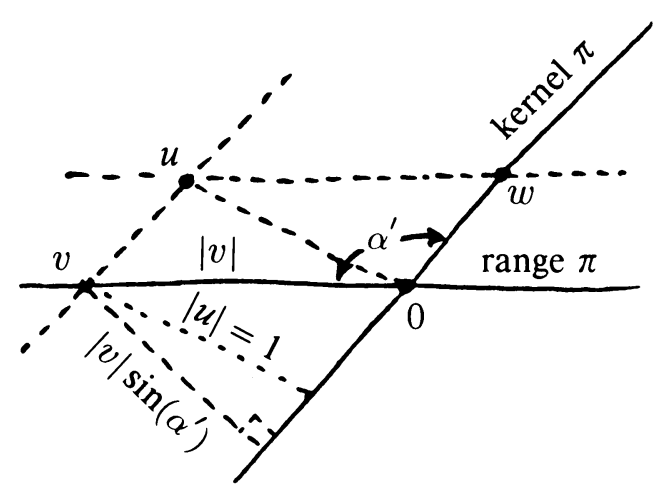

FIGURE 4. The range and kernel of $\pi$

Similarly, if $w \in E_{f^{n} z}^{\Lambda}$, then $\left|T f^{-n}(w)\right| \leq 2 R_{\delta}\left(f^{n} z\right)^{2} \operatorname{dim}\left(E^{\Lambda}\right) \alpha^{-n}|w|$. Taking $w=T f^{n}(v)$ gives

$$
\left|T f^{n}(v)\right| \geq\left(2 R_{\delta}(z)^{2} \operatorname{dim}\left(E^{\Lambda}\right)\right)^{-1} a^{n}|v| .
$$

Note that the factor $(1+\delta)^{2 n}$ arising from the comparison of $R_{\delta}(z)^{2}$ with $R_{\delta}\left(f^{n} z\right)^{2}$ is absorbed by changing $\alpha^{n}$ to $a^{n}$ because $\delta \ll \alpha-a$. The growth control estimate (GC.1) is then valid with

$$
G_{\varepsilon}(z)=2 R_{\delta}(z)^{2} \operatorname{dim}\left(E^{\Lambda}\right) .
$$

The square of the $\delta$-slowly varying function $R_{\delta}$ is a $3 \delta$-slowly varying function, for $(1+\delta)^{2} \leq 1+3 \delta$ when $\delta \leq 1$. A constant multiple of a $\delta$-slowly varying function is also $\delta$-slowly varying. Thus, $G_{\varepsilon}$ is $\varepsilon$-slowly varying, and since it is $>R_{\delta}$, it too is a regularity function. If $n \leq 0$ then $\delta \ll b-\beta$ implies (GC.2). Q.E.D.

When two functions are bounded on exactly the same subsets of their domains, such as $G_{\varepsilon}$ and $R_{\delta}$ are, we say they are comparable. Let us apply these ideas to the weak hyperbolic splitting $E^{u} \oplus E^{s}$. For $\tau>1$, let

$$
\mathscr{H}(\tau)=\left\{z \in \mathscr{R}: \text { no vector in } T_{z} M \text { has growth rate in }\left[\tau^{-1}, \tau\right]\right\} .
$$

Fix $1<a<\tau$ and $b=\max \left\{\sup \|T f\|, \sup \left\|T f^{-1}\right\|\right\}$. Call $T^{u} f$ and $T^{s} f$ the restrictions of $T f$ to $E^{u}$ and $E^{s}$. Modulo a growth control function $G_{\varepsilon}$, the growths of $T^{u} f^{n}$ and $T^{s} f^{n}$ over $\mathscr{H}(\tau)$ are controlled by $[a, b]$ and $\left[b^{-1}, a^{-1}\right]$ respectively. The $\varepsilon$ can be taken as small as desired.

The manifold $M$ comes equipped with a smooth Riemann structure but to prove Pesin's Stable Manifold Theorem we need to adapt it to the weak hyperbolic splitting $E^{u} \oplus E^{s}$. We will do so over $\mathscr{H}(\tau)$. Let $\langle$,$\rangle and ||$ denote the inner product and norm associated to the original Riemann structure. The new, or adapted, ones will be denoted by $\langle,\rangle^{*}$ and ||$^{*}$. They are defined 
as follows. Choose a constant $\mu, 1<\mu<a<\tau$ and write down a series definition of the inner product $\langle,\rangle^{*}$ on $T_{z} M$ for $z \in \mathscr{H}(\tau)$.

$$
\begin{aligned}
& \left\langle v, v^{\prime}\right\rangle^{*}=\sum_{n \geq 0} \mu^{2 n}\left\langle T f^{-n}(v), T f^{-n}\left(v^{\prime}\right)\right\rangle \quad \text { if } v, v^{\prime} \in E_{z}^{u}, \\
& \left\langle v, v^{\prime}\right\rangle^{*}=\sum_{n \geq 0} \mu^{2 n}\left\langle T f^{n}(v), T f^{n}\left(v^{\prime}\right)\right\rangle \quad \text { if } v, v^{\prime} \in E_{z}^{s}, \\
& \left\langle v, v^{\prime}\right\rangle^{*}=0 \text { if } v \in E_{z}^{u}, v^{\prime} \in E_{z}^{s} \text { or vice versa. }
\end{aligned}
$$

3.3. Proposition: Adapted Finsler. The series above converge. The induced Finsler ||$^{*}$ on $T_{\mathscr{H}(\tau)} M$ is Borel and has the following properties:

(a) For any $\varepsilon>0$ and any $\frac{1}{2} \varepsilon$-slowly varying growth control function $G$, there exists an $\varepsilon$-slowly varying Borel function $B_{\varepsilon}: \mathscr{H}(\tau) \rightarrow(1, \infty)$ that is comparable to $G$ and satisfies

$$
\sqrt{\frac{1}{2}}|w| \leq|w|^{*} \leq B_{\varepsilon}(z)|w|, \quad w \in T_{z} M, z \in \mathscr{H}(\tau) .
$$

(b) $\left\|T_{z} f\right\|^{*}$ and $\left\|T_{z} f^{-1}\right\|^{*}$ are uniformly bounded for $z \in \mathscr{H}(\tau)$.

(c) $T_{\mathscr{H}(\tau)} f$ is uniformly hyperbolic respecting $E^{u} \oplus E^{S}$ equipped with the adapted Finsler ||$^{*}$; i.e., for all $z \in \mathscr{H}(\tau)$,

$$
\left\|T_{z}^{s} f\right\|^{*}<\mu^{-1}<1<\mu<\left\|T_{z}^{u} f^{-1}\right\|^{*-1} \text {. }
$$

Proof. This is much the same as Proposition 4 of [FHY]. For completeness we present the proof anyway. By $3.2 \frac{1}{2} \varepsilon$-slowly varying growth control functions $G$ do exist. The series are dominated by $\sum_{n \geq 0}(\mu / a)^{2 n} G(z)|v|\left|v^{\prime}\right|$. Thus, the series converge, the adapted Finsler \|\|$^{*}$ exists and is Borel. By \|\|$^{*}$ we mean the operator norm respecting the Finsler ||$^{*}$. If $v \in E_{z}^{s}$ and $z \in \mathscr{H}(\tau)$ then

$$
|T f(v)|^{* 2}=\sum_{n \geq 0} \mu^{2 n}\left|T f^{n+1}(v)\right|^{2} \leq \mu^{-2}|v|^{* 2},
$$

so $\left\|T^{s} f\right\|^{*} \leq \mu^{-1}$. Similarly, $\left\|T^{u} f^{-1}\right\|^{*} \leq \mu^{-1}$, verifying (c). If $v \in E_{z}^{s}$ and $z \in \mathscr{H}(\tau)$ then

$$
|v|^{*} \leq\left\{\sum_{n \geq 0} \mu^{2 n}\left\|T_{z}^{s} f^{n}\right\|^{2}|v|^{2}\right\}^{1 / 2} \leq G(z) \sum_{n \geq 0}\left(\frac{\mu}{a}\right)^{n}|v|=G(z) a|v| /(a-\mu) .
$$

The same inequality holds if $v \in E_{z}^{u}$. The general vector $w=v+v^{\prime}$ with $v \in E_{z}^{u}$ and $v^{\prime} \in E_{z}^{s}$ satisfies

$$
\left\{|v|^{2}+\left|v^{\prime}\right|^{2}\right\}^{1 / 2} \leq \sqrt{2}|w| / \sin \left(E_{z}^{u}, E_{z}^{s}\right) \leq 2 \sqrt{2} G(z)|w|
$$

and so

$$
|w|^{*}=\left\{|v|^{* 2}+\left|v^{\prime}\right|^{* 2}\right\}^{1 / 2} \leq 4 G(z)^{2} a|w| /(a-\mu) .
$$


As in 3.2 , the bound $B_{\varepsilon}=4 G(z)^{2} a /(a-\mu)$ is $\varepsilon$-slowly varying since $G$ is $\frac{1}{3} \varepsilon$-slowly varying; and $|w|^{*} \leq B_{\varepsilon}(z)|w|$. When $G$ is bounded, so is $B_{\varepsilon}$. The estimate on $|w|^{*}$ from below is easy:

$$
|w| \leq|v|+\left|v^{\prime}\right| \leq|v|^{*}+\left|v^{\prime}\right|^{*} \leq \sqrt{2}|w|^{*}
$$

since $|v|^{2}$ and $\left|v^{\prime}\right|^{2}$ occur in the series defining $|v|^{* 2}$ and $\left|v^{\prime}\right|^{* 2}$. This completes the proof of (a).

Just as in [FHY], we claim

$$
\|T f\|^{* 2} \leq 2\|T f\|^{2}+5
$$

For if $v \in E_{z}^{u}$ then

$$
\begin{aligned}
\left|T_{z} f(v)\right|^{* 2} & =\sum_{n \geq 0} \mu^{2 n}\left|T_{f z} f^{-n}\left(T_{z} f(v)\right)\right|^{2}=\left|T_{z} f(v)\right|^{2}+\mu^{2}|v|^{* 2} \\
& \leq\left\|T_{z} f\right\|^{2}|v|^{2}+4|v|^{* 2} \leq\left\{2\left\|T_{z} f\right\|^{2}+4\right\}|v|^{* 2} .
\end{aligned}
$$

Combining this inequality, the fact from (c) that $\left\|T_{z}^{s} f\right\|^{*} \leq \mu^{-1}<1$, and $E^{u} \perp E^{s}$ respecting $\langle,\rangle^{*}$, we get

$$
\begin{aligned}
\left|T_{z} f(w)\right|^{* 2} & =\left|T_{z} f(v)\right|^{* 2}+\left|T_{z} f\left(v^{\prime}\right)\right|^{* 2} \leq\left\{2\left\|T_{z} f\right\|^{2}+4\right\}|v|^{* 2}+\left|v^{\prime}\right|^{* 2} \\
& \leq\left\{2\left\|T_{z} f\right\|^{2}+5\right\}|w|^{* 2},
\end{aligned}
$$

which verifies $(*)$ and half of $(b)$. The other half of $(b)$ is proved symmetrically. Q.E.D.

The next result is similar to Proposition 7 of [FHY]. We give its proof because it is exactly here that Pesin's extra $C^{1+\beta}$ smoothness assumption makes an appearance. If $f$ is $C^{1}$ but not $C^{1+\beta}$ for $\beta>0$, then 3.4 and 3.5 can fail, causing the breakdown of Pesin's theory. See [Pu]. By $\bar{f}$ we will refer to the map $f$, locally lifted to the tangent bundle via the smooth exponential associated to the original Riemann structure,

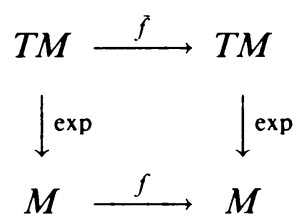

Rescaling $\langle$,$\rangle , we may assume that \exp _{z}$ embeds each $T_{z} M(R), \sup _{z}\left\|T_{z} f\right\|$ $<R$, and

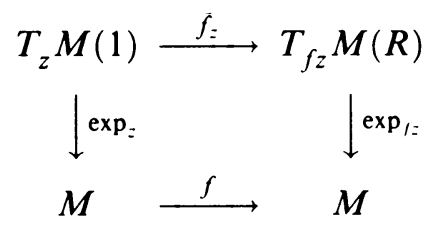

commutes; $\bar{f}_{z}$ is the restriction of $\bar{f}$ to $T_{z} M$. There is no real distinction between $f$ and $\bar{f} ; \bar{f}$ is merely the expression of $f$ in the exponential charts. 
3.4. Proposition: $C^{1}$ uniformity of $\bar{f}$. Suppose that the diffeomorphism $f: M$ $\rightarrow M$ is of class $C^{1+\beta}$ for some $\beta>0$. Let $\varepsilon, \nu>0$ be given. Then there exists an $\varepsilon$-slowly varying Borel "radius function" $r_{\varepsilon}: \mathscr{H}(\tau) \rightarrow(0,1)$ such that $\left\|\left(D \bar{f}_{z}\right)_{v}-T_{z} f\right\|^{*} \leq \nu$, for all $z \in \mathscr{H}(\tau)$ and all $v \in T_{z} M$ with $|v|^{*} \leq r_{\varepsilon}(z)$.

Proof. Since $f$ is $C^{1+\beta}$ on $M$ and $M$ is compact, $D \bar{f}$ is uniformly Hölder. There is a constant $C$ such that for all $v, v^{\prime} \in T_{z} M(1)$,

$$
\left\|\left(D \bar{f}_{z}\right)_{v}-\left(D \bar{f}_{z}\right)_{v^{\prime}}\right\| \leq C\left|v-v^{\prime}\right|^{\beta},
$$

where $\left(D \bar{f}_{z}\right)_{v}: T_{z} M \rightarrow T_{f z} M$ is the derivative of $\bar{f}_{z}$ at the point $v$. When $v^{\prime}=0,\left(D f_{z}\right)_{v^{\prime}}=T_{z} f$ since $T_{z} \exp$ is the identity. Define $\varepsilon^{\prime} \leq \varepsilon$ by $1+\varepsilon^{\prime}=$ $(1+\varepsilon)^{\beta}$. Replace $\varepsilon$ by $\varepsilon^{\prime}$ in 3.3 and let $B_{\varepsilon^{\prime}}$ be the corresponding $\varepsilon^{\prime}$-slowly varying function comparing the adapted Finsler to the smooth one over $\mathscr{H}(\tau)$. Set

$$
r_{\varepsilon}(z)=\left(\nu / 2 C B_{\varepsilon^{\prime}}(f z)\right)^{1 / \beta} .
$$

It is $\varepsilon$-slowly varying by choice of $\varepsilon^{\prime}$. If $v \in T_{z} M$ and $|v|^{*} \leq r_{\varepsilon}(z)$ then

$$
\begin{aligned}
\left\|\left(D \bar{f}_{z}\right)_{v}-T_{z} f\right\|^{*} & =\sup _{w \neq 0} \frac{\left|\left(D \bar{f}_{z}\right)_{v}(w)-T_{z} f(w)\right|^{*}}{|w|^{*}} \\
& \leq \sqrt{2} B_{\varepsilon}(f z)\left\|\left(D \bar{f}_{z}\right)_{v}-T_{z} f\right\| \leq 2 B_{\varepsilon}(f z) C|v|^{* \beta} \leq \nu,
\end{aligned}
$$

by choice of $r_{\varepsilon}$. Q.E.D.

We extend 3.4 as follows. Let $\varepsilon, \nu, \beta, \beta^{\prime}>0$ be given with $\beta^{\prime}<\beta$. As always, $f$ is $C^{1+\beta}$. Define $\varepsilon^{\prime \prime}$ by $1+\varepsilon^{\prime \prime}=(1+\varepsilon)^{\beta-\beta^{\prime}}$. Thus, $0<\varepsilon^{\prime \prime}<$ $\varepsilon^{\prime} \leq \varepsilon$. Let $B_{\varepsilon^{\prime \prime}}$ be the Finsler comparison function supplied by 3.3. Define $r: \mathscr{H}(\tau) \rightarrow(0,1)$ by

$$
r(z)=\frac{1}{2}\left(\nu / 2 C B_{e^{\prime \prime}}(f z)\right)^{1 /\left(\beta-\beta^{\prime}\right)} .
$$

Then $r$ is $\varepsilon$-slowly varying and, for $v, v^{\prime} \in T_{z} M$ with $|v|^{*},\left|v^{\prime}\right|^{*} \leq r(z)$,

$$
\begin{aligned}
\left\|\left(D \bar{f}_{z}\right)_{v}-\left(D \bar{f}_{z}\right)_{v^{\prime}}\right\|^{*} & \leq 2 B_{\varepsilon^{\prime \prime}}(f z) C\left|v-v^{\prime}\right|^{* \beta} \\
& \leq 2 B_{\varepsilon^{\prime \prime}}(f z) C(2 r(z))^{\beta-\beta^{\prime}}\left|v-v^{\prime}\right|^{* \beta^{\prime}} \leq \nu\left|v-v^{\prime}\right|^{* \beta^{\prime}} .
\end{aligned}
$$

To sum up, we have proved

3.5. Corollary: $C^{1+\beta^{\prime}}$ uniformity of $\bar{f}$. Given $\varepsilon, \nu, \beta, \beta^{\prime}>0$ with $\beta^{\prime}<\beta$ and $f \in C^{1+\beta}$, there exists an $\varepsilon$-slowly varying Borel radius function $r: \mathscr{H}(\tau) \rightarrow$ $(0,1)$ such that

$$
\left\|\left(D \bar{f}_{z}\right)_{v}-\left(D \bar{f}_{z}\right)_{v^{\prime}}\right\|^{*} \leq \nu\left|v-v^{\prime}\right|^{* \beta^{\prime}}, \quad|v|^{*},\left|v^{\prime}\right|^{*} \leq r(z) .
$$

The function $r^{-1}$ is comparable to a growth control function for Tf on $\mathscr{H}(\tau)$.

Definition. If a growth control function $G_{\varepsilon}$ is bounded on a subset $P$ of $\mathscr{H}(\tau)$ then $P$ is called a hyperbolic block for $G_{\varepsilon}$. Since $P$ need not be $f$-invariant we are led to consider its saturate, $\bigcup_{k \in \mathbf{Z}} f^{k} P$. 
By discarding overlaps and accepting arbitrarily large bounds, we can find a countable family $\mathscr{P}=\{P\}$ of disjoint, Borel, hyperbolic blocks whose saturates partition $\mathscr{H}(\tau)$. For each $P \in \mathscr{P}$ there is a radius function $r_{P}$ given by 3.5 such that $\left\|\left(D \bar{f}_{z}\right)_{v}-\left(D \bar{f}_{z}\right)_{v^{\prime}}\right\|^{*} \leq \nu\left|v-v^{\prime}\right|^{* \beta^{\prime}}$ whenever $|v|^{*},\left|v^{\prime}\right|^{*} \leq r_{P}(z)$, $z \in \operatorname{Sat}(P)$. On $P, r_{P}^{-1}$ is bounded because it is comparable to $G_{\varepsilon}$. Redefine a Borel radius function $r: \mathscr{H}(\tau) \rightarrow(0,1)$ as follows. Each $z \in \mathscr{H}(\tau)$ belongs to $f^{k}(P)$ for some $P \in \mathscr{P}$ and some smallest $|k| ; P$ and $|k|$ are unique. Set

$$
r(z)=(1+\varepsilon)^{-|k|} \inf \left\{r_{P}(p): p \in P\right\} .
$$

Similarly, define $G(z)=(1+\varepsilon)^{|k|} \sup \left\{G_{\varepsilon}(p): p \in P\right\}$. Then $r$ and $G$ are Borel, $\varepsilon$-slowly varying, and constant on $P$. Thus,

3.6. Proposition: Hyperbolic blocks. Given $\varepsilon, \nu, \beta, \beta^{\prime}, f$ as above, there exist an $\varepsilon$-slowly varying growth control function $G$, a countable family $\mathscr{P}$ of hyperbolic blocks for $G$, and a positive, Borel radius function $r: \mathscr{H}(\tau) \rightarrow(0,1)$ such that

(a) The saturates of $P \in \mathscr{P}$ partition $\mathscr{H}(\tau)$.

(b) On each $P, G$ and $r$ are constant.

(c) If $|v|^{*},\left|v^{\prime}\right|^{*} \leq r(z), z \in \operatorname{Sat}(P)$, then $\left\|\left(D \bar{f}_{z}\right)_{v}-\left(D \bar{f}_{z}\right)_{v^{\prime}}\right\|^{*} \leq \nu\left|v-v^{\prime}\right|^{* \beta^{\prime}}$.

Here is another property of hyperbolic blocks.

3.7. Continuity Lemma. The weak hyperbolic splitting of TM over the hyperbolic block $P$ extends to a unique continuous $T f$-invariant splitting $T_{P} M=$ $E^{u} \oplus \bar{E}^{s}$ where $\bar{P}$ is the closure of $P$ in $M$. The growth controls along orbits through $P$ extend to growth controls along orbits through $\bar{P}$.

Proof. Note that $\mathscr{H}$ and $\mathscr{R}$ may fail to contain $P$. Let $p_{k} \rightarrow p$ as $k \rightarrow \infty$, where $p_{k} \in P$ and $p \in \bar{P}$. Since the Grassmannian is compact, we may assume that $E_{p_{k}}^{u}$ and $E_{p_{k}}^{s}$ converge, say to $F^{u}$ and $F^{s}$. By 3.2

$$
\begin{aligned}
& G^{-1} a^{n} \leq\left|T f^{n}(v)\right| \leq G b^{n}, \quad n \geq 0, \\
& G^{-1} b^{n} \leq\left|T f^{n}(v)\right| \leq G a^{n}, \quad n \leq 0,
\end{aligned}
$$

for all unit vectors $\left.v \in E^{u}\right|_{P}, G$ being the value of $G(p)$ on $P$. Since $T f^{n}$ is continuous, the same expansion estimates hold on $F^{u}$. Corresponding contraction estimates hold on $F^{s}$. Now suppose that a second sequence $p_{k}^{\prime}$ tends to $p$ and the subspaces $E_{p_{k}^{\prime}}^{u}, E_{p_{k}^{\prime}}^{s}$ converge, say to $F^{u^{\prime}}$ and $F^{s^{\prime}}$. The expansion and contraction estimates hold on $F^{u^{\prime}}$ and $F^{s^{\prime}}$ too. If $v$ is a vector in $T_{p} M$ which does not lie in $F^{s}$ then $v=x+y$ where $x \in F^{u}, y \in F^{s}$, and $x \neq 0$. As $n \rightarrow \infty,\left|T f^{n}(x)\right|$ grows more rapidly than $a^{n} / G$ while $\left|T f^{n}(y)\right| \rightarrow 0$. Thus, such a $v$ cannot lie in $F^{s^{\prime}}$; i.e., $F^{s^{\prime}} \subset F^{s}$. Similarly, $F^{s} \subset F^{s^{\prime}}$, so they are equal. Likewise, $F^{u}=F^{u^{\prime}}$. It follows that $F^{u} \oplus F^{s}$ is continuous over $\bar{P}$. Saturating the splitting by $T f$-iteration produces a well-defined $T f$-invariant 
splitting over $\operatorname{Sat}(\bar{P})$ because $E^{u} \oplus E^{s}$ is $T f$-invariant, and the growth controls persist. Q.E.D.

It is now an easy matter to find the unstable manifolds through orbits of a hyperbolic block $P \subset \mathscr{H}(\tau)$. We merely work in the adapted Finsler, where $f$ appears to be uniformly hyperbolic, and apply the uniform Unstable Manifold Theorem.

3.8. Pesin's $C^{1}$ Unstable Manifold Theorem on Sat $(P)$. Suppose that the diffeomorphism $f: M \supset$ is of class $C^{1+\beta}, 0<\beta \leq \mathrm{Lip}$, and $r$ is the radius function supplied by 3.6. For each $z \in \operatorname{Sat}(P)$ the unstable set $W^{u}(z)$ is locally the graph of a $C^{1}$ map $w^{u}: E_{z}^{u}(r(z)) \rightarrow E_{z}^{s}(r(z))$, exponentiated into $M$, and $p \mapsto W^{u}(p, \cdot)$ is $C^{1}$-continuous respecting $p \in \bar{P}$. We call this local unstable manifold $W_{z}^{u}(r)$. At $z$ it is tangent to $E_{z}^{u}$. Under $f, \mathscr{W}^{u}(r)=\left\{W_{z}^{u}(r)\right\}$ overflows in the sense that $f\left(W_{z}^{u}(r)\right) \supset W_{f z}^{u}(r)$.

Proof. By 3.7 the weak hyperbolic splitting over $P$ extends to a continuous splitting $\bar{E}^{u} \oplus \bar{E}^{s}$ over the closure $\bar{P}$ of $P$ in $M$. The growth control and adapted Finsler also extend continuously. Let $H$ be the disjoint union of the iterates of $P$, say $H=\sqcup P_{k}$, equipped with the metric

$$
d\left(z, z^{\prime}\right)= \begin{cases}d_{M}\left(z, z^{\prime}\right) & \text { if } z, z^{\prime} \in \bar{P}_{k} \text { for some } k, \\ \operatorname{diam}(M) & \text { if } z \in \bar{P}_{k}, z^{\prime} \in \bar{P}_{k^{\prime}} \text { for some } k \neq k^{\prime} .\end{cases}
$$

By definition, $\bar{P}_{k} \cap \bar{P}_{k^{\prime}}=\varnothing$ for all $k \neq k^{\prime}$ even though $f^{k} \bar{P} \cap f^{k^{\prime}} \bar{P}$ may be nonempty. The restrictions of $\bar{E}^{u}, \bar{E}^{s}$, and $\|\left.\right|^{*}$ to $\bar{P}_{k}$ are continuous. Rescale ||$^{*}$ on $T_{H} M=\sqcup T_{P_{k}} M$ as follows. If $v \in T_{z} M, z \in H$, set $|v|^{* *}=|v|^{*} / r(z)$. Impose ||$^{* *}$ on $T_{H} M$. (Note that ||$^{* *}$ depends on $\varepsilon$ (through $r$ ) but ||$^{*}$ does not.) Respecting ||$^{* *}, \bar{f}_{z}$ is a $C^{1}$-uniformly hyperbolic embedding of the unit ball $U_{z}$. For

$$
\begin{aligned}
\left\|\left(D \bar{f}_{z}\right)_{v}-T_{z} f\right\|^{* *} & =\sup _{w \neq 0} \frac{\left|\left(D \bar{f}_{z}\right)_{v}(w)-T_{z} f(w)\right|^{* *}}{|w|^{* *}} \\
& \leq\left\|\left(D \bar{f}_{z}\right)_{v}-T_{z} f\right\|^{*} \frac{r(z)}{r(f z)} \leq \nu(1+\varepsilon)
\end{aligned}
$$

shows that $\bar{f}_{z}$ is $C^{1}$-uniformly approximated by $T_{z} f$ on $U_{z}$; and $T_{z} f$ is uniformly hyperbolic because, respecting ||$^{* *}$, it expands $E^{u}$ at least as sharply as $\mu /(1+\varepsilon)$ and contracts $E^{s}$ at least as sharply as $(1+\varepsilon) / \mu$. The uniformity refers to $z$ varying over $H$.

The standard graph transform construction of unstable manifolds in [HPS] or [S] applies to the collection of embeddings $\bar{f}_{z}: U_{z} \rightarrow T_{f_{z}} M, z \in H$. In the standard case one requires that $\bar{f}$ lifts a diffeomorphism at a compact hyperbolic set. Although $H$ is not compact, the lift $\bar{f}$ is uniformly hyperbolic, respecting ||$^{* *}$, and that, not compactness, is what counts.

Here are a few details. Let $U_{z}^{u}, U_{z}^{s}$ be the unit balls in $E_{z}^{u}, E_{z}^{s}$ respecting ||$^{* *}$. Choose a family of $C^{1}$ functions $w=\left\{w_{z}\right\}_{z \in H}$ such that $w_{z}: U_{z}^{u} \rightarrow U_{z}^{s}$ 
has $\left\|\left(D w_{z}\right)_{x}\right\|^{* *} \leq 1$ for all $z \in H, x \in U_{z}^{u}$. We may choose the $w_{z}$ to depend continuously on $z \in H$ in the $C^{1}$ sense. (For example, since $E^{u}$ and $E^{s}$ are continuous on $H, w_{z} \equiv 0$ is $C^{1}$ continuous.) The graph of $w_{z}$ is a trial disc $D_{z}$; it is a candidate to be $W_{z}^{u}$. Apply $\bar{f}$ to all the trial discs and cut the images down to unit size: $D_{1, f z}=\tilde{f}\left(D_{z}\right) \cap U_{f z}$. These $D_{1, f z}$ are new trial discs. Let $\mathscr{D}=\left\{D_{z}\right\}_{z \in H}$ and $\mathscr{D}_{1}=\left\{D_{1, z}\right\}_{z \in H}$. By exactly the same estimates as in [HPS, pp. 40-46; S, pp. 39-44], $D_{1, f z}$ is the graph of a $C^{1}$ map $\bar{f}_{\#} w_{z}: U_{f z}^{u} \rightarrow U_{f z}^{s}$ with $\left\|\left(D \bar{f}_{\#} w_{z}\right)_{x}\right\|^{* *} \leq 1$ for all $x \in U_{f z}^{u}$. We have merely graph transformed each and every $w_{z}$ by $\bar{f}_{z}$. Therefore, we can iterate, getting a sequence of families of discs $\mathscr{D}_{n}, n=0,1, \ldots$. Exactly as in [HPS or S] $\mathscr{D}_{n}$ uniformly $C^{1}$-converges as $n \rightarrow \infty$. See 3.9 for a closer look at this convergence.

Note that $C^{1}$-convergence respecting ||$^{* *}$ implies $C^{1}$-convergence respecting $1 \mathrm{I}^{*}$. For when passing from one norm to the other we are merely rescaling by the factor $r(z)$. It is interesting that under such linear rescaling, $C^{r}$-convergence can be lost for $r>1$. In $\S 6$ other means are used to understand the $C^{r}$ nature of the unstable manifolds.

Call $W_{z}^{u}=\lim _{n} D_{n, z}$. This limit disc is $C^{1}$, tangent to $\bar{E}_{z}^{u}$, and overflows in the sense that $\bar{f}\left(\dot{W}_{z}^{u}\right) \supset \bar{W}_{f z}^{u}$. Respecting ||$^{*}, \bar{W}_{z}^{u}$ has size $r(z)$. Call $W_{z}^{u}(r)=\exp \left(W_{z}^{u}\right)$. Then $W_{z}^{u}(r)$ is $C^{1}$, is tangent to $E_{z}^{u}$, and $f\left(W_{z}^{u}(r)\right) \supset$ $W_{f z}^{u}(r)$. As a function of $p \in \bar{P}, W_{p}^{u}(r)$ is $C^{1}$-continuous.

$\bar{f}$ expands $W_{z}^{u}$ by at least the factor $\mu$ and so approximately the same is true of $f$ on $W_{z}^{u}(r)$. For exp has derivative the identity at the zero section and the smooth Riemann structure is comparable to the adapted one by an $\varepsilon$-slowly varying comparison function. Thus, under $f^{-n}$, points of $W_{z}^{u}(r)$ are exponentially asymptotic as $n \rightarrow \infty$. That is, $W_{z}^{u}(r)$ is contained in the unstable set (see $\S 1$ ) of $z, W_{z}^{u}(r) \subset W^{u}(z)$.

On the other hand, suppose that $f^{-n}(q)$ is exponentially asymptotic with $z$ as $n \rightarrow \infty$, say at the rate $\lambda^{n}$ for some $\lambda<1$. For small $\varepsilon$ and large $n$, $\lambda^{n}<r\left(f^{-n} z\right)$ and so $f^{-n}(q)$ lifts to an $\bar{f}$-orbit that is exponentially asymptotic with $z$ inside the neighborhoods $U_{f^{-n} z}$. By the characterization of unstable manifolds in the uniform case, these $\bar{f}^{-n}(\bar{q})$ lie in $\bar{W}_{f^{-n} \bar{q}}^{u}$; that is, locally, $W^{u}(z)$ and $W_{z}^{u}(r)$ coincide. (In particular $W_{z}^{u}(r)$ is uniquely locally determined by $z$ and is independent of $\varepsilon$.) This completes the proof of 3.8. Q.E.D.

Remark 1. The corresponding Stable Manifold Theorem follows, in the diffeomorphism case, by considering $f^{-1}$. For the endomorphism case see $\S 5$.

Remark 2. It is only to get 3.4 that we use $f \in C^{1+\beta}, \beta>0$, in an essential way. If $f$ is merely $C^{1}$ but the conclusion of 3.4 happens to be true anywaythat is, $\bar{f}$ is uniformly $C^{1}$ respecting adapted coordinates on a slowly varying neighborhood of $\mathscr{O}(p)$-then the conclusion of 3.8 holds: $f$ has $C^{1}$ stable and unstable manifolds along $\mathscr{O}(p)$. 
We need to sharpen 3.8 somewhat. We know that the trial discs given by $w_{n}=\bar{f}_{\#}^{n}\left(w_{0}\right)$ converge $C^{1}$-uniformly to $\bar{W}^{u}=\operatorname{graph}\left(w^{u}\right)$. In fact the convergence is exponential in the following sense. Respecting the Finsler ||$^{* *}, \bar{f}$ is $C^{1}$-uniformly hyperbolic with uniform hyperbolicity rate $(1+\varepsilon) / \mu<1$. The proof of the Unstable Manifold Theorem in [HPS or S] produces a contraction at this rate. That is, for some constant $C$,
$\left(a^{* *}\right)$
$\left|w_{n}(x)-w^{u}(x)\right|^{* *} \leq C\{(1+\varepsilon) / \mu\}^{n}$,
$\left(\mathrm{b}^{* *}\right)$

$$
\left\|\left(D w_{n}\right)_{x}-\left(D w^{u}\right)_{x}\right\|^{* *} \leq C\{(1+\varepsilon) / \mu\}^{n} .
$$

(Actually, $C=2$.) These estimates are uniform over all $n \geq 0$, all $x \in E_{z}^{u}(r)$, all $z \in H$, and all choices of the initial family of trial discs $w_{0}$. To sum up,

3.9. Addendum: Exponential $C^{1}$-convergence. Let $H$ be the saturate of a hyperbolic block. If the graphs of $w_{0}: E_{H}^{u}(r) \rightarrow E_{H}^{s}(r)$ form a family of $C^{1}$ trial discs of slope $\leq 1$ with respect to $\left.\right|^{*}$ then the natural iterated graph transform gives a sequence of families of trial discs of slope $\leq 1$ that are graphs of maps $w_{n}: E^{u}(r) \rightarrow E^{s}(r), n \geq 0$, and for all $x \in E_{z}^{u}(r), z \in H$,

$$
\begin{gathered}
\left|w_{n}(x)-w^{u}(x)\right|^{*} \leq C r(z)\{(1+\varepsilon) / \mu\}^{n}, \\
\left\|\left(D w_{n}\right)_{x}-\left(D w^{u}\right)_{x}\right\|^{*} \leq C\{(1+\varepsilon) / \mu\}^{n} .
\end{gathered}
$$

Proof. Since ||$^{*}$ at $z$ is just the dilation of ||$^{* *}$ by $r(z),\|\|^{*}=\|\|^{* *}$ and $\left(\mathrm{a}^{* *}\right),\left(\mathrm{b}^{* *}\right)$ imply $\left(\mathrm{a}^{*}\right),\left(\mathrm{b}^{*}\right)$. Note also that trial discs in $\left(T_{z} M(r),||^{*}\right)$ with slope $\leq 1$ are trial discs in $\left(T_{z} M(1),||^{* *}\right)$ with slope $\leq 1$ and vice versa. Q.E.D.

In the proof that $\mathscr{W}^{u}$ and $\mathscr{W}^{s}$ are absolutely continuous, we will need to understand the Hölder properties of iterated trial discs and the unstable
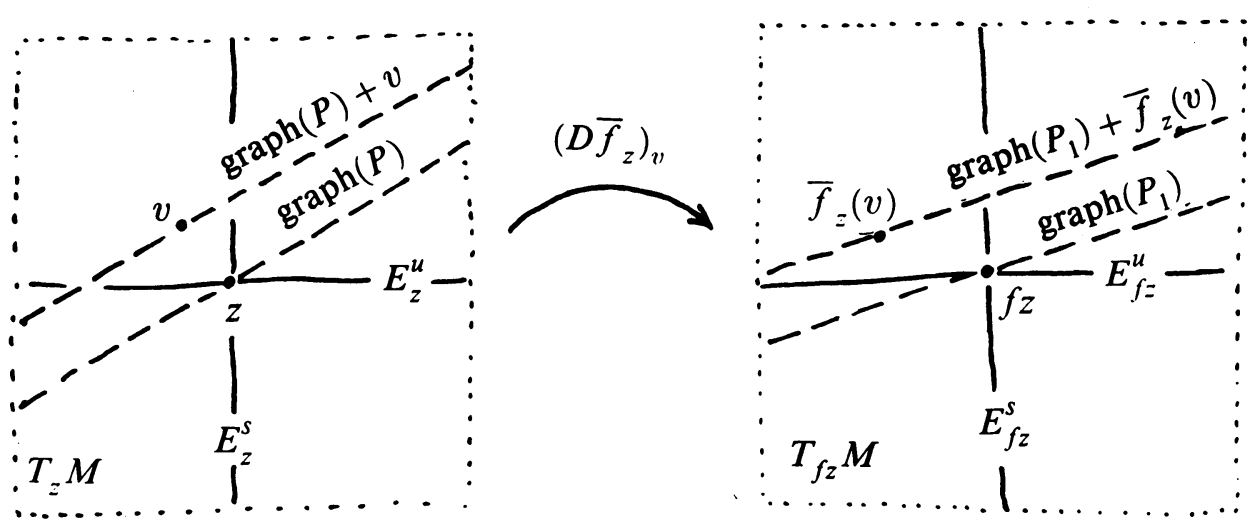

FIgURE 5. The natural action of $D \bar{f}_{z}$ on a prospective tangent plane 
manifold. We take some $v \in T_{z} M(r), z \in H$, and express $\left(D \bar{f}_{z}\right)_{v}$ respecting the $E^{u} \oplus E^{\mathcal{s}}$ splitting as

$$
\left(D \bar{f}_{z}\right)_{v}=\left[\begin{array}{ll}
A_{v} & B_{v} \\
C_{v} & K_{v}
\end{array}\right], \quad \begin{array}{ll}
A_{v}: E_{z}^{u} \rightarrow E_{f z}^{s}, & B_{v}: E_{z}^{s} \rightarrow E_{f z}^{u}, \\
C_{v}: E_{z}^{u} \rightarrow E_{f z}^{s}, & K_{v}: E_{z}^{s} \rightarrow E_{f z}^{s} .
\end{array}
$$

The tangent plane to a trial disc at $v$ can be thought of as the graph of a linear map $P: E_{z}^{u} \rightarrow E_{z}^{s}$. Since the slope of the trial disc is $\leq 1,\|P\|^{*} \leq 1$ also. The natural action of $\left(D \bar{f}_{z}\right)_{v}$ on such planes is $\operatorname{graph}(P) \mapsto \operatorname{graph}\left(P_{1}\right)$, where $\operatorname{graph}\left(P_{1}\right)$ is the $\left(D \bar{f}_{z}\right)_{v}$-image of $\operatorname{graph}(P)$. See Figure 5. A formula describing $P_{1}$ is

$$
P_{1}=T \bar{f}_{\#}(P)=\left(C_{v}+K_{v} P\right) \circ\left(A_{v}+B_{v} P\right)^{-1} .
$$

See [HPS, S], or $\S 6$. By 3.5 and choice of $\mu$, both factors have norm $\leq 1$, so we can iterate, getting $P_{n}: E_{f^{n z}}^{u} \rightarrow E_{f^{n z}}^{s}$ with $\left\|P_{n}\right\|^{*} \leq 1$ and

$$
\operatorname{graph}\left(P_{n}\right)=\left(D \bar{f}_{z}^{n}\right)_{v}(\operatorname{graph}(P)) \text {. }
$$

To apply 3.5 we must assume that $\bar{f}^{i} v \in T M(r), 0 \leq i \leq n$; i.e., $\left|v_{i}\right|^{*} \leq$ $r\left(f^{i} z\right)$. If $v$ lies on $\bar{W}_{z}^{s}(r)$ or is very near it then indeed $\bar{f}^{i} v$ does stay in $T M(r)$. Now suppose that $P^{\prime}: E_{z}^{u} \rightarrow E_{z}^{s}$ represents a second prospective tangent plane. We compare $P_{n}$ and $P_{n}^{\prime}$ where $\operatorname{graph}\left(P_{n}^{\prime}\right)=\left(D \bar{f}_{z}^{n}\right)_{v^{\prime}}\left(\operatorname{graph}\left(P^{\prime}\right)\right)$, $v^{\prime}$ being some other point of $T_{z} M(r)$. We call $v_{i}^{\prime}=\bar{f}^{i}\left(v_{i}^{\prime}\right)$ and we assume that $v_{i}^{\prime}$ also lies in $T_{f^{i} z} M(r), 0 \leq i \leq n$.

\subsection{Proposition.}

$$
\left\|P_{n}-P_{n}^{\prime}\right\|^{*} \leq C \sum_{1 \leq i \leq n} \mu^{-i}\left|v_{n-i}-v_{n-i}^{\prime}\right|^{* \beta^{\prime}}+\mu^{-n}\left\|P-P^{\prime}\right\|^{*}
$$

where $C$ is a uniform constant, $0<\beta^{\prime}<\beta$, and, as above, $\mu$ under-bounds the hyperbolicity of $T f$ respecting the adapted Finsler.

Proof. Omit the subscripts " $v$ " and write $\left\|P_{1}-P_{1}^{\prime}\right\|^{*}$ as

$$
\begin{aligned}
\leq & \left\|(C+K P)-\left(C^{\prime}+K^{\prime} P^{\prime}\right)\right\|^{*}\left\|(A+B P)^{-1}\right\|^{*} \\
& +\left\|C^{\prime}+K^{\prime} P^{\prime}\right\|^{*} \|\left(A^{\prime}+B^{\prime} P^{\prime}\right)^{-1}\left(A^{\prime}+B^{\prime} P^{\prime}\right)(A+B P)^{-1} \\
& -\left(A^{\prime}+B^{\prime} P^{\prime}\right)^{-1}(A+B P)(A+B P)^{-1} \|^{*} \\
\leq & \left\{\left\|C-C^{\prime}\right\|^{*}+\left\|K\left(P-P^{\prime}\right)\right\|^{*}+\left\|\left(K-K^{\prime}\right) P\right\|^{*}\right\} \mu^{-1} \\
& +\mu^{-1}\left\{\mu^{-1}\left[\left\|A-A^{\prime}\right\|^{*}+\left\|B^{\prime}\left(P^{\prime}-P\right)\right\|^{*}+\left\|\left(B^{\prime}-B\right) P\right\|^{*}\right] \mu^{-1}\right\} \\
\leq & 4 \nu\left|v-v^{\prime}\right|^{* \beta^{\prime}}+\mu^{-1}\left\|P-P^{\prime}\right\|^{*},
\end{aligned}
$$

where $\nu$ is the $\beta^{\prime}$-Hölder constant of $T \bar{f}$ respecting ||$^{*}, 0<\beta^{\prime}<\beta$. See 3.5. Beginning with $\left\|P_{n}-P_{n}^{\prime}\right\|^{*}$ and applying this inequality repeatedly gives the result. Q.E.D.

3.11. Corollary: $C^{1+\beta^{\prime}}$-uniformity of $W_{z}^{u}$. Over the saturate $H$ of a hyperbolic block the discs $\bar{W}_{z}^{u} \in \mathscr{W}^{u}$ are uniformly of class $C^{1+\beta^{\prime}}, \beta^{\prime}<\beta$, and the same is 
true of $\bar{f}^{n}$-iterated families of trial discs converging to $\mathscr{W}^{u}$. In particular, each $W_{z}^{u}(r)$ is of class $C^{1+\beta^{\prime}}$. Everything is measured with respect to the adapted Finsler.

Proof. As usual, the diffeomorphism $f$ is assumed to be $C^{1+\beta}, \beta>0$. Let $w_{z}: E_{z}^{u}(r) \rightarrow E_{z}^{s}(r)$ be a family of $C^{1+\beta^{\prime}}$ maps such that $\left\|D w_{z}\right\|^{*} \leq 1$ and the $\beta^{\prime}$-Hölder constant of $D w_{z}$ is uniformly bounded as $z$ ranges over $H$. For instance, we could take $w_{z} \equiv 0$. Let $\mathscr{D}$ denote the family of trial discs which are the graphs of the maps $w_{z}$ and let $\mathscr{D}_{n}$ denote the $\bar{f}^{n}$-iteration of $\mathscr{D}$. The disc $D_{n z} \in \mathscr{D}_{n}$ is $\bar{f}^{n}\left(D_{f^{-n} z}\right)$, cut down to size $r(z)$. Let $w_{n z}$ be the map whose graph is $D_{n z}$. Let $P_{n}, P_{n}^{\prime}$ represent the tangent planes at some $v_{n}, v_{n}^{\prime} \in D_{n z}$. Automatically, $\bar{f}^{-j}\left(v_{n}\right)=v_{j}$ and $\bar{f}^{-j}\left(v_{n}^{\prime}\right)=v_{j}^{\prime}$ lie in $T M(r)$, $0 \leq j \leq n$. By 3.10 ,

$$
\left\|P_{n}-P_{n}^{\prime}\right\|^{*} \leq C \sum_{1 \leq i \leq n} \mu^{-i}\left|v_{n-i}-v_{n-i}^{\prime}\right|^{* \beta^{\prime}}+\mu^{-n}\left\|P-P^{\prime}\right\|^{*}
$$

where $P, P^{\prime}$ are the tangent planes to $D_{f^{-n_{z}}}$ at $v=f^{-n}\left(v_{n}\right), v^{\prime}=f^{-n}\left(v_{n}^{\prime}\right)$. Since $\bar{f}$ expands horizontally more sharply than $\mu$, we see that $\left|v_{n-i}-v_{n-i}^{\prime}\right|^{*} \leq$ $\mu^{-i}\left|v_{n}-v_{n}^{\prime}\right|^{*}$. Since $D_{f^{-n z}}$ is $C^{1+\beta^{\prime}},\left\|P-P^{\prime}\right\|^{*} \leq L\left|v-v^{\prime}\right|^{* \beta^{\prime}} \leq L\left|v_{n}-v_{n}^{\prime}\right|^{* \beta^{\prime}}$. Thus,

$$
\left\|P_{n}-P_{n}^{\prime}\right\|^{*} \leq\left\{C \sum_{i \geq 1} \mu^{-i}+L\right\}\left|v_{n}-v_{n}^{\prime}\right|^{* \beta^{\prime}} .
$$

It follows that $w_{n z}$ has uniformly bounded $C^{1+\beta^{\prime}}$ size. The $\beta^{\prime}$-Hölder constant of $D w_{n z}$ is $\leq\{C /(\mu-1)+L\}$. By $3.8 w_{n}$ converges $C^{1}$-uniformly to $w^{u}$. Therefore $w^{u}$ is also uniformly $C^{1+\beta^{\prime}}$. Thus, $\bar{W}_{z}^{u}$ and $W_{z}^{u}$ are $C^{1+\beta^{\prime}}$ too. Q.E.D.

Remark. Actually, $W_{z}^{u}$ is of class $C^{1+\beta}$. See Theorem 6.4.

Theorem 3.8 describes $\bar{W}^{u}$ locally in the adapted norm $\mid{ }^{*}$, but we can also ask how it looks in the original smooth norm ||. The space $T_{z} M$ is split in two ways:

$$
E_{z}^{u} \oplus E_{z}^{s}=T_{z} M=E_{z}^{u} \oplus E_{z}^{u \perp}
$$

where $E^{u \perp}$ denotes the orthogonal complement of $E^{u}$ respecting the smooth Riemann structure $\langle$,$\rangle . We will find subdiscs of \bar{W}_{z}^{u}$ which are graphs of maps $g_{z}: E_{z}^{u}\left(r^{\prime}\right) \rightarrow E_{z}^{u \perp}\left(r^{\prime}\right)$ where $r^{\prime}$ is comparable to $r$ and the $C^{1+\beta^{\prime}}$ size of $g_{z}$ is uniformly bounded. We call them round u-discs of radius $r^{\prime}$. This is an improvement to 3.8. Although the adapted norm is needed to get the local unstable manifolds to be overflowing, the smooth norm can be used to view them as graphs. The same will be done for iterated trial discs converging to $W^{u}$. This is a more delicate issue than just changing coordinates. We need to use the Hölder properties of the unstable manifolds. See Theorem 3.13. 
It is easier to abstract the situation a little. Let $E$ be a Banach space with two equivalent norms and two splittings. Say the norms are || and ||$^{\prime}$, the splittings are $E_{1} \oplus E_{2}=E=E_{1}^{\prime} \oplus E_{2}^{\prime}$, and $\pi_{i}: E \rightarrow E_{i}, \pi_{i}^{\prime}: E \rightarrow E_{i}^{\prime}$ are the corresponding projections, $i=1,2$. We assume

$$
\left\|\pi_{1}\right\|=1=\left\|\pi_{2}\right\|, \quad\left\|\pi_{1}^{\prime}\right\|^{\prime}=1=\left\|\pi_{2}^{\prime}\right\|^{\prime}
$$

where \|\| and \|\|$^{\prime}$ are the operator norms induced by || and ||$^{\prime}$. Note that (1) holds if $\langle\rangle,,\langle,\rangle^{\prime}$ induce ||$,||^{\prime}$ and $E_{1} \perp E_{2}, E_{1}^{\prime} \perp E_{2}^{\prime}$. Let $B$ be a comparison constant for the norms, $B^{-1} \leq\|\mid /\|^{\prime} \leq B$. Note that \|\|$^{\prime} \leq B^{2}\|\|$ and vice versa. Set $E_{i}(r)=\left\{v \in E_{i}:|v| \leq r\right\}$, and $E_{i}^{\prime}(r)=\left\{v \in E_{i}^{\prime}:|v|^{\prime} \leq r\right\}$ for $i=1,2$.

Now suppose that $g$ is a given map $E_{1}\left(r_{1}\right) \rightarrow E_{2}$. Its graph is an $E_{1}$-disc $G$ in $E, G=\left\{x+g(x): x \in E_{1}(r)\right\}$. We give sufficient conditions for there to exist a map $g^{\prime}: E_{1}^{\prime}\left(r_{1}^{\prime}\right) \rightarrow E_{2}^{\prime}$ such that

$$
r_{1}^{\prime}=r_{1} / 2 B \text { and } G \text { contains } G^{\prime}=\operatorname{graph}\left(g^{\prime}\right) \text {. }
$$

3.12. Lemma. Let splittings, norms, and projections be given that satisfy (1). Assume that $E_{1}=E_{1}^{\prime}$, although || and ||$^{\prime}$ may be different norms on $E_{1}$. Suppose that $G$ is the graph of a $C^{1}$ map $g: E_{1}\left(r_{1}\right) \rightarrow E_{2}$ and

$$
|g(0)| \leq \frac{1}{4} r_{1}, \quad B^{2}\|D g\| \leq \frac{1}{4} .
$$

Then there is a unique $C^{1}$ map $g^{\prime}: E_{1}^{\prime}\left(r_{1}^{\prime}\right) \rightarrow E_{2}^{\prime}$ which obeys (2). Also,

$$
\left|g^{\prime}\right|^{\prime} \leq B^{2}|g| \text { and }\left\|D g^{\prime}\right\|^{\prime} \leq B^{2}\|D g\| \text {. }
$$

By $\left|g^{\prime}\right|^{\prime}$, etc., we refer to $\sup _{x}\left|g^{\prime}(x)\right|^{\prime}$, etc.

Proof. Figure 6 shows us what to do. It is clear that $g^{\prime}$ satisfying (2) is unique and $C^{1}$ if it exists. We define

$$
h(x)=x+\pi_{1}^{\prime}(g x), \quad g^{\prime}(x)=\pi_{2}^{\prime}\left(g\left(h^{-1}(x)\right)\right) .
$$

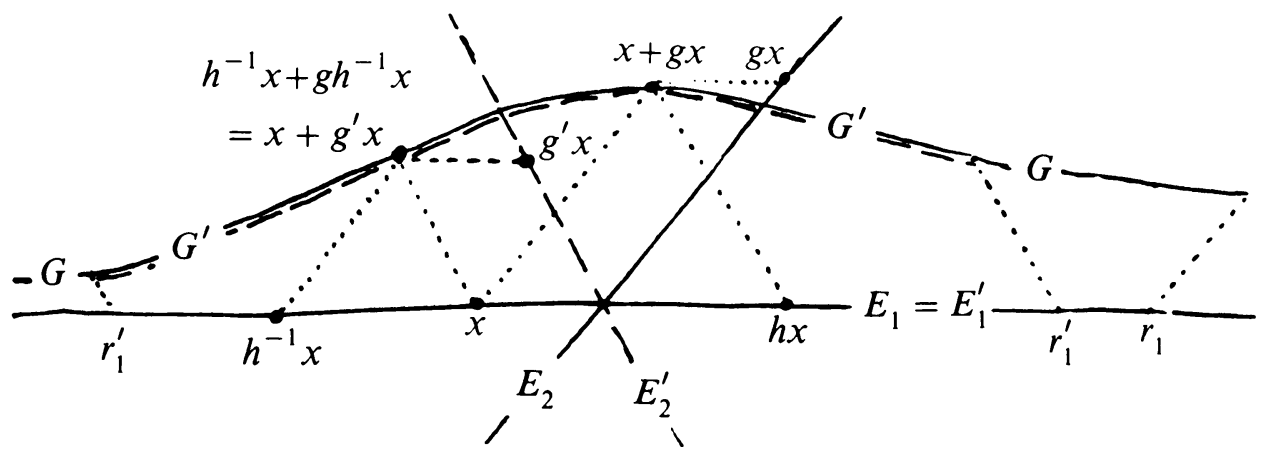

Figure 6. How to view the disc $G$ as a disc $G$ 
By (1) and (3), $\left\|D h-i d_{1}\right\| \leq\left\|\pi_{1}^{\prime}\right\|\|D g\| \leq B^{2}\|D g\| \leq \frac{1}{4}$, so by the Inverse Function Theorem, we see that $h$ embeds $E_{1}\left(r_{1}\right)$ onto a set containing $E_{1}\left(\frac{1}{2} r_{1}\right)$. Besides, $\operatorname{Lip}\left(h^{-1}\right) \leq 2$. Since $|v|^{\prime} \leq r_{1}^{\prime}=r_{1} / 2 B$ implies $|v| \leq B|v|^{\prime} \leq \frac{1}{2} r_{1}$, we see that $h^{-1}$ is well defined on $E_{1}^{\prime}\left(r_{1}^{\prime}\right)$. This makes $g^{\prime}$ well defined and (2) is satisfied by construction. Property (4) is immediate. Q.E.D.

3.13. Proposition: Round and flat $u$-discs. In the sense explained below, unstable manifolds and iterated trial discs contain round, fairly flat u-discs of comparable radius.

Given the $C^{1+\beta}$ diffeomorphism $f$ of $M$ and given $\varepsilon>0$, we find $\varepsilon$ slowly varying Borel radius functions $r, r^{\prime}: \mathscr{H} \rightarrow(0,1)$ and a countable family $\mathscr{P}$ of hyperbolic blocks $P$ whose saturates partition $\mathscr{H}$ such that $r, r^{\prime}$ are constant on each $P$ and $W_{z}^{u}(r)$ contains a round $u$-disc of radius $r^{\prime}(z)$ for all $z \in \operatorname{Sat}(P)$. The maps $g_{z}: E_{z}^{u}\left(r^{\prime}\right) \rightarrow E_{z}^{u \perp}$ whose graphs are these round $u$-discs in $\bar{W}_{z}^{u}$ are of class $C^{1+\beta^{\prime}}, 0<\beta^{\prime}<\beta$, and if $z \in f^{k} P$ then they are fairly flat in the sense that $\left|g_{z}\right|_{C^{1}} \rightrightarrows 0$ as $|k| \rightarrow \infty$. The radius $r^{\prime}(z)$ of the disc on which $g_{z}$ is defined varies slowly as $k \rightarrow \infty$.

If $D=\operatorname{graph}(w)$ is a trial disc in $T_{p} M(r), p \in P$, and $D_{n}=\operatorname{graph}\left(w_{n}\right)$ is its $\bar{f}^{n}$-image, cut down to size $r\left(f^{n} p\right)$, then, for large $n, D_{n}$ contains a round $u$-disc $G_{n}$ of radius $r^{\prime}\left(f^{n} p\right)$, and it too is fairly flat $-\left|g_{n}\right|_{C^{\prime}} \rightrightarrows 0$, as $n \rightarrow \infty$. Everything is measured with respect to the unadapted Finsler $\|$. This family $\mathscr{P}$ is probably finer than the one produced in 3.6 for an $\varepsilon$-slowly varying growth control function.

Proof. It suffices to consider the set $\mathscr{H}(\tau)$ of weak hyperbolic orbits whose growth rates lie outside $\left[\tau^{-1}, \tau\right]$. As in 3.3 , we choose the constant $\mu, 1<$ $\mu<\tau$ and construct the adapted Finsler ||$^{*}$. We may assume $1+\varepsilon<\mu$. Choose any $\beta^{\prime}, \beta^{\prime \prime}, 0<\beta^{\prime}<\beta^{\prime \prime}<\beta$. Next, fix $\varepsilon^{\prime \prime}$ and then $\varepsilon^{\prime}, 0<\varepsilon^{\prime}<\varepsilon^{\prime \prime}<\varepsilon$, such that

$$
\left(1+\varepsilon^{\prime}\right)^{2}<\left(1+\varepsilon^{\prime \prime}\right)^{\beta^{\prime \prime}}<\left(1+\varepsilon^{\prime \prime}\right)^{3}<1+\varepsilon<\mu .
$$

By 3.2, 3.6, there exist a $\frac{1}{3} \varepsilon^{\prime}$-slowly varying Borel growth control function $G$, an $\varepsilon^{\prime}$-slowly varying Borel radius function $r: \mathscr{H}(\tau) \rightarrow(0,1)$, and a countable family $\mathscr{P}$ of hyperbolic blocks $P$ on which $G, r$ are constant. Fix a $P \in \mathscr{P}$ and let $H=\sqcup P_{k}$ where the $P_{k}=f^{k} P, k \in \mathbb{Z}$, as in 3.8. By 3.8, 3.11 the local unstable manifolds of radius $r$ exist and are uniformly $C^{1+\beta^{\prime \prime}}$ over $H$, everything being measured with respect to ||$^{*}$. That is, $\bar{W}_{z}^{u}$ is the graph of a $C^{1+\beta^{\prime \prime}} \operatorname{map} w_{z}^{u}: E_{z}^{u}(r) \rightarrow E_{z}^{s}(r)$ such that $w_{z}^{u}(0)=0,\left(D w_{z}^{u}\right)_{0}=0$, and $D w_{z}^{u}$ is uniformly $\beta^{\prime \prime}$-Hölder. Let $\nu \geq 1$ be its $\beta^{\prime \prime}$-Hölder constant.

Let $B_{\varepsilon^{\prime}}$ be an $\varepsilon^{\prime}$-slowly varying Borel comparison function for the Finslers,

$$
B_{\varepsilon^{\prime}}(z)^{-1} \leq|v|^{*} /|v| \leq B_{\varepsilon^{\prime}}(z), \quad 0 \neq v \in T_{z} M, z \in H .
$$


Choose a constant $c>0$ such that for all $p \in P$

$$
4 B_{\varepsilon^{\prime}}(p)^{2} \nu c^{\beta^{\prime \prime}-\beta^{\prime}} \leq 1
$$

(6) is satisfiable because, by $3.3, B_{\varepsilon^{\prime}}$ is bounded where $G$ is bounded and $G$ is constant on $P$ by construction. (The same cannot be said for a hyperbolic block on which an $\varepsilon$-slowly varying growth control function is constant. We need $\varepsilon^{\prime}$, not $\varepsilon$.) Define $r_{1}: H \rightarrow(0,1)$ by

$$
r_{1}(z)=\operatorname{cr}(P)\left(1+\varepsilon^{\prime \prime}\right)^{-|k|} \quad \text { if } z \in P_{k},
$$

$r(P)$ being the constant value of $r$ on $P$. Since $r$ is $\varepsilon^{\prime}$-slowly varying, $r_{1}<r$. Clearly $r_{1}$ is $\varepsilon^{\prime \prime}$-slowly varying. Call $w_{0}$ the restriction of $w_{z}^{u}$ to $E_{z}^{u}\left(r_{1}\right)$, $z \in H$. We want to apply 3.12 to $w_{0}$ where $B=B_{\varepsilon^{\prime}}(z)$, the two splittings are $E_{z}^{u} \oplus E_{z}^{s}=T_{z} M=E_{z}^{u} \oplus E_{z}^{u \perp}$, and the two norms are ||$^{*},||$. It is clear that condition (1) is met. For (3) we must check two things: $\left|w_{0}(0)\right|^{*} \leq \frac{1}{4} r_{1}$ and $B^{2}\left\|D w_{0}\right\|^{*} \leq \frac{1}{4}$. Since $w_{0}(0)=0$, the first is trivial. Also, $D w_{0}$ is uniformly $\beta^{\prime \prime}$-Hölder, $|x|^{*} \leq r_{1}$, and $\left(D w_{0}\right)_{0}=0$, so we have

$$
B^{2}\left\|\left(D w_{0}\right)_{x}\right\|^{*} \leq B^{2} \nu|x|^{* \beta^{\prime \prime}} \leq\left(B_{\varepsilon^{\prime}}(p)\right)^{2} \nu r(P) c^{\beta^{\prime \prime}}\left(\frac{\left(1+\varepsilon^{\prime}\right)^{2}}{\left(1+\varepsilon^{\prime \prime}\right)^{\beta^{\prime \prime}}}\right)^{|k|} .
$$

By (5), (6) we see that $B^{2}\left\|\left(D w_{0}\right)_{x}\right\|^{*} \leq \frac{1}{4}$ for all $k \in \mathbb{Z}$, and

$$
B^{2}\left\|\left(D w_{0}\right)_{x}\right\|^{*} \rightrightarrows 0 \text { as }|k| \rightarrow \infty \text {. }
$$

From 3.12 we conclude that $\bar{W}_{z}^{u}$ contains a round disc $G_{z}=\operatorname{graph}\left(g_{z}\right)$ of radius $r^{\prime}(z)=r_{1}(z) / 2 B_{\varepsilon^{\prime}}(z)$ and $\left|g_{z}\right|_{C^{1}} \leq B^{2}\left|w_{0}\right|_{C^{1}}$. By (5), $r^{\prime}$ is $\varepsilon$-slowly varying. Also, for $x \in E_{z}^{u}\left(r_{1}\right), z \in P_{k}$, and $|k| \rightarrow \infty$,

$$
B^{2}\left|w_{0}\right|_{C^{1}} \leq B_{\varepsilon^{\prime}}(z)^{2}\left\{r_{1}(z)+1\right\}\left\|\left(D w_{0}\right)_{x}\right\|^{*} \rightrightarrows 0
$$

by (7). Hence $\left|g_{z}\right|_{C^{1}} \rightrightarrows 0$ as $|k| \rightarrow \infty$.

Now suppose that $D$ is a $C^{1}$ trial disc in $T_{p} M(r)$ and $D_{n}$ is its $\bar{f}^{n}$-image, cut down to size $r_{1}\left(f^{n} p\right), n \geq 0$. To apply 3.12 to $D_{n}$ we check two things: $\left|w_{n}(0)\right|^{*} \leq \frac{1}{4} r_{1}\left(f^{n} p\right)$ and $B^{2}\left\|D w_{n}\right\|^{*} \leq \frac{1}{4}$. By 3.9, (6), (7) we see that

$$
\left|w_{n}(0)\right|^{*} \leq C\left(\frac{1+\varepsilon^{\prime \prime}}{\mu}\right)^{n} r_{1}\left(f^{n} p\right) \leq \frac{1}{4} r_{1}\left(f^{n} p\right) \text { for } n \text { large }
$$

and

$$
\begin{aligned}
& B^{2}\left\|D w_{n}\right\|^{*} \leq B^{2}\left\{\left\|D w_{n}-D w_{0}\right\|^{*}+\left\|D w_{0}\right\|^{*}\right\} \\
& \quad \leq B^{2} C\left(\frac{1+\varepsilon^{\prime \prime}}{\mu}\right)^{n}+B^{2}\left\|D w_{0}\right\|^{*} \leq C B_{\varepsilon^{\prime}}(p)^{2}\left(\frac{\left(1+\varepsilon^{\prime \prime}\right)^{3}}{\mu}\right)^{n}+B^{2}\left\|D w_{0}\right\|^{*},
\end{aligned}
$$

which converges to 0 as $n \rightarrow \infty$. By 3.12, for large $n, D_{n}$ contains a round $u$-disc $G_{n}=\operatorname{graph}\left(g_{n}\right)$ of radius $r^{\prime}\left(f^{n} p\right)$, and just as for $w_{0},\left|g_{n}\right|_{C^{\prime}} \rightrightarrows 0$ as $n \rightarrow \infty$. Q.E.D. 
We also will need to understand the Jacobians of $\bar{f}$ on trial discs. Ordinarily, one only speaks of the determinant of a linear transformation of a vector space into itself, $T: V \supset$. But it is also possible to speak of the determinant of $T: V \rightarrow W$ if $V$ and $W$ are finite dimensional inner product spaces. For any $n$ vectors $w_{1}, w_{2}, \ldots, w_{n} \in W$, let $Q$ be the parallelotope $\left\{\sum_{i} a_{i} w_{i}: 0 \leq\right.$ $\left.a_{i} \leq 1\right\}$. It has a well-defined $n$-dimensional volume $\operatorname{vol}(Q)=\left|w_{1}^{\prime}\right|\left|w_{2}^{\prime}\right| \cdots\left|w_{n}^{\prime}\right|$ where $w_{1}^{\prime}=w_{1} ; w_{2}^{\prime}$ is the component of $w_{2}$ normal to $w_{1} ; \ldots$, and $w_{n}^{\prime}$ is the component of $w_{n}$ normal to $\operatorname{span}\left\{w_{1}, \ldots, w_{n-1}\right\}=\operatorname{span}\left\{w_{1}^{\prime}, \ldots, w_{n-1}^{\prime}\right\}$. $\operatorname{Vol}(Q)$ is independent of the order in which the vectors occur.

The determinant of $T: V \rightarrow W$ is the volume of the parallelotope spanned by $T\left(e_{1}\right), T\left(e_{2}\right), \ldots, T\left(e_{n}\right)$ where $e_{1}, e_{2}, \ldots, e_{n}$ is an orthonormal basis of $V$. It is well defined and satisfies the usual properties of determinants: $\operatorname{det}(T) \neq 0$ iff $T$ is $1-1, \operatorname{det}(S \circ T)=\operatorname{det}(S) \operatorname{det}(T)$. If one wishes to give $\operatorname{det}(T)$ a sign, one must assume $V$ and $W$ are oriented and have the same dimension. We do not need signed determinants below.

Now consider a smooth (or $C^{1+\beta^{\prime}}$ ) trial disc $D$ in $T_{p} M(r)$. As in 3.8, let $D_{i}$ be $\bar{f}^{i} D$, cut down to size $r\left(f^{i} p\right)$, and suppose that for some $v \in$ $D, v_{i}=\bar{f}^{i} v \in T M(r), 0 \leq i \leq n$. The tangent to $\left.\bar{f}\right|_{D_{i}}$ is a linear map $T \bar{f}: T_{v_{i}} D_{i} \rightarrow T_{v_{i+1}} D_{i+1}$ and it has a determinant respecting the adapted inner product $\langle,\rangle^{*}$. We call this determinant the Jacobian of $\left.\bar{f}\right|_{D_{i}}$ at $v_{i}$ and denote it by $J^{*}\left(v_{i}\right)$. Let $D^{\prime}$ be a second trial disc in $T_{p} M(r)$ containing a second such point $v^{\prime}$. Call $q, q^{\prime}$ the points where $D, D^{\prime}$ cross $\bar{W}_{p}^{s}$ and call $q_{i}=\bar{f}^{i} q$, $q_{i}^{\prime}=\bar{f}^{i} q^{\prime}$. See Figure 7. We compare the Jacobians at the various points as follows.

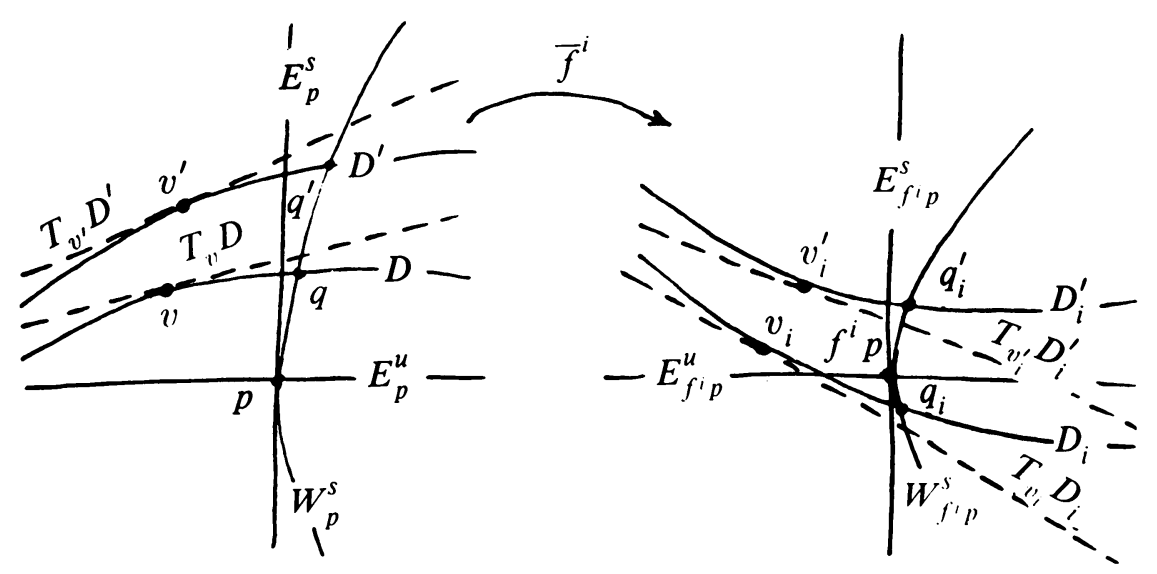

Figure 7. Iterated trial discs 
3.14. Proposition: Jacobians. Over a hyperbolic block, the infinite product $\prod_{i=1}^{\infty} J^{*}\left(q_{i}\right) / J^{*}\left(q_{i}^{\prime}\right)$ converges uniformly and the finite products

$$
\prod_{i=1}^{n} \frac{J^{*}\left(v_{i}\right)}{J^{*}\left(v_{i}^{\prime}\right)}
$$

are uniformly bounded. If $\left|v_{n}-q_{n}\right|^{*}$ and $\left|v_{n}^{\prime}-q_{n}^{\prime}\right|^{*}$ are small and $n$ is large the finite product approximates the infinite one.

Proof. We know that respecting the adapted Finsler, $T \bar{f}$ expands $E^{u}$ by a factor $>\mu$ and contracts $E^{s}$ by a factor $<\mu^{-1}$. Thus, for $0 \leq i \leq n$,

$$
\left|q_{i}-q_{i}^{\prime}\right|^{*} \leq \mu^{-i}\left|q_{0}-q_{0}^{\prime}\right|^{*} \leq \mu^{-i}
$$

Let the graphs of $Q_{n}, Q_{n}^{\prime}: E^{u} \rightarrow E^{s}$ represent $T_{q_{n}}\left(D_{n}\right)$ and $T_{q_{n}^{\prime}}\left(D_{n}^{\prime}\right)$. Then by 3.10 ,

$$
\begin{aligned}
\left\|Q_{n}-Q_{n}^{\prime}\right\|^{*} & \leq C \sum_{1 \leq i \leq n} \mu^{-i}\left|q_{n-i}-q_{n-i}^{\prime}\right|^{* \beta^{\prime}}+\mu^{-n} \\
& \leq C \sum_{1 \leq i \leq n} \mu^{-i}\left(\mu^{-\beta^{\prime}}\right)^{n-i}+\mu^{-n} \\
& \leq C n\left(\mu^{-\beta^{\prime}}\right)^{n}+\mu^{-n}
\end{aligned}
$$

This quantity is exponentially small; i.e.,

$$
\left|q_{n}-q_{n}^{\prime}\right|^{*}+\left\|Q_{n}-Q_{n}^{\prime}\right\|^{*} \leq C^{\prime}\left(\mu^{-\beta^{\prime \prime}}\right)^{n}
$$

for some constant $C^{\prime}$ and some $\beta^{\prime \prime}, 0<\beta^{\prime \prime}<\beta^{\prime}$. Thus,

$$
\left|J^{*}\left(q_{n}\right)-J^{*}\left(q_{n}^{\prime}\right)\right| \leq \nu\left|C^{\prime}\left(\mu^{-\beta^{\prime \prime}}\right)^{n}\right|^{\beta^{\prime}} \leq C^{\prime \prime}\left(\mu^{-\beta^{\prime} \beta^{\prime \prime}}\right)^{n}
$$

By 3.3, $T \bar{f}^{-1}$ is bounded respecting $\mid \|^{*}$, so $J^{*}\left(q_{n}^{\prime}\right)$ is bounded below and

$$
\left|J^{*}\left(q_{n}\right) / J^{*}\left(q_{n}^{\prime}\right)-1\right| \leq C^{\prime \prime \prime}\left(\mu^{-\beta^{\prime \prime \prime}}\right)^{n}
$$

for a constant $C^{\prime \prime \prime}$ and a $\beta^{\prime \prime \prime}, 0<\beta^{\prime \prime \prime}<\beta^{\prime \prime}$. This proves that the infinite product converges.

The estimate on the finite product is similar. It suffices to show that

$$
\prod_{j=1}^{n} \frac{J^{*}\left(v_{j}\right)}{J^{*}\left(q_{j}\right)} \text { and } \prod_{j=1}^{n} \frac{J^{*}\left(v_{j}^{\prime}\right)}{J^{*}\left(q_{j}^{\prime}\right)}
$$

are uniformly bounded as $n \rightarrow \infty$. Since $\bar{f}$ expands $D_{j-i}$ by a factor $>\mu$, we see that $\left|v_{j-i}-q_{j-i}\right|^{*} \leq \mu^{-i}\left|v_{j}-q_{j}\right|^{*}$. Therefore, by 3.10 , and the fact that $D$ is $C^{1+\beta^{\prime}}$,

$$
\begin{aligned}
\left\|P_{j}-Q_{j}\right\|^{*} & \leq C \sum_{1 \leq i \leq j} \mu^{-i}\left|v_{j-i}-q_{j-i}\right|^{* \beta^{\prime}}+\mu^{-j}\left\|P_{0}-Q_{0}\right\|^{*} \\
& \leq C \sum_{1 \leq i \leq j}\left(\mu^{-\beta^{\prime}}\right)^{i}\left|v_{j}-q_{j}\right|^{* \beta^{\prime}}+\mu^{-j} L|v-q|^{* \beta^{\prime}} \\
& \leq C^{\prime}\left|v_{j}-q_{j}\right|^{* \beta^{\prime}}
\end{aligned}
$$


Thus, $\left|v_{j}-q_{j}\right|^{*}+\left\|P_{j}-Q_{j}\right\|^{*} \leq C^{\prime}\left|v_{j}-q_{j}\right|^{* \beta^{\prime}}$, and by 3.5 ,

$$
\begin{aligned}
\left|J^{*}\left(v_{j}\right)-J^{*}\left(q_{j}\right)\right|^{*} & \leq C^{\prime \prime}\left|v_{j}-q_{j}\right|^{* \beta^{\prime \prime}} \leq C^{\prime \prime}\left(\left(\mu^{-1}\right)^{n-j}\right)^{\beta^{\prime \prime}} \\
& =C^{\prime \prime}\left(\mu^{-\beta^{\prime \prime}}\right)^{n-j} .
\end{aligned}
$$

Taking the logarithm of $\prod^{*}\left(v_{j}\right) / J^{*}\left(q_{j}\right)$ gives

$$
\begin{aligned}
\left|\log \prod\right| & \leq \sum_{1 \leq j \leq n}\left|\log \left(J^{*}\left(v_{j}\right)\right)-\log \left(J^{*}\left(q_{j}\right)\right)\right| \\
& \leq \sum_{1 \leq j \leq n} \frac{C^{\prime \prime}\left(\mu^{-\beta^{\prime \prime}}\right)^{n-j}}{\theta_{j}}
\end{aligned}
$$

for some $\theta_{j}$ between $J^{*}\left(v_{j}\right)$ and $J^{*}\left(q_{j}\right)$. But since $T \bar{f}$ and $T \bar{f}^{-1}$ are bounded respecting ||$^{*}$, the $\theta_{j}$ are bounded away from 0 and $\log \Pi$ is dominated by a convergent series $C^{\prime \prime \prime} \sum\left(\mu^{-\beta^{\prime \prime}}\right)^{k}$. Hence, the finite product $\prod J^{*}\left(v_{j}\right) / J^{*}\left(v_{j}^{\prime}\right)$ is uniformly bounded. If $\left|v_{n}-q_{n}\right|^{*}$ and $\left|v_{n}^{\prime}-q_{n}^{\prime}\right|^{*}$ are small while $n$ is large then the same estimates show that the finite product approximates the infinite one. Q.E.D.

Now we exponentiate $D, D^{\prime}, v$, etc. down into $M$ and write $J\left(v_{i}\right)$ for the Jacobian of $T f$ restricted to the tangent plane to $\exp \left(D_{i}\right)$ at $\exp \left(v_{i}\right)$, respecting the smooth Riemann structure; similarly for $J\left(v_{i}^{\prime}\right)$ etc.

3.15. Corollary. On a hyperbolic block, the infinite product $\Pi J\left(q_{i}\right) / J\left(q_{i}^{\prime}\right)$ converges uniformly and the finite products $\prod_{1 \leq i \leq n} J\left(v_{i}\right) / J\left(v_{i}^{\prime}\right)$ stay uniformly bounded as $n \rightarrow \infty$.

Proof. We estimate $\left|J\left(q_{n}\right)-J\left(q_{n}^{\prime}\right)\right|$ as $\leq C\left\{\operatorname{dist}\left(T_{n}, T_{n}^{\prime}\right)\right\}^{\beta}$ where $T_{n}, T_{n}^{\prime}$ are the tangent spaces to $\exp \left(D_{n}\right), \exp \left(D_{n}^{\prime}\right)$ at $\exp \left(v_{n}\right), \exp \left(v_{n}^{\prime}\right)$ and "dist" refers to the distance in the Grassmannian. For $f$ is of class $C^{1+\beta}$. Exponential coordinates distort distance and norm by a bounded amount; we can estimate this distance between tangent planes in the $\exp _{p}$ chart and be sure of our answer up to a factor near 1 . Thus,

$$
\left\{\operatorname{dist}\left(T_{n}, T_{n}^{\prime}\right)\right\} \leq C^{\prime} B_{\varepsilon}\left(f^{n}(p)\right)\left\{\left|q_{n}-q_{n}^{\prime}\right|^{*}+\left\|Q_{n}-Q_{n}^{\prime}\right\|^{*}\right\} \leq C^{\prime \prime}(1+\varepsilon)^{n}\left(\mu^{-\beta^{\prime \prime}}\right)^{n}
$$

and since $\varepsilon \ll \mu-1$, this means that $\left|J\left(q_{n}\right)-J\left(q_{n}^{\prime}\right)\right|$ converges exponentially. Therefore, as above, the corresponding product converges and it does so uniformly over the hyperbolic block. (Note that the constant $C^{\prime \prime}$ includes the value of the $\varepsilon$-slowly varying comparison function $B_{\varepsilon}$ at $p$ and $B_{\varepsilon}$ is constant on the hyperbolic block.) The corresponding analysis for the finite products is similar. Q.E.D.

So far, we have concentrated on the case of hyperbolic orbits, but that was mainly for simplicity. Let $H$ be a Borel set of regular orbits in $M$ and assume the Lyapunov growth rates $\lambda$ for $\mathscr{O} \subset H$ are always different from some 
$\lambda_{0}$. Dividing $H$ into pieces, we may assume that the Lyapunov exponents are bounded away from $\lambda_{0}$ and the pseudo unstable and pseudo stable bundles,

$$
E^{p u} \stackrel{\text { def }}{=} \bigoplus_{\lambda>\lambda_{0}} E^{\lambda}, \quad E^{p s} \stackrel{\text { def }}{=} \bigoplus_{\lambda<\lambda_{0}} E^{\lambda},
$$

have constant dimension. Then we renorm $T_{H} M$ as we did in 3.3 , using $\lambda_{0}$ instead of 1 . We can integrate $E^{p u}$ as follows. First, pull everything back to $H \times X \times Y$ where $H \times X, H \times Y$ are trivialized versions of $E^{p u}, E^{p s}$.

Suppose $\lambda_{0}>1$. Then the graph transform method works quite naturally when we replace the usual norm of a section by the norm

$$
N(\sigma) \stackrel{\text { def }}{=} \sup _{x \neq 0}|s(x)| /|x|,
$$

$s$ being the fiber component of a section $\sigma$ in $\operatorname{Sec}^{\prime}$ that vanishes at $x=0$. See [HPS, p. 54]. The resulting unique $f$-invariant section $\sigma_{f}$, interpreted in $M$, gives the $f$-overflowing pseudo unstable manifold family $\mathscr{W}^{p u}$ tangent to $E^{p u}$. It is unique.

Suppose $\lambda_{0} \leq 1$. Then no unique family $\mathscr{W}^{p u}$ exists, but we can construct one locally $f$-invariant family tangent to $E^{p u}$ as follows. Take the pull-back of $f$ to $H \times X \times Y=E$ which is defined only on $D$ and globalize it to some $\hat{f}: E \circlearrowleft$. Using a slowly varying bump function, it is possible to make $\hat{f} \equiv D f$ near infinity, while the $C^{1}$ difference between $\hat{f}$ and $D f$ remains small. Then follow the $\hat{f}$-graph transform process globally since $\hat{f}$ is global. Since $T^{p s} \hat{f}$ contracts, we continue to deal with bounded sections, vanishing at 0 , under the sup norm. The graph transform produces a unique $\hat{f}$-invariant section $\sigma_{\hat{f}}$. It is $C^{1}$ and its image is tangent to $H \times X$ at 0 . Interpreted in $M, \sigma_{\hat{f}}$ gives a locally $f$-invariant family of $C^{1}$ discs $\mathscr{W}^{p u}$ tangent to $E^{p u}$. The family $\mathscr{W}^{p u}$ depends on which globalization is chosen and it is not unique.

If $f$ is a diffeomorphism then the corresponding analysis of the pseudo stable manifolds proceeds by looking at $f^{-1}$. If $f$ is an endomorphism then the methods of [HPS, $\S 5]$ apply. See also $\S 5$, below.

Next, we state a theorem incorporating these ideas. We assume, as usual, that $f: M \circlearrowleft$ is a $C^{1+\beta}$ diffeomorphism, $0<\beta \leq$ Lip, and $H$ is a Borel set of regular orbits. We assume that the growth rates along orbits in $H$ lie interior to a fixed set of compact intervals $I_{1}, \ldots, I_{l}$ and $I_{l}<I_{l-1}<\cdots<I_{1}$. The notation $I<I^{\prime}$ means that $t<t^{\prime}$ for all $t \in I, t^{\prime} \in I^{\prime}$. For example, if $H$ consists of just one orbit, we could take $I_{i}$ to be a small interval containing the $i$ th growth rate $\lambda_{i}$. Correspondingly, we have a generalized Lyapunov splitting,

$$
T_{H} M=E_{1} \oplus \cdots \oplus E_{l}, \quad E_{i} \stackrel{\text { def }}{=} \bigoplus_{\lambda \in I_{i}} E^{\lambda}, \quad 1 \leq i \leq l .
$$

3.16. Theorem. Tangent to each $E_{i}$ in a generalized Lyapunov splitting is a locally $f$-invariant family of $C^{1+\beta^{\prime}}$ discs $\mathscr{W}_{i}=\left\{W_{i}(z)\right\}_{z \in H}$. 
The number $\beta^{\prime}$ is positive and depends on the spacing of the growth rates. This and the question of higher differentiability of the discs is discussed in $\S 6$. See Theorem 6.6. If $I_{1}>1$ then $\mathscr{W}_{1}$ is unique; if $I_{l}<1$ then $\mathscr{W}_{l}$ is unique.

Sketch of proof. Fix $i$ and group the Lyapunov spaces $E_{j}$ as

$$
E_{i}^{p u}=E_{1} \oplus \cdots \oplus E_{i}, \quad G_{i}=E_{i+1} \oplus \cdots \oplus E_{l} .
$$

According to the preceding discussion there exists a locally $f$-invariant family $\mathscr{W}_{i}^{p u}$ of $C^{1+\beta^{\prime}}$ discs tangent to $E_{i}^{p u}$. Making the same construction for $f^{-1}$ respecting the splitting

$$
E_{i}^{p s}=E_{l} \oplus \cdots \oplus E_{i}, \quad G_{i}^{\prime}=E_{i-1} \oplus \cdots \oplus E_{1}
$$

gives a locally $f^{-1}$-invariant family $W_{i}^{p s}$ tangent to $E_{i}^{p s}$. Local $f^{-1}$-invariance and $f$-invariance are equivalent. By construction the discs $W_{i}^{p u}$ and $W_{i}^{p s}$ form flags $W_{1}^{p u} \subset W_{2}^{p u} \subset \cdots \subset W_{l}^{p u}$, and $W_{1}^{p s} \supset W_{2}^{p s} \supset \cdots \supset W_{l}^{p s}$. Define $W_{i}$ to be the transverse intersection $W_{i}^{p u} \cap W_{i}^{p s}$. Then $W_{i}$ is $C^{1+\beta^{\prime}}$, tangent to $E_{i}$, and $\mathscr{W}_{i}=\left\{W_{i}(z)\right\}$ is locally $f$-invariant. Q.E.D.

Let us apply 3.16 to the case of center manifolds. We assume there are just three intervals $I_{s}<I_{c}<I_{u}$ and $1 \in I_{c}$. Correspondingly, $T_{H} M$ splits as $E^{u} \oplus E^{c} \oplus E^{s}$.

3.17. Corollary: Pesin Center Manifold Theorem. Tangent to $E^{u}, E^{c}, E^{s}$ there are locally $f$-invariant families of $C^{1+\beta^{\prime}}$ discs $\mathscr{W}^{u}, \mathscr{W}^{c}, \mathscr{W}^{s}$. The families $\mathscr{W}^{u}, \mathscr{W}^{s}$ are unique.

Proof. Uniqueness follows because it corresponds to the pseudo unstable case with $\lambda_{0}>1$. Q.E.D.

Finally we remark how this invariant manifold theory extends to flows. Let $f_{t}$ be a $C^{1+\beta}$ flow on $M$. The Oseledec analysis applies to the time-one map $f_{1}$ of the flow, producing a Lyapunov splitting along most orbits. It is invariant under the flow due to its uniqueness and the Chain Rule. Call

$$
E^{u u}=\bigoplus_{\lambda>1} E^{\lambda}, \quad E^{c}=\bigoplus_{\lambda=1} E^{\lambda}, \quad E^{s s}=\bigoplus_{\lambda<1} E^{\lambda} .
$$

The orbit $\mathscr{O}(p)=\left\{f_{t}(p): t \in \mathbf{R}\right\}$ is weak hyperbolic if $E^{c}$ is spanned by the vector field generating the flow. By $3.16,3.17$ there exist unique locally $f$ invariant families of discs $\mathscr{W}^{u u}, \mathscr{W}^{s s}$ tangent to $E^{u u}, E^{s s}$. They are called the strong unstable and strong stable manifolds. By uniqueness and commutativity of the time- $t$-maps of the flow, one checks that for $t>0, f_{t}\left(W^{u u}(p)\right) \supset$ $W^{u u}\left(f_{t} p\right)$ and $f_{t}\left(W^{s s}(p)\right) \subset W^{s s}\left(f_{t} p\right)$. Hence,

3.18. Corollary. Along weak hyperbolic orbits of a flow, strong unstable and strong stable manifolds exist, are unique, and are of class $C^{r+\beta^{\prime}}$. 


\section{Absolute continuity}

In this section, we prove Theorem 2 -absolute continuity of the $\mathscr{W}^{u}$ and $\mathscr{W}^{s}$ laminations. Our proof is a modification of what we did in our treatment of the absolute continuity of the horocycle foliations for Anosov actions in [PS1]. The biggest obstacle here is that the Three Discs Lemma of Anosov [A] is not available. Instead, we use an overcovering argument; see 4.1. In fact the proof we give below provides an alternate proof of absolute continuity in the uniformly hyperbolic Anosov case.

As usual, we assume the diffeomorphism $f: M \supset$ is of class $C^{1+\beta}, 0<$ $\beta \leq \mathrm{Lip}$, and $\mathscr{O}$ is a weakly hyperbolic orbit. Through $q, q^{\prime} \in W^{u} p, p \in \mathscr{O}$, draw $s$-dimensional, smooth discs $D$ and $D^{\prime}$ transverse to $W^{u} p$. We want to investigate the $\mathscr{W}^{u}$ holonomy map from $D$ to $D^{\prime}$. See $\S 1$ and Figure 1.

Let us first make a motivating digression about what we could do if differentiability and uniformity problems were trivial. Imagine that $\mathscr{W}^{u}$ is a $C^{\infty}$ foliation. Then it induces a smooth holonomy map $h: D \rightarrow D^{\prime}$ and we could try to calculate its Jacobian at $q$; this is exactly what we want to do even when $\mathscr{W}^{u}$ is neither smooth nor a foliation. Imagine also that $\mathscr{V}$ is another $C^{\infty} u$ dimensional foliation whose leaves are fairly near those of $\mathscr{W}^{u}$ in the $C^{1}$ sense. Imagine that we have been told exactly what are the Jacobians of the holonomy maps along the $\mathscr{V}$ foliation (at all pairs of points $q, q^{\prime}$ ) and imagine that in terms of them we hope to calculate the Jacobian of $h$.

Under $f$-iteration we can expect $f^{n} \mathscr{V} \rightrightarrows \mathscr{W}^{u}$ as $n \rightarrow \infty$. (This is the utility of the graph transform method.) Thus, the Jacobians along $f^{n} \mathscr{V}, h_{n}: D \rightarrow D^{\prime}$, can be expected to converge to $J(h)$, the Jacobian of $h$. Another way to express $J\left(h_{n}\right)$ is by commutativity of the diagram

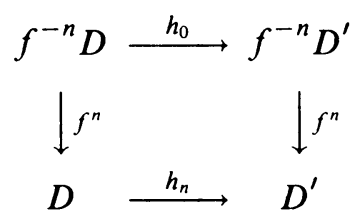

where $h_{0}$ is the uniterated $\mathscr{V}$-holonomy. Thus,

$$
J\left(h_{n}\right)=J_{q_{n}^{\prime}}\left(f^{n}\right) J_{q_{n}}\left(h_{0}\right) J_{q}\left(f^{-n}\right)
$$

where $q_{n}=f^{-n}(q), q_{n}^{\prime}=h_{0} f^{-n}(q)$, and $J\left(f^{-n}\right), J\left(f^{n}\right)$ are the Jacobians of the restriction of $f^{-n}, f^{n}$ to the appropriate inverse image of $D$ or $D^{\prime}$. The beauty of Jacobians, of course, is that they regulate infinitesimal area-change and, being real numbers, they commute. Also, the chain rule for derivatives becomes a product formula for Jacobians.

We have said that it is reasonable to expect $J\left(h_{n}\right) \rightarrow J(h)$. Thus, to calculate $J(h)$ we would "only" have to know $J\left(f^{-n}\right)$ at various points and $J\left(h_{0}\right)$ at $q_{n}$. This last factor should tend to 1 because, since $q_{n}$ and $q_{n}^{\prime}$ are asymptotic as $n \rightarrow \infty$, the $\mathscr{V}$-holonomy expressed by $h_{0}$ takes place across a shorter and 
shorter distance. Taking the limit and commuting the Jacobians, one would predict that

$$
J_{q}(h)=\prod_{i=1}^{\infty} \frac{J_{f^{-i} q^{\prime}}(f)}{J_{f^{-i} q}(f)} .
$$

In fact, this formula is true in the weak hyperbolic case, but we are going to prove something easier - the product above converges and a multiple of it bounds the Radon Nikodym derivative of the holonomy.

First we state and prove a covering lemma that may well be a standard fact, since its proof is so straightforward. Let $K$ be a subset of an $s$-dimensional metric space $W$. We want to cover $K$ efficiently by $\delta$-neighborhoods-we want few of the neighborhoods to overlap. We assume that the neighborhood of $K$ is quasi-Euclidean at scale $\delta$ in the sense that for each $p \in K$ there exists a homeomorphism $\varphi$ from the $4 \delta$-disc in $\mathbf{R}^{S}$ onto a neighborhood of $p$ in $W$ such that $\varphi(0)=p$ and $\varphi$ has low distortion:

$$
3 / 4 \leq d\left(\varphi(x), \varphi\left(x^{\prime}\right)\right) /\left|x-x^{\prime}\right| \leq 4 / 3 .
$$

4.1. Overcovering Lemma. $K$ can be covered by $\delta$-neighborhoods in $W$ such that fewer than $8^{s}$ of them overlap at any point.

Proof. Let $A$ be a maximal $\delta$-separated set in $K$; i.e., $d\left(a, a^{\prime}\right) \geq \delta$ for all distinct $a, a^{\prime} \in A$ and no point $p \in K$ is farther than $\delta$ from $A$. It is a Zorn's lemma argument to prove that $A$ exists. Then $\left\{W_{\delta}(a)\right\}$ covers $K$ and the $\frac{1}{2} \delta$ subneighborhoods of the $a \in A$ are disjoint. Fix any $a_{0} \in A$ and suppose that $N-1$ of the $W_{\delta}(a)$ intersect $W_{\delta}\left(a_{0}\right)$, say $a=a_{i}, i=1, \ldots, N-1$. Then $W_{3 \delta}\left(a_{0}\right)$ contains the $N$ disjoint sets $W_{\delta / 2}\left(a_{i}\right), i=0, \ldots, N-1$. The image of the quasi-Euclidean homeomorphism $\varphi_{0}$ at $a_{0}$ contains $W_{3 \delta}$ because $\varphi_{0}$ distorts distance by at worst the factor $\frac{3}{4}$. Thus, we get $N$ disjoint sets $\varphi_{0}^{-1}\left(W_{\delta / 2}\left(a_{i}\right)\right)$, each of which contains a Euclidean disc $\Delta_{i}$ of radius $3 \delta / 8$. See Figure 8. Therefore, $N$ area $\left(D_{3 \delta / 8}\right)<\operatorname{area}\left(D_{3 \delta}\right)$, and hence $N<8^{s}$. Q.E.D.

Proof of Theorem 2: Absolute continuity. We prove absolute continuity of the $\mathscr{W}^{u}$-holonomy maps. It suffices to do so locally for the $\mathscr{W}_{P}^{u}$-holonomy maps, $P$ being a single, small hyperbolic block. (By $\mathscr{W}_{P}^{u}$ we refer to the lamination of unstable manifolds based at points of $P$.) For by 3.6 and 3.13, $\mathscr{W}^{u}$ is the union of $f^{k} \mathscr{W}_{P}^{u}$ over countably many iterates of countably many hyperbolic blocks, and all questions of measure zero are insensitive to countable union. Likewise, global holonomy maps are built up from local ones by composition. In particular, we need only deal with hyperbolic blocks contained in $\mathscr{H}(\tau)$, the set of weak hyperbolic orbits whose growth rates lie outside the interval $\left[\tau^{-1}, \tau\right]$.

Choose $\mu$ and $\varepsilon, 1<(1+\varepsilon)^{3}<\mu<\tau$, and let ||$^{*}$ be the adapted Finsler supplied by 3.3. By 3.13, applied to $f^{-1}$, we may assume that on the saturate $H$ of our hyperbolic block $P$ there are defined $\varepsilon$-slowly varying Borel radius functions, $r$ and $r^{\prime}$, and $\bar{W}_{z}^{s}(r)$ contains a fairly flat $s$-disc $G_{z}$ of radius 


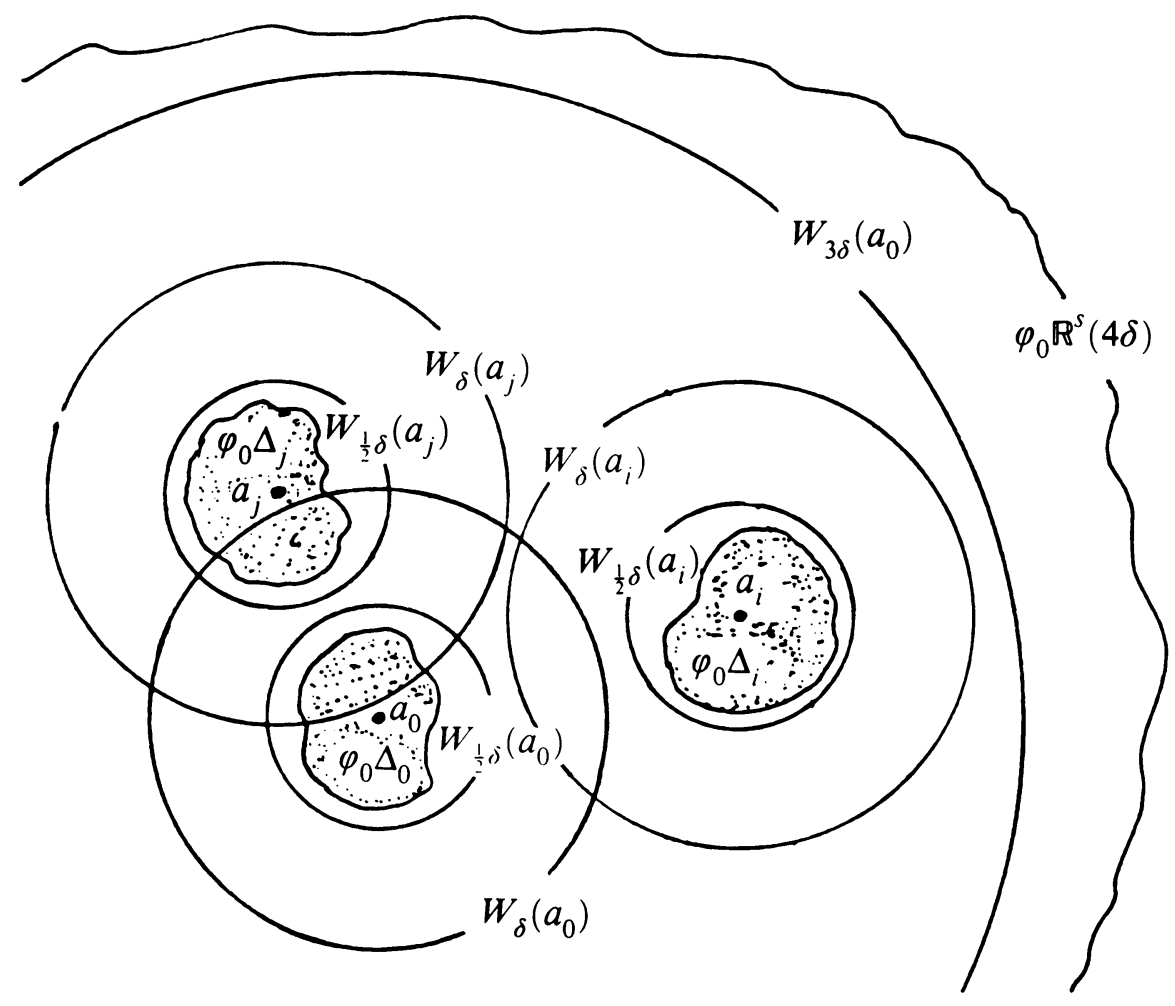

Figure 8. Controlled overlap

$r^{\prime}(z), z \in H$. The $s$-disc $G_{z}$ is the graph of some map $g_{z}: E_{z}^{s}\left(r^{\prime}\right) \rightarrow E_{z}^{s \perp}\left(r^{\prime}\right)$ and $\left|g_{z}\right|_{C^{\prime}} \rightrightarrows 0$ as $|k| \rightarrow \infty, z \in f^{k} P$.

Now consider $p_{0} \in P$ and draw smooth discs $D, D^{\prime}$ of dimension $s=$ $m-u$ which cross $W_{p_{0}}^{u}(r)$ transversely. Because $P$ is small and $\mathscr{W}^{u}$ is $C^{1}$ continuous on $P$, we may also assume that each $W_{p}^{u}(r), p \in P$, crosses $D$ and $D^{\prime}$ transversely at unique points, say $y$ and $y^{\prime}$. We call the union of these points $y=W_{p}^{u}(r) \cap D$, the shadow of $\mathscr{W}_{P}^{u}$ on $D$, and denote it by $S$. The $\mathscr{W}_{P}^{u}$-holonomy map is a homeomorphism $h: S \rightarrow S^{\prime}, y \mapsto y^{\prime}$ where $S^{\prime}$ is the shadow of $\mathscr{W}_{P}^{u}$ on $D^{\prime}$. (Although $S, S^{\prime}$ need not be compact, $h$ extends continuously to a homeomorphism from $\bar{S}$ onto $\bar{S}^{\prime}$.) We may assume that $P$ is small enough and $D, D^{\prime}$ are close enough together that $S, S^{\prime}$ are bounded away from $\partial D, \partial D^{\prime}$. This is what we mean by $h$ being a local holonomy map. See Figure 1 in $\S 1$.

If $Z \subset S \subset D$ and $Z$ has (Riemann) measure zero then we must show that $h(Z)$ has measure zero in $D^{\prime}$. First we show that the neighborhood of $f^{-n} S$ in $f^{-n} D$ is quasi-Euclidean at scale $\delta_{n}$, where $\delta_{n}=\frac{1}{2} r^{\prime}\left(f^{-n} p\right)$. The natural metric on $f^{-n} D$ is $d\left(x, x^{\prime}\right)=$ the infimum of the arc-lengths of $C^{1}$ paths in $f^{-n} D$ that join $x$ to $x^{\prime}$, the smooth Finsler || being used to measure 
arc-length. We must find low distortion homeomorphisms $\varphi$ of $\mathbf{R}^{s}\left(4 \delta_{n}\right)$ onto neighborhoods of points of $f^{-n} S$ in $f^{-n} D$ as in 4.1. See Figure 9.

Let $D, D^{\prime}$ be the lifts of $D, D^{\prime}$ to $T_{p} M, \bar{D}=\exp _{p}^{-1}(D), D^{\prime}=\exp _{p}^{-1}\left(D^{\prime}\right)$. We think of them as trial $s$-discs in $T_{p} M$. When it is simpler, we write $r_{n}^{\prime}$ for $r^{\prime}\left(f^{-n} p\right), W_{n}^{s}$ for $W_{f^{-n} p}^{s}$, etc. Call $y_{n}=f^{-n}(y)$ and $\bar{y}_{n}=\exp _{n}^{-1}\left(y_{n}\right)$, where $y=W_{p}^{u}(r) \cap D$. Thus, $\bar{f}\left(\bar{y}_{n}\right)=\bar{y}_{n-1}, \bar{y}_{n} \in \bar{W}_{n}^{u}(r)$, and $\left|\bar{y}_{n}\right|^{*} \rightarrow 0$ at an exponential rate $(1+\varepsilon) / \mu$. Since ||$^{*}$ is comparable to || be an $\varepsilon$-slowly varying function $B_{\varepsilon},\left|\bar{y}_{n}\right| \rightarrow 0$ at rate $(1+\varepsilon)^{2} / \mu$. Since $(1+\varepsilon)^{3}<\mu$, this rate is faster than the rate at which the radius $r_{n}^{\prime}$ may shrink. Thus, $\bar{y}_{n}$ lies in (and very near the center of) the fairly flat $s$-disc $G_{n} \subset f^{-n} D$ that is supplied by 3.13; in particular, $\pi^{s}\left(\bar{y}_{n}\right) \in E_{n}^{s}\left(r^{\prime}\right)$ where $\pi^{s}$ is the orthogonal projection of $T_{H} M$ onto $E^{s}$. Let $i_{n}: \mathbf{R}^{s} \rightarrow E_{n}^{s}$ be an isometry that sends 0 to $\pi^{s}\left(\bar{y}_{n}\right)$ and define $\varphi$ as the composition

$$
\varphi(x)=\exp _{n} \circ \operatorname{graph}\left(g_{n}\right) \circ i_{n}(x), \quad x \in \mathbf{R}^{s}\left(4 \delta_{n}\right),
$$

where $G_{n}$ is the graph of $g_{n}$. Since $\left|\bar{y}_{n}\right| \rightarrow 0$ at a faster rate than $\delta_{n} \rightarrow$ $0, i_{n}\left(\mathbf{R}^{s}\left(4 \delta_{n}\right)\right) \subset E_{n}^{s}\left(r^{\prime}\right)$. Since $\left|g_{n}\right|_{C^{1}} \rightrightarrows 0$ as $n \rightarrow \infty$, by 3.13 , it follows that $\varphi$ distorts arc-length very little. That is, $\varphi$ has low distortion and the neighborhood of $f^{-n} S$ in $f^{-n} D$ is quasi-Euclidean at scale $\delta_{n}$, for $n$ large. The same is true of $f^{-n} S^{\prime}$ in $f^{-n} D^{\prime}$.

Now suppose that $Z \subset S \subset D$. and $Z$ has (Riemann) measure zero. We must show that $h(Z)$ has measure zero in $D^{\prime}$. We write $|A|$ to indicate the Riemann measure of a set $A$, if no confusion arises. It is enough to prove that there is a constant $J$ such that for any small disc $\Delta \subset D,|h(\Delta)| \leq J|\Delta|$. For

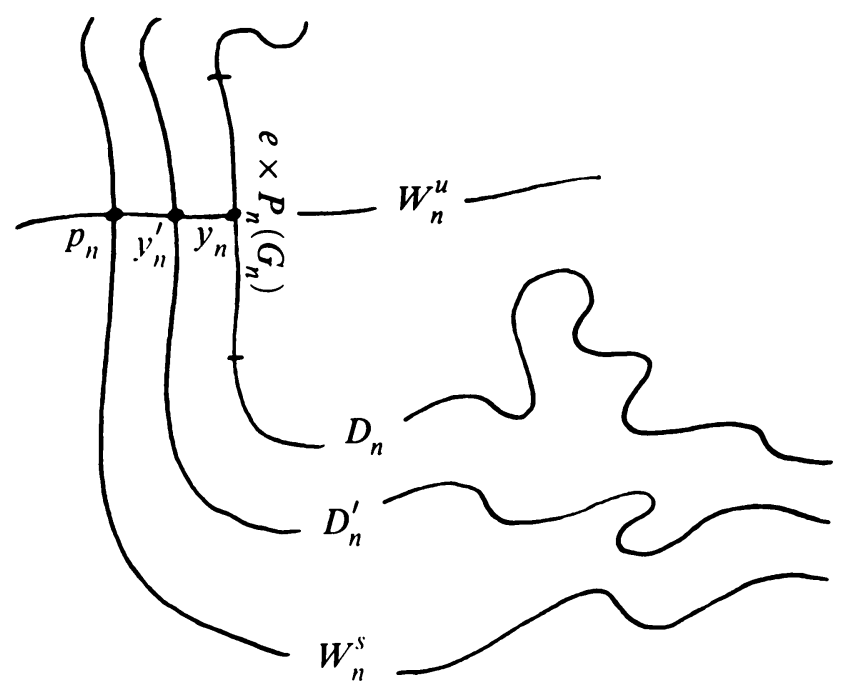

FiguRE 9. Fairly flat parts of ugly discs in $M$ 
the zero set $Z \subset S$ can be covered by countably many of such $\Delta$ 's whose total measure is $<\varepsilon / J$. Then $h(Z)$ is covered by countably many sets $h(\Delta)$ whose total measure is $<\varepsilon$; hence $|h(Z)|=0$. Instead of $h(\Delta)$ it is more accurate to write $h(\Delta \cap S)$. We assume $\Delta$ is small enough that the disc of twice its radius at the same center still lies in $D$.

Since the neighborhood of $f^{-n} S$ in $f^{-n} D$ is quasi-Euclidean at scale $\delta_{n}$, the same is true for any subset of $f^{-n} S$; e.g., $f^{-n}(\Delta \cap S)$. By 4.1, $f^{-n}(\Delta \cap S)$ can be covered by discs $R_{i}$ of radius $\delta_{n}$ in such a way that at most $8^{s}$ of them overlap. We are going to over cover $h_{n}\left(R_{i} \cap S_{n}\right)=R_{i}^{\prime}$, where $S_{n}=f^{-n} S$ and $h_{n}: S_{n} \rightarrow S_{n}^{\prime}$ is the $\mathscr{W}_{n}^{u}$-holonomy map, $h_{n}=f^{-n} \circ h \circ f^{n}$. Note that $d\left(w, h_{n} w\right) \rightarrow 0$ at a uniform rate $\left((1+\varepsilon)^{2} / \mu\right)^{n}$ where $w \in f^{-n} S$, since $P$ is a hyperbolic block. Here, $d\left(w, h_{n} w\right)$ refers to the smooth Riemann metric on $M$.

Take any $R_{i}$, double its radius, call the answer $\widehat{R}_{i}$, and "push it across" from $f^{-n} D$ to $f^{-n} D^{\prime}$ as follows. Let $y_{n}=f^{-n}(y)$ be the center of $R_{i}$, and call $y_{n}^{\prime}=f^{-n}\left(y^{\prime}\right)$ where $h(y)=y^{\prime}$. Since $f^{-n} D$ and $f^{-n} D^{\prime}$ contain fairly flat $s$-discs $G_{n}$ and $G_{n}^{\prime}$ of radius $r_{n}^{\prime}$ at $y_{n}$ and $y_{n}^{\prime}$, there exists a $u$-plane in $T M$ whose parallel translates near $\widehat{R}_{i}$ are approximately perpendicular to $\widehat{R}_{i}$. We push along it, from $f^{-n} D$ to $f^{-n} D^{\prime}$. The distance we push across is not significantly more than the distance from $y_{n}$ to $y_{n}^{\prime}$, and this is substantially less than the radius of the disc being pushed. During such a short, nearly perpendicular push, distance is changed very little. (Shape, on the other hand, is changed a great deal.) Thus, when pushed across to $f^{-n} D^{\prime}, \widehat{R}_{i}$ becomes a neighborhood of $y_{n}^{\prime}, \widehat{R}_{i}^{\prime}$, and $\widehat{R}_{i}^{\prime}$ contains a disc at $y_{n}^{\prime}$ of radius $3 \delta_{n} / 2$. But $h_{n}\left(R_{i} \cap S_{n}\right)=R_{i}^{\prime}$ is contained in a disc at $y_{n}^{\prime}$ whose radius is nearly $\delta_{n}$. (The radius is no more than $\delta_{n}+d\left(\mathrm{id}_{n}, h_{n}\right)$ where $\mathrm{id}_{n}: f^{-n} S \rightarrow M$ is the inclusion map and $d\left(\mathrm{id}_{n}, h_{n}\right) \rightarrow 0$ at a faster rate than $\delta_{n} \rightarrow 0$.) Hence, $h_{n}\left(R_{i}\right) \subset \widehat{R}_{i}^{\prime}$. See Figure 10.

Since $h_{n}\left(R_{i}\right) \subset \widehat{R}_{i}^{\prime},\left\{\widehat{R}_{i}^{\prime}\right\}$ is a covering of $f^{-n}(h(\Delta \cap S))$. The volume of $\widehat{R}_{i}^{\prime}$ is comparable to that of $R_{i}$ because pushing across is a smooth operation applied to a well-controlled object and doubling the radius multiplies the volume by approximately $2^{s}$. That is, $\left|\hat{R}_{i}^{\prime}\right| /\left|R_{i}\right|$, is uniformly bounded, say by $R$.

Now for $n$ large we estimate $\sum_{i}\left|f^{n}\left(\widehat{R}_{i}^{\prime}\right)\right|$. By 3.15 , the Jacobian of $f^{n}$ at any point of $\widehat{R}_{i}$ is comparable to the Jacobian of $f^{n}$ at a point of $\hat{R}_{i}^{\prime}$. That is, $J\left(f^{n}, z^{\prime}\right) / J\left(f^{n}, z\right)$ is bounded, say by $J_{0}$, for $z \in \widehat{R}_{i}, z^{\prime} \in \widehat{R}_{i}^{\prime}$. Thus,

$$
\left|f^{n}\left(\widehat{R}_{i}^{\prime}\right)\right| /\left|f^{n}\left(R_{i}\right)\right| \leq R J_{0} .
$$

At most $8^{s}$ of the $f^{n}\left(R_{i}\right)$ overlap and all of them are contained in the double of $\Delta$, say $\widehat{\Delta}$. Their total volume is at most $8^{s}|\widehat{\Delta}|$. The volume of $\widehat{\Delta}$ is approximately $2^{s}|\Delta|$. Hence,

$$
|h(\Delta)| \leq \sum_{i}\left|f^{n} R_{i}^{\prime}\right| \leq \sum_{i}\left|f^{n} \widehat{R}_{i}^{\prime}\right| \leq R J_{0} \sum_{i}\left|f^{n} R_{i}\right| \leq R J_{0} 8^{s} 2^{s}|\Delta| .
$$




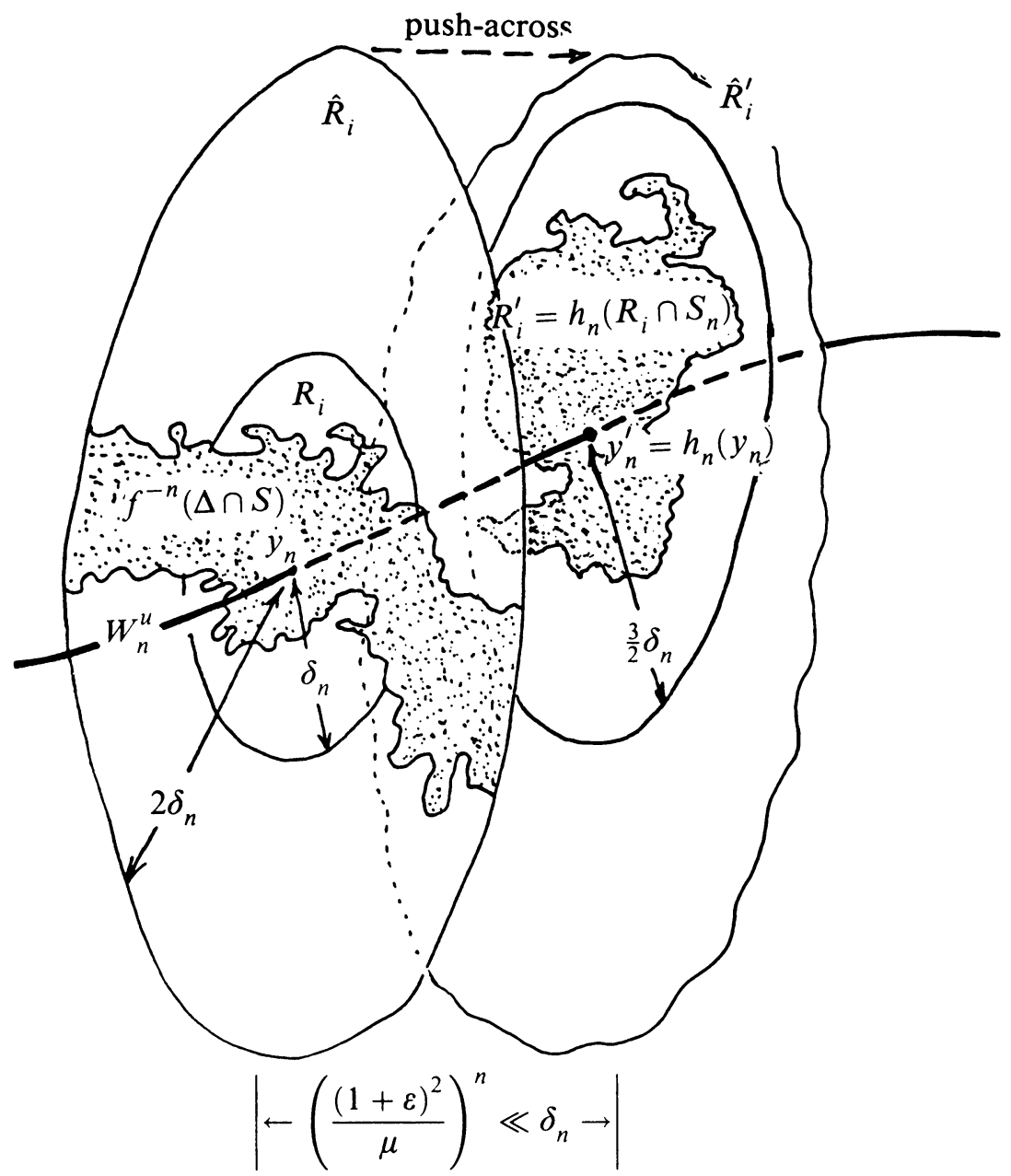

FiguRE 10. When pushed across, $\widehat{R}_{i}$ becomes $\widehat{R}_{i}^{\prime}$ and $\widehat{R}_{i}^{\prime}$ still covers $R_{i}^{\prime}=h_{n}\left(R_{i} \cap S_{n}\right), S_{n}=f^{-n} S$ being the $n$th shadow

Thus, $J=R J_{0}(16)^{s}$ is a constant that compares the volume of $h(\Delta)$ and $\Delta$. From the existence of $J$ we know that $h$ is absolutely continuous. Q.E.D.

\section{ENDOMORPHISMS}

The stable and unstable manifold theorems are theorems in the neighborhood of an orbit. If $f: M \rightarrow M$ is not a diffeomorphism we have comparable theorems in the neighborhood of a full orbit, i.e. a bi-infinite sequence $\left\{x_{i}\right\} \in Z$ such that $x_{j}=f^{j-i}\left(x_{i}\right)$ for any $j>i .^{2}$ It is not even necessary that $f$ be a local diffeomorphism at the $x_{i}$, that is $T_{x_{i}} f$ need not be an isomorphism. We

${ }^{2}$ Actually a stable manifold theorem can be stated for positive half orbits and an unstable manifold theorem can be stated for negative half orbits. 
reverse the order of definitions so that we may proceed directly to the stable manifold theorem.

Definition. We say that the full orbit $\left\{x_{i}\right\}_{i \in Z}$ of the endomorphism $f: M \rightarrow M$ is weak hyperbolic if there is a $T f$-invariant splitting $T_{x_{i}} M=E_{x_{i}}^{s} \oplus E_{x_{i}}^{u}$. A constant $0<\lambda<1$ and for any $\varepsilon>0$, functions $A_{\varepsilon}\left(x_{i}\right)$ such that

(a) (i) $\left|T_{x_{i}} f^{n}(v)\right| \leq A_{\varepsilon}\left(x_{i}\right) \lambda^{n}|v|$ for all $v \in E_{x_{i}}^{s}$ and all $n \geq 0$,

(a) (ii) $\left|T_{x_{i}} f^{-n}(v)\right| \leq A_{\varepsilon}\left(x_{i}\right) \lambda^{n}|v|$ for all $v \in E_{x_{i}}^{u}$ and all $n \geq 0$, and $T_{x_{i}} f^{n} \mid E_{x_{i}}^{u}$ is invertible so (ii) makes sense.

(b) $\varangle\left(E_{x_{i}}^{s}, E_{x_{i}}^{u}\right)^{-1} \leq A_{\varepsilon}\left(x_{i}\right)$.

(c) $A_{\varepsilon}\left(x_{j}\right) \leq(1+\varepsilon)^{|j-i|} A_{\varepsilon}\left(x_{i}\right)$.

The unstable and stable manifold theorems remain essentially unchanged for asymptotically hyperbolic orbits of endomorphisms. Here we use $C^{r+(\beta-)}$ for $\beta>0$ to mean $C^{r+\beta^{\prime}}$ for all $0 \leq \beta^{\prime}<\beta$.

5.1. Theorem. Let $f: M \rightarrow M$ be $C^{1+\beta}$ and $\left\{x_{i}\right\}_{i \in Z}$ an asymptotically hyperbolic orbit. Then there are maps

$$
\begin{gathered}
w^{u}\left(x_{i}\right) \in C^{1+(\beta-)}\left(E_{x_{i}}^{u}\left(r_{u}\left(x_{i}\right)\right), E_{x_{i}}^{s}\left(r_{u}\left(x_{i}\right)\right)\right), \\
w^{s}\left(x_{i}\right) \in C^{1+(\beta-)}\left(E_{x_{i}}^{s}\left(r_{s}\left(x_{i}\right)\right), E_{x_{i}}^{u}\left(r_{s}\left(x_{i}\right)\right)\right)
\end{gathered}
$$

such that $r_{u}, r_{s}$ are slowly varying functions of $x_{i}$.

(ii) $W_{r}^{u}\left(x_{i}\right)=\exp _{x_{i}}$ (graph $\left.w^{u}\left(x_{i}\right)\right)$ is a family of $f$-overflowing invariant discs through $x_{i}, f\left(W_{r}^{u}\left(x_{i}\right)\right) \supset W_{r}^{u}\left(f\left(x_{i}\right)\right)$ tangent at $x_{i}$ to $E_{x_{i}}^{u}$. Up to restriction $W_{r}^{u}\left(x_{i}\right)$ is unique. The points of $W_{r}^{u}\left(x_{i}\right)$ are characterized by $z \in W_{r}^{u}\left(x_{i}\right)$ iff $d\left(z, x_{i}\right)<r\left(x_{i}\right)$ and for $j<i \exists z_{j}$ with $d\left(z_{j}, x_{j}\right) \rightarrow 0$, $d\left(z_{j}, x_{j}\right)<r\left(x_{j}\right)$ and $f^{i-j}\left(z_{j}\right)=z$. In this case $d\left(z_{j}, x_{j}\right) \lambda^{-n} \rightarrow 0$. Any family of "trial" unstable manifolds $\left\{W^{\prime}\left(x_{i}\right)\right\}$ reasonably close to $\left\{W_{r}^{u}\left(x_{i}\right)\right\}$ locally converges to $\left\{W_{r}^{u}\left(x_{i}\right)\right\}$ under $f^{n}$ iterations as $n \rightarrow$ $\infty$. The convergence occurs in the $C^{1+(\beta-)}$ sense.

(iii) $W_{r}^{s}\left(x_{i}\right)=\exp _{x_{i}}$ (graph $w^{s}\left(x_{i}\right)$ ) is a family of $f$-invariant discs through $x_{i}, f\left(W_{r}^{s}\left(x_{i}\right)\right) \subset W_{r}^{s}\left(f\left(x_{i}\right)\right)$ tangent at $x_{i}$ to $E_{x_{i}}^{s}$. Up to restriction $W_{r}^{s}\left(x_{i}\right)$ is unique. The points of $W_{r}^{s}\left(x_{i}\right)$ are characterized by $z \in W_{r}^{s}\left(x_{i}\right)$ iff $d\left(f^{n}\left(x_{i}\right), f^{n}(z)\right)<r\left(x_{i+n}\right)$ for all $n \geq 0$ and in this case $d\left(f^{n}\left(x_{i}\right), f^{n}(z)\right) \lambda^{-n} \rightarrow 0$. Any family of "trial" stable manifolds $\left\{W^{\prime}\left(x_{i}\right)\right\}$ reasonably close to $\left\{W_{r}^{s}\left(x_{i}\right)\right\}$ locally converges to $\left\{W_{r}^{s}\left(x_{i}\right)\right\}$ under inverse graph transform.

(iv) If $f$ is $C^{r}$ for $r \geq 2$ or $C^{r+\beta}$ for $r \geq 1$ then the stable and unstable manifolds are $C^{r}$ and $C^{r+(\beta-)}$.

Proof. The proof of the theorem is precisely as the Unstable Manifold Theorem. The renorming is similarly accomplished. It is irrelevant that $T f^{-1} \mid E^{s}$ exist or 
be bounded. The distances are measured in the max metric in $T_{x_{j}} M$. The only difference is that graph transform is defined slightly differently for the stable manifolds as in [HPS, p. 56].

This is the graph transform approach alluded to in [RS]. A more general theorem for endomorphisms is true for any split in the exponents as for diffeomorphisms but we leave it as an exercise for the reader. An almost everywhere stable and unstable manifold theorem is true for endomorphisms as well. This is proven by [Ru and M1] even in infinite dimensions for compact operations and was announced in [RS]. It depends on an Oseledec theorem for endomorphisms; we state a theorem with the functions $A_{\varepsilon}$ included. We rely on [M1 and $\mathrm{Ru}$ ] and follow the notation of [FHY]. It is simpler to work on full orbits so we let $\widetilde{M}=\lim _{f} M_{f}$ be the inverse limit, that is the subset of $\Pi M$ consisting of full orbits. A point in $\widetilde{M}$ is a sequence. Let $\tilde{f}: \widetilde{M} \rightarrow \widetilde{M}$ be the induced map. $\tilde{f}$ is a homeomorphism; it is simply the shift map. If we let $\Pi: \widetilde{M} \rightarrow M$ be defined by $\tilde{\Pi}\left(\left\{x_{i}\right\}_{i \in Z}\right)=x_{0}$, $\tilde{\Pi} \tilde{f}=f \tilde{\Pi}$ and $\tilde{\Pi}$ maps the set of invariant Borel probability measures for $\tilde{f}$ onto the set of invariant Borel probability measures for $f$. The tangent bundle of $M, T M$ pulls back to $T \widetilde{M}$ on $\widetilde{M}$ and $T f$ extends to $\tilde{T} f$ :

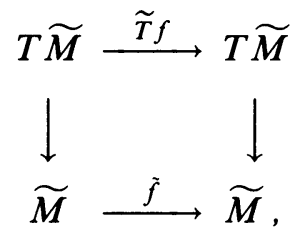

$\tilde{T} f$ is a continuous bundle endomorphism covering the homeomorphism $\tilde{f}$ of the compact base so $\widetilde{T} f$ is a linear map on each fiber and $\exists K>0$ such that $\|\tilde{T} f\|<K$ for any $\tilde{m} \in \widetilde{M}$.

5.2. Theorem. There is a Borel set $\tilde{\Gamma} \subset \widetilde{M}$ which has the following properties:

(i) $\tilde{\Gamma}$ is invariant under $\tilde{f}$ and has measure 1 for every $\tilde{f}$-invariant probability measure on $\widetilde{M}$.

(ii) For every $\tilde{m} \in \tilde{\Gamma}, \tilde{m}=\left(m_{i}\right)_{i \in Z}$ there is an integer $k$ and a $T f$-invariant splitting of the tangent space

$$
T_{m_{i}} M=E_{1}\left(m_{i}\right) \oplus \cdots \oplus E_{k}\left(m_{i}\right) \oplus F_{\infty}\left(m_{i}\right)
$$

and numbers $\lambda_{1}(\tilde{m})>\cdots>\lambda_{i}(\tilde{m})$ such that

(a) The $E_{i}$ 's, $F_{\infty}$ and $\lambda$ 's are Borel measurable functions.

(b) $T f \mid E_{f}\left(m_{i}\right)$ is an isomorphism $1 \leq j \leq k$.

(c) $\lim _{n \rightarrow+\infty} \frac{1}{n} \log |\widetilde{T} f v|=\lambda_{j}\left(\tilde{m}_{i}\right)$ for all $0 \neq v \in E_{j}\left(m_{i}\right), \quad 1 \leq j \leq k$.

(d) $\lim \frac{1}{n} \log \left|\tilde{T} f^{n}\right| F_{\infty}\left(m_{i}\right) \mid=-\infty$.

(e) If $0 \neq v_{i} \in F_{\infty}\left(m_{i}\right)$ and there are $v_{j} \in F_{\infty}\left(m_{j}\right)$ for $j<i$ such that $T f^{i-j}\left(v_{j}\right)=v_{i}$ then $\lim _{j \rightarrow \infty} \frac{1}{j} \log \left|v_{j}\right|=-\infty$. 
(f) $F_{\infty}\left(m_{i}\right)=K\left(m_{i}\right) \oplus G_{\infty}\left(m_{i}\right)$ where $T_{m_{i}} f^{n_{i}} \mid K\left(m_{i}\right)$ is identically 0 for some $n_{i}$ and $T f \mid G_{\infty}\left(m_{i}\right) \rightarrow G_{\infty}\left(m_{i+1}\right)$ is an isomorphism.

(g) Given $\varepsilon>0$ there is a Borel measurable function $A_{\varangle}: \tilde{\Gamma} \rightarrow(1, \infty)$ such that for all $\tilde{m} \in \tilde{\Gamma}$

(i) $\varangle\left(E_{j}\left(m_{i}\right), E_{l}\left(m_{i}\right)\right)^{-1} \leq A_{\varangle}\left(m_{i}\right), \varangle\left(\bigoplus_{j=1}^{k} E_{j}\left(m_{i}\right), F_{\infty}\left(m_{i}\right)\right)^{-1} \leq A_{\varangle}\left(m_{i}\right)$, and $A_{\varangle}\left(m_{j}\right) \leq(1+\varepsilon)^{|j-i|} A_{\varangle}\left(m_{i}\right)$.

(h) Let $\lambda \in \mathbf{R}$. Denote by $B_{\lambda, \varepsilon}$ the subset of $\tilde{\Gamma}$ which consists of points $\tilde{m}$ such that $\lambda_{i}(\tilde{m}) \notin[\lambda-\varepsilon, \lambda+\varepsilon], i=1, \ldots, k(\tilde{m})$. This set is f-invariant. For $\tilde{m} \in B_{\lambda, \mu}$ let $E_{\tilde{m}}^{s}=\bigoplus_{\lambda_{i}<\lambda} E_{i}(\tilde{m})$ and $E_{\tilde{m}}^{u}=$ $\bigoplus_{\lambda<\lambda_{i}} E_{i}(\tilde{m})$. Then there exists a real valued Borel measurable function $A_{\varepsilon}$ defined on $B_{\lambda, \varepsilon}$ such that for every $\tilde{m}$ in $B_{\lambda, \varepsilon}$

(i) $\forall v \in E_{\tilde{m}}^{s}, \forall n \geq 0,\left|T_{m_{0}} f^{n}(v)\right| \leq A_{\varepsilon}(\tilde{m})|v| e^{(\lambda-\varepsilon) n}$,

(ii) $\forall v \in E_{\tilde{m}}^{u}, \forall n \geq 0,\left|T_{m_{0}} f^{-n}(v)\right| \leq A_{\varepsilon}(\tilde{m})|v| e^{-(\lambda-\varepsilon) n}$,

(iii) $\forall n \in \mathbb{Z}, A_{\varepsilon}\left(f^{n}(\tilde{m})\right) \leq A_{\varepsilon}(\tilde{m}) e^{\varepsilon|n|}$.

We sketch a proof of this theorem, relying on all the linear theory already in [M1 or RS]. Let $K(m)=\left\{v \in T_{m} M \mid T f^{n}(v)=0\right.$ for some $\left.n>0\right\}$. This is the "eventual kernel" at $m$. Thus $\operatorname{dim} K(f(m)) \leq \operatorname{dim} K(m)$ since $T f_{m}: K(m) \rightarrow K(f(m))$ and in fact $K(m)=\left(T_{f(m)} f\right)^{-1} K(f(m))$. If we consider the set of points such that $\operatorname{dim} K(f(m))<\operatorname{dim} K(m)$ then we see by Poincare recurrence that the measure of this set is 0 for any invariant measure $M$. Similarly we let $V(\tilde{m})=\bigcap T_{m_{-n}} f^{n}\left(T_{m_{-n}} M\right)$ for $\tilde{m} \in \widetilde{M}$ and $n \geq 0$. This is the ultimate image. $V(\tilde{m}) \subset T_{m_{0}} M$ and $\tilde{T} f V(\tilde{m}) \subset V(\tilde{f} m)$, since $T_{m_{0}} f: T_{m_{0}} M \rightarrow T_{f m_{0}} M$. Now $V(\tilde{m})$ can drop dimension along orbits only on sets of measure 0 again by Poincaré recurrence and we may suppose that $\widetilde{T} M_{\tilde{m}}=K(\tilde{m}) \oplus V(\tilde{m}) . T f_{\tilde{m}}: V(\tilde{m}) \rightarrow V(\tilde{f}(\tilde{m}))$ is an isomorphism of bounded norm and $\widetilde{T} f^{n} \mid K(\tilde{m}) \equiv 0$ for some $n$. $\widetilde{T} f(K(\tilde{m})) \subset K(\tilde{f} m)$. Now we may apply the Oseledec theorem to the subbundle $V=\bigcup_{\tilde{m} \in \tilde{M}} V(\tilde{m})$ of $\widetilde{T M}$. For every orbit we have a splitting $E_{1}\left(m_{i}\right) \oplus E_{2}\left(m_{i}\right) \oplus \cdots \oplus E_{k}\left(m_{i}\right) \oplus G_{\infty}(\tilde{m})$ and exponents $\lambda_{1} \geq \cdots \geq \lambda_{k}$. Now we can make an arbitrarily small measurable perturbation to make $\tilde{T} f(K(\tilde{m})) \rightarrow K(\tilde{f} m)$ isomorphisms thus producing an exponent for $\tilde{T} f \mid K(\tilde{m}), \lambda_{k+1}$ less than $\lambda_{k}$. Applying Mañé Theorems A and $\mathrm{C}$ in the nonsingular case now will complete the argument for $(\mathrm{a})-(\mathrm{g})$.

As in [FHY] we want to let

$$
\begin{aligned}
& \bar{A}_{\varepsilon}(\tilde{m})=\sup _{\substack{n \geq 0 \\
k \in \mathbf{Z}}}\left\|T_{\tilde{f}^{k}(\tilde{m})} \tilde{f}^{n} \mid E_{\tilde{f}^{k}(\tilde{m})}^{s}\right\| e^{-(\lambda-\varepsilon) n-\varepsilon|k|}, \\
& \tilde{A}_{\varepsilon}(\tilde{m})=\sup _{\substack{n \geq 0 \\
k \in \mathbf{Z}}}\left\|T_{\tilde{f}^{k}(\tilde{m})} \tilde{f}^{-n} \mid E_{\tilde{f}^{k}(\tilde{m})}^{u}\right\| e^{-(\lambda+\varepsilon) n-\varepsilon|k|}
\end{aligned}
$$

and, finally, $A_{\varepsilon}(\tilde{m})=\sup \left(\bar{A}_{\varepsilon}(\tilde{m}), \tilde{A}_{\varepsilon}(\tilde{m})\right)$. We only have to prove that $\bar{A}_{\varepsilon}(\tilde{m})$ and $\tilde{A}_{\varepsilon}(\tilde{m})$ are finite in a set of total probability 1 . 
Let $C_{\varepsilon}(\tilde{m})=\sup \left\|T \tilde{f}^{n} \tilde{m} \mid E_{\tilde{m}}^{s}\right\| e^{-(\lambda-\varepsilon) n}$. Since $\|T \tilde{f}\|$ is bounded, there is a constant $B>0$ such that $C_{\varepsilon}(\tilde{m}) \leq B C_{\varepsilon}(\tilde{f}(\tilde{m}))$. Thus for $\phi(\tilde{m})=\log C_{\varepsilon}(\tilde{m})-$ $\log C_{\varepsilon}(\tilde{f} \tilde{m})$ we have $\phi(\tilde{m}) \leq \log B . \log C_{\varepsilon}(\tilde{m})$ is measurable, by Lemma III.8 of Mañé for any ergodic measure $\mu$

$$
\lim _{k \rightarrow \pm \infty} \frac{1}{|k|} \sum \phi\left(\tilde{f}^{k} \tilde{m}\right) \rightarrow \int \phi d \mu=0
$$

This establishes that $\tilde{A}_{\varepsilon}(\tilde{m})$ is finite on a total probability set.

Now $D_{\varepsilon}(\tilde{m})=\sup _{n \geq 0}\left\|T \tilde{f}^{-n} \mid E_{\tilde{m}}^{u}\right\| e^{-(\lambda+\varepsilon) n}$ defines a measurable function by [M1] since we are in the injective case. There is a constant $B$ such that $B D_{\varepsilon}(\tilde{f}(\tilde{m})) \geq D_{\varepsilon}(\tilde{m})$, since $\left\|T \tilde{f}^{-n}\left|E_{\tilde{m}}^{u}\|\leq\| T f^{-(n+1)}\right| E_{\tilde{f} \tilde{m}}^{u}\right\|\left\|T f \mid E_{\tilde{m}}^{u}\right\|$ and the second factor is bounded. Thus $\log B \geq \log D_{\varepsilon}(\tilde{m})-\log D_{\varepsilon}(\tilde{f}(\tilde{m}))$ and we proceed as above.

As an immediate corollary we have

5.3. Corollary. Let $f: M \rightarrow M$ be a $C^{1+\beta}$ endomorphism and let $\mu$ be an invariant measure for $f$ with no zero exponents. Then for almost all full orbits of $f$ there are stable and unstable disc families which are Borel, vary subexponentially along orbits and are invariant.

Proof. Let $\nu$ be an invariant measure on $\widetilde{M}$ which projects to $\mu$. It has the same exponent; see [RS] for example.

\section{HighER DIFFERENTIABILITY}

In this section we generalize the $C^{r}$-Section Theorem of [HPS]; see also [S]. Then we read off the corresponding-and sharp- $C^{r}$-Pesin Stable Manifold Theorem for $r>1$.

We assume that $H$ is a Borel subset of our manifold $M$ and that $h: H \rightarrow H$ is a Borel bijection. Let $\mathscr{B}_{\varepsilon}$ be the set of $\varepsilon$-slowly varying Borel functions $B: H \rightarrow(0, \infty)$. That is, $1 /(1+\varepsilon) \leq B(h p) / B(p) \leq 1+\varepsilon$ for all $p \in H$. It is easy to see that $\mathscr{B}_{\varepsilon}$ is closed under sums, positive multiples, convex combinations, and pointwise convergence. Besides, if $B, B^{\prime} \in \mathscr{B}_{\varepsilon}$ then their product belongs to $\mathscr{B}_{\varepsilon} \bullet \mathscr{B}_{\varepsilon}$, which we denote by $\mathscr{B}_{\varepsilon}^{2}$. Clearly, $\mathscr{B}_{\varepsilon}^{2}=\mathscr{B}_{\varepsilon^{\prime}}$ where $1+\varepsilon^{\prime}=(1+\varepsilon)^{2}$.

It will be useful to consider functions with growth rates $\mathscr{B}_{\varepsilon}^{\text {(r) }}$ where the exponent $\rho$ depends on $r$, the degree of differentiability of the dynamics, and $\mu(r)=3^{3^{r}}$. A handy property of $\rho$, whose verification is left to the reader, is

$$
(t+4+t \mu(t)) \rho(t) \leq \rho(t+1), \quad t \geq 0 .
$$

Definition. The $C^{r}$-size of a $C^{r}$ function $f(x)$ is $|f|_{C^{r}}=|f|_{0}+|f|_{r}$ where $|f|_{0}=\sup |f(x)|$ and $|f|_{r}=\sup \left\{\left\|D^{i} f\right\|: 1 \leq i \leq r\right\}$. If $f=f(p, x)$ for $p \in H$ then we say that $f$ is of class $C_{\varepsilon}^{r}$ provided that its $C^{r}$-size is $\mathscr{B}_{\varepsilon}$ bounded. That is, for some $B \in \mathscr{B}_{\varepsilon}$ and all $p \in H,|f(p, \cdot)|_{C^{r}} \leq B(p)$. The domain of definition of $f(p, \cdot)$ may depend on $p$. 
Let $X, Y$ be fixed Banach spaces and consider $E=H \times X \times Y$ as a trivial $Y$-bundle over $H \times X$. Let $r, R \in \mathscr{B}_{\varepsilon}$ be given. Call

$$
D=\{(p, x, y) \in E:|x| \leq r(p) \text { and }|y| \leq R(p)\} .
$$

We think of $D$ as a "trivial disc bundle of varying radius". Its base space is the "varying product" $H \times{ }_{r} X=\{(p, x) \in H \times X:|x| \leq r(p)\}$.

A section of $D$ is a map $\sigma: H \times_{r} X \rightarrow D$ of the form

$$
\sigma(p, x)=(p, x, s(p, x)) .
$$

The function $s$ is called the fiber component of $\sigma$. Under the norm

$$
|\sigma|=\sup \left\{|s(p, x)| / R(p):(p, x) \in H \times{ }_{r} X\right\},
$$

$\operatorname{Sec}(D)$ is complete.

Let us consider a fiber contraction of $D$, i.e., a map $F: D \rightarrow E$ making the diagram

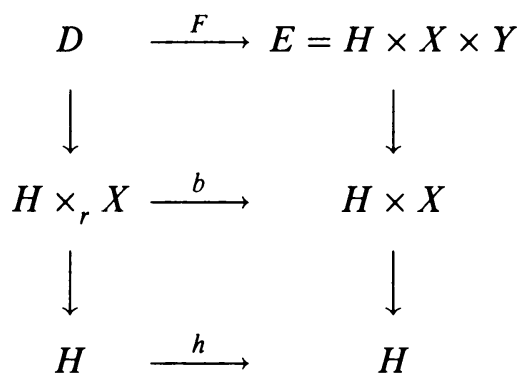

commute and satisfying

(a) $F(D) \cap\left(H \times{ }_{r} X \times Y\right) \subset D$ and $k=\sup \operatorname{Lip}(F(p, x, \cdot))<1$.

(b) $b$ embeds each $p \times X(r(p))$ onto a subset of $h p \times X$ which contains $h p \times X(r(h p))$.

By "Lip" we refer to the Lipschitz constant. The constant $k<1$ is called the fiber constant of $F$. Correspondingly, the base constant of $F$ is

$$
\alpha=\inf \left\{\frac{\left|b(p, x)-b\left(p, x^{\prime}\right)\right|}{\left|x-x^{\prime}\right|}:(p, x),\left(p, x^{\prime}\right) \in H \times_{r} X\right\} .
$$

The fiber constant measures how sharply $F$ contracts the fibers and the base constant measures how sharply $b$ expands the base. We do not assume that $\alpha>1$.

Definition. $F$ is an $r$-fiber contraction, $r \geq 0$, if it is $C_{\varepsilon}^{r}$ and

$$
k \alpha^{-s}(1+\varepsilon)^{\mu(r)}<1 \text { for all } s, 0 \leq s \leq r,
$$

$\rho(r)$ being the exponent $3^{3^{r}}$ referred to above. For any $r \geq 0$, this implies that $k(1+\varepsilon)<1$. Note that if $\varepsilon=0$ then the fibers of $D$ are uniformly bounded and $F$ is a uniform $r$-fiber contraction in the sense of [HPS, p. 80 and $\mathrm{S}]$. 
We see that $F$ defines a natural map $F_{\#}: \operatorname{Sec}(D) \rightarrow \operatorname{Sec}(D)$ by

$$
F_{\#}(\sigma)=\left.F \circ \sigma \circ b^{-1}\right|_{H \times, X}
$$

Equivalently, image $\left(F_{\#} \sigma\right)=F(\operatorname{image}(\sigma)) \cap D$. We call $F_{\#}$ the graph transform. If $F_{\#}(\sigma)=\sigma$ then we say that $\sigma$ is an $F$-invariant section.

The following result is the key to $\S 6$. It is more general than the $C^{r}$-Section Theorem of [HPS] in that the bundle where $F$ is defined has unbounded fibers and the derivatives of its fiber component are unbounded as well. It is less general in that the uniform assumption $k \alpha^{-r}<1$ replaces the pointwise assumption $\sup _{x} k_{x} \alpha_{x}^{-r}<1$.

6.1. $C_{\varepsilon}^{r}$-Section Theorem. An $r$-fiber contraction has a unique invariant section $\sigma_{F}$ and it is of class $C_{\delta}^{r}$ where $1+\delta=(1+\varepsilon)^{\mu(r)}$.

Remarks. The fiber contraction $F$ in 6.1 is once less differentiable than the diffeomorphism $f$ in $\S 3$. See 6.2. Finer estimates would let $\rho$ be replaced by the smaller exponent $2^{2^{r-1}}-1$.

It will be useful to understand some of the higher order properties of matrix inversion. Recall that Inv: $\mathscr{A} \supset$ is defined as $\operatorname{Inv}(A)=A^{-1}$ where $A \in \mathscr{A}$ and $\mathscr{A}$ is the set of invertible operators on some Banach space $E$. It is well known that Inv is smooth-in fact, analytic. Let us observe that

$$
\left(D^{s} \text { Inv }\right)_{A}\left(B_{1}, \ldots, B_{s}\right)=(-1)^{s} \sum_{\rho} A^{-1} B_{\rho(1)} A^{-1} B_{\rho(2)} A^{-1} \cdots B_{\rho(s)} A^{-1}
$$

where $\rho$ ranges over all permutations of $\{1, \ldots, s\}$ and $B_{1}, \ldots, B_{s}$ are operators on $E$. For $(D \operatorname{Inv})_{A}(B)=-A^{-1} B A^{-1}$ and if the above formula is true at stage $s$ then by Leibniz' Rule

$$
\begin{aligned}
D\left(D^{s} \text { Inv }\right)_{A}( & \left.B_{1}, \ldots, B_{s+1}\right) \\
=( & -1)^{s} \sum_{\rho}(-1) A^{-1} B_{s+1} A^{-1} B_{\rho(1)} A^{-1} \cdots B_{\rho(s)} A^{-1} \\
& +(-1) A^{-1} B_{\rho(1)} A^{-1} B_{s+1} A^{-1} \cdots B_{\rho(s)} A^{-1} \\
& +\cdots+(-1) A^{-1} B_{\rho(1)} A^{-1} \cdots B_{\rho(s)} A^{-1} B_{s+1} A^{-1}
\end{aligned}
$$

which is exactly the formula predicted at the $s+1$ stage. In the proof of 6.1 we use this formula to say that $D^{s}$ Inv is uniformly bounded on the set $\mathscr{A}(\mu)$ of $A$ with $\left\|A^{-1}\right\| \leq \mu$. In 6.3 we use

$$
D^{s} \text { Inv is uniformly Lipschitz on } \mathscr{A}(\mu) \text {. }
$$

It is interesting that (3) does not follow from the Mean Value Theorem, since $\mathscr{A}(\mu)$ is not convex. To verify (3) one "detelescopes" the difference $A^{-1} B_{\rho(1)}$ $A^{-1} \cdots B_{\rho(s)} A^{-1}-A^{\prime-1} B_{\rho(1)} A^{\prime-1} \cdots B_{\rho(s)} A^{\prime-1}$ into $s+1$ terms with a factor of $A^{-1}-A^{\prime-1}$ in place of successive $A^{-1}$ 's. Then one estimates this factor as 
$\left\|A^{-1}-A^{\prime-1}\right\|=\left\|A^{-1} A^{\prime} A^{\prime-1}-A^{-1} A A^{\prime-1}\right\| \leq \mu^{2}\left\|A-A^{\prime}\right\|$ and gets (3). In fact, $\operatorname{Lip}\left(D^{s}\left(\left.\operatorname{Inv}\right|_{\mathscr{A}(\mu)}\right)\right) \leq(s+1) ! \mu^{s+2}$.

It will also be necessary to use the Higher Order Chain Rule. For the composition of $C^{r}$ functions the HOCR says

$$
D^{r}(f \circ g)_{x}\left(v_{1}, \ldots, v_{r}\right)=\sum_{\gamma}\left(D^{t} f\right)_{g x}\left(D^{\gamma} g\right)_{x}\left(v_{\gamma}\right)
$$

where the multi-index $\gamma$ ranges over all partitions of $\{1, \ldots, r\}$ into disjoint nonempty subsets $\gamma: \gamma_{1} \sqcup \cdots \sqcup \gamma_{t}=\{1, \ldots, r\}$, and $v_{\gamma}$ means $\left(v_{1}, \ldots, v_{r}\right)$ arranged into blocks $\left(v_{\gamma_{1}}, \ldots, v_{\gamma_{t}}\right)$ where $v_{\gamma_{i}}$ is the block of vectors $v_{j}$ with $j \in \gamma_{i}$. The ordering of the $v_{j}$ inside a given block is irrelevant by the symmetry of higher derivatives. Likewise $D^{\gamma} g$ is the $t$-tuple of multilinear maps $\left(D^{\left|\gamma_{1}\right|} g, \ldots, D^{\left|\gamma_{t}\right|} g\right)$ and its ordering does not matter because $D^{t} f$ is symmetric. Note the welcome absence of numerical coefficients in the formula; we have not collected equal terms. The sum has $\leq r^{r}$ terms and so

$$
|f \circ g|_{r} \leq r^{r}|f|_{r}|g|_{r}^{r} \text {. }
$$

In the same way, if $Q_{z}$ and $R_{z}$ are composable linear operators depending $C^{t}$ on $z$ then by the Higher Order Leibniz Rule,

$$
D^{t}\left(Q_{z} \circ R_{z}\right)=\sum_{i=0}^{t}\left(\begin{array}{l}
t \\
i
\end{array}\right)\left(D^{t-i} Q_{z}\right) \circ\left(D^{i} R_{z}\right)
$$

Thus,

$$
\left|Q_{z} \circ R_{z}\right|_{C^{\prime}} \leq 2^{t}\left|Q_{z}\right|_{C^{\prime}}\left|R_{z}\right|_{C^{\prime}} .
$$

Proof of 6.1. Let $r=0$. Since $k(1+\varepsilon)<1, F_{\#}$ contracts the complete metric space $\operatorname{Sec}(D)$ into itself. The subset of continuous sections is closed and $F$ invariant, so the unique fixed point $\sigma_{F}$ of $F_{\#}$ is continuous. Since $\sigma_{F}$ covers the identity map on $H \times{ }_{r} X$ and $D$ has $\mathscr{B}_{\varepsilon}$-bounded fibers, $\sigma_{F}$ is of class $C_{\varepsilon}^{0}$. But for $r=0,1+\delta=(1+\varepsilon)^{3} \geq 1+\varepsilon$, and so $\sigma_{F}$ is of class $C_{\delta}^{0}$ as claimed.

Let $r=1$. Consider the action of $F$ on prospective tangent planes to the image of $\sigma_{F}$. A plane at $z$ is sent by $(D F)_{z}$ to a plane at $F(z)$. Since $\sigma_{F}$ is a section, the planes we consider will be graphs of linear maps $P: X \rightarrow Y$; i.e., $P \in L(X, Y)$. We write the derivative of $F$ at $z$ as the matrix

$$
(D F)_{z}=\left(\begin{array}{ll}
A_{z} & B_{z} \\
C_{z} & K_{z}
\end{array}\right), \quad \begin{array}{ll}
A_{z}: X \rightarrow X, & B_{z}: Y \rightarrow X, \\
C_{z}: X \rightarrow Y, & K_{z}: Y \rightarrow Y
\end{array}
$$

with respect to the $X$ and $Y$ variables. Thus,

$$
(D F)_{z}\left(\begin{array}{c}
\text { Id } \\
P
\end{array}\right)=\left(\begin{array}{c}
A_{z}+B_{z} P \\
C_{z}+K_{z} P
\end{array}\right) \text {. }
$$

Since $F$ preserves fibers of $D, B_{z} \equiv 0$ and since $b$ is an embedding, $A_{z}$ is invertible. Thus, the natural action of $F$ on $P \in L(X, Y)$, considered as a 
plane at $z \in D$, is $P F: P \mapsto\left(C_{z}+K_{z} P\right) \circ A_{z}^{-1}$. In terms of bundles,

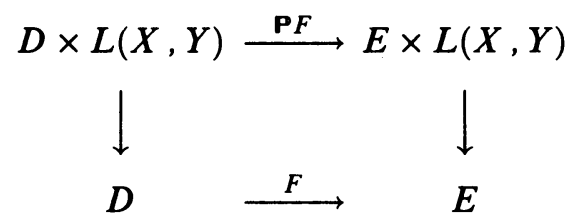

commutes and $P F$ is affine on fibers. It is $C^{r-1}$ and contracts fibers more sharply than the constant $k \alpha^{-1}<1$, because the minimum expansion of $A$ is at least $\alpha$ and $\left\|K_{z}\right\|<k$.

We cannot immediately assert that $P F$ has an invariant section because $F$ is not an appropriate base map. Being part of $(D F)_{z}, C_{z}$ is $\mathscr{B}$-bounded. Choose $B \in \mathscr{B}_{\varepsilon}$ and a large constant $c$ such that

$$
\begin{gathered}
\left\|C_{z}\right\| \leq B(p), \quad z \in D, \\
(1+\varepsilon)(1+c k) \alpha^{-1}<c .
\end{gathered}
$$

Consider the trivial disc bundle (of varying radius)

$$
L=\left\{(p, x, P) \in H \times{ }_{r} X \times L(X, Y):\|P\| \leq c B(p)\right\} .
$$

Like $D, L$ has $\mathscr{B}_{\varepsilon}$-bounded fibers. Define $L F$ on $L$ by

$$
\mathbf{L} F(p, x, P)=(b(p, x), \mathbf{P} F(z, P)),
$$

where $z=\sigma_{F}(p, x)$. Thus, $\mathbf{L F}=b \times\left(\mathbf{P} F \circ \sigma_{F}\right)$. By choice of $c$ in (7),

$$
\|\mathbf{P F}(z, P)\| \leq[B(p)+k c B(p)] \alpha^{-1} \leq(1+\varepsilon) B(h p)(1+c k) \leq c B(h p),
$$

whenever $(p, x, P) \in L$ and $z=\sigma_{F}(p, x)$. Also, the fiber constant $k \alpha^{-1}$ of LF obeys $\left(k \alpha^{-1}\right)(1+\varepsilon)^{\mu(0)}<1$ by (2) with $r=1=s$. Thus, $\mathbf{L} F$ is a 0 -fiber contraction of $L$,

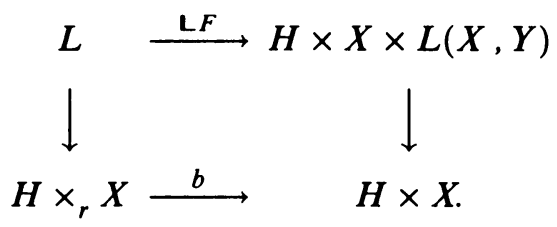

Let $\tau_{F}$ be its unique invariant section and let $t_{F}$ be the fiber component of $\tau_{F}$. We claim that $t_{F}$ is the derivative of $s_{F}$, the fiber component of $\sigma_{F}$,

$$
\frac{\partial s_{F}(p, x)}{\partial x}=t_{F}(p, x)
$$

This implies that $\sigma_{F}$ is $C^{l}$.

We follow the same path as in [HPS]. Consider the set $\operatorname{Sec}^{\prime}(D)$ of sections of $D$ whose slope over $p \times X(r(p))$ is $\leq c B(p)$. It is closed in $\operatorname{Sec}(D)$. For the slope of a section over $p \times X(r(p))$ is just the Lipschitz constant of its fiber component. We observe that $\operatorname{Sec}^{\prime}(D)$ is carried into itself by $F_{\#}$. For $D F$ 
carries any plane of slope $\leq c B(p)$ at $z$ onto one of slope $\leq c B(h p)$ at $F(z)$, and therefore $F$ never increases the slopes of secants from being $\leq c B(p)$ to being $>c B(h p)$. Hence, the unique fixed point $\sigma_{F}$ of $F_{\#}$ lies in $\operatorname{Sec}^{\prime}(D)$.

Next we consider two local sections $\sigma, \sigma^{\prime}$ of $D$ which agree at the point $\left(p, x_{0}\right)$. We assume they have slope $\leq c B(p)$. Under $F_{\#}, \sigma$ and $\sigma^{\prime}$ become local sections which agree at $b\left(p, x_{0}\right)=\left(h p, \xi_{0}\right)$. Let $\Delta_{x_{0}}$ be the Lipschitz constant of $s-s^{\prime}$ at $\left(p, x_{0}\right)$ where $s, s^{\prime}$ are the fiber components of $\sigma, \sigma^{\prime}$. That is,

$$
\Delta_{x_{0}}=\Delta_{x_{0}}\left(\sigma, \sigma^{\prime}\right)=\limsup _{x \rightarrow x_{0}} \frac{\left|s(p, x)-s^{\prime}(p, x)\right|}{\left|x-x_{0}\right|} .
$$

Let $\Delta_{\xi_{0}}$ be the Lipschitz constant of the difference of the fiber components of $F_{\#} \sigma$ and $F_{\#} \sigma^{\prime}$ at $\left(h p, \xi_{0}\right)$. Compare them:

$$
\begin{aligned}
\Delta_{\xi_{0}} & =\limsup _{\xi \rightarrow \xi_{0}} \frac{\left|g\left(\sigma\left(b^{-1} \xi\right)\right)-g\left(\sigma^{\prime}\left(b^{-1} \xi\right)\right)\right|}{\left|\xi-\xi_{0}\right|} \\
& \leq \limsup _{x \rightarrow x_{0}} k \frac{\left|s(p, x)-s^{\prime}(p, x)\right|}{\left|x-x_{0}\right|} \frac{\left|x-x_{0}\right|}{\left|\xi-\xi_{0}\right|} \leq k \Delta_{x_{0}} \alpha^{-1},
\end{aligned}
$$

where $b(p, x)=(h p, \xi)$ and $g$ is the fiber component of $F$. Thus,

$$
\Delta_{\xi_{0}} \leq k \alpha^{-1} \Delta_{x_{0}} .
$$

For any $z_{0}=\sigma_{F}\left(p, x_{0}\right)$, let $\lambda_{z_{0}}$ be a local section of $D$ defined in a neighborhood of $x_{0}$ such that the image of $\lambda_{z_{0}}$ is an affine plane $\Lambda_{z_{0}}$ parallel to the graph of $t_{F}\left(p, x_{0}\right)$ and passing through $z_{0}$. See Figure 11.

Call

$$
\Delta(p)=\sup \left\{\Delta_{x_{0}}\left(\lambda_{z_{0}}, \sigma_{F}\right): x_{0} \in X(r(p))\right\},
$$

$\Delta_{x_{0}}\left(\lambda_{z_{0}}, \sigma_{F}\right)$ being the Lipschitz constant of $\lambda_{z_{0}}-\sigma_{F}$ at $x_{0}$.

Since $\tau_{F}$ is $\mathscr{B}_{\varepsilon}$-bounded and $\sigma_{F}$ has $\mathscr{B}_{\varepsilon}$-bounded slope, $\Delta(p)$ is a $\mathscr{B}_{\varepsilon}$ bounded function. By naturality of $F_{\#}, F_{\#}\left(\lambda_{z_{0}}\right)$ is a $C^{1}$-local section such that

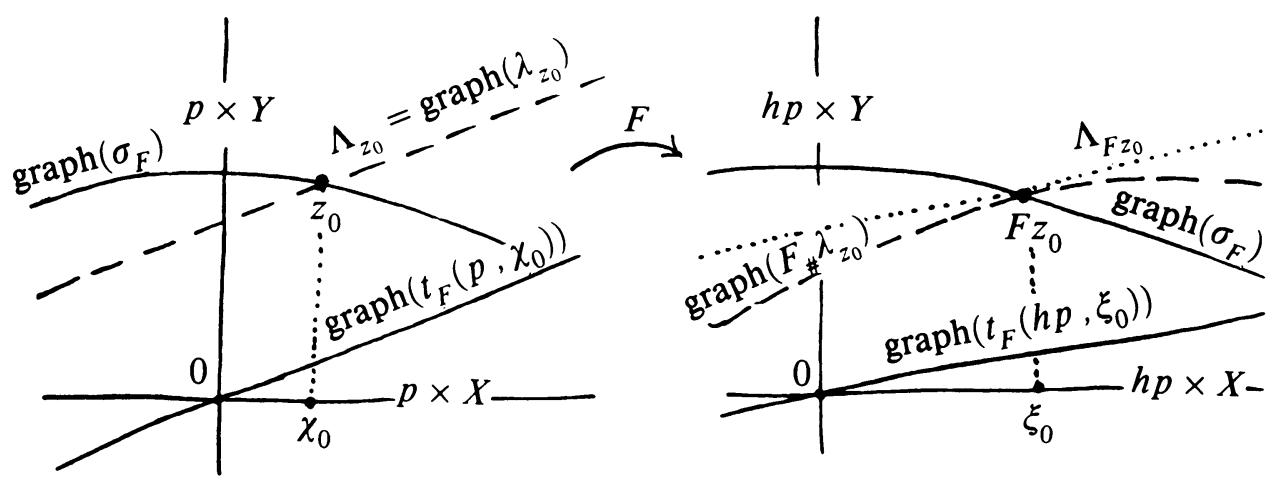

Figure 11. The effect of $F$ on $\lambda_{z_{0}}$ and $\sigma_{F}$ 
the tangent plane to image $\left(F_{\#} \lambda_{z_{0}}\right)$ at $F z_{0}$ is $\Lambda_{F z_{0}}$. Thus,

$$
\Delta(h p) \leq k \alpha^{-1} \Delta(p),
$$

by (9). However, any $\mathscr{B}_{\varepsilon}$-bounded function obeying inequality (10) must vanish identically. For if $\Delta(p) \neq 0$ then $\Delta\left(h^{-n} p\right) \geq\left(k \alpha^{-1}\right)^{-n} \Delta(p)$ and this quantity grows too fast for $\Delta$ to lie in $\mathscr{B}_{\varepsilon}$. But $\Delta \equiv 0$ is exactly what (8) means. Hence $\sigma_{F}$ is $C^{1}$ and $D \sigma_{F}$ is $\mathscr{B}_{\varepsilon}$-bounded; i.e., $\sigma_{F}$ is of class $C_{\varepsilon}^{1}$. Since $\mu(1)=27$, $\varepsilon<\delta$, and $\sigma_{F}$ is also of class $C_{\delta}^{1}$, completing the proof of $6.1: r=1$.

Let $r \geq 2$. We are assuming that $F$ is an $r$-fiber contraction:

$$
k \alpha^{-s}(1+\varepsilon)^{\mu(r)}<1, \quad 0 \leq s \leq r,
$$

$k$ and $\alpha$ being the fiber and base constants of $F$. Consider the same tangent fiber contraction as above,

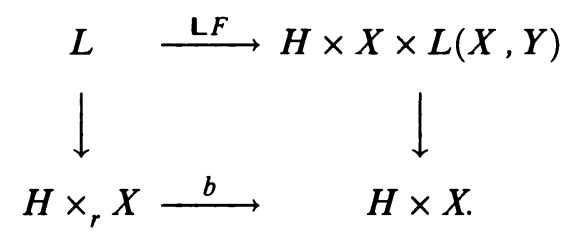

Its fiber constant is $k \alpha^{-1}$ and its base constant is $\alpha$. Define $\varepsilon^{\prime}$ by

$$
1+\varepsilon^{\prime}=(1+\varepsilon)^{r+1+(r-1) \mu(r-1)} \text {. }
$$

To apply 6.1: $r-1$ to $L F$ with $\varepsilon$ replaced by $\varepsilon^{\prime}$, we must show the $C^{r-1}$-size of $L F$ is $\mathscr{B}_{\varepsilon^{\prime}}$-bounded.

$$
\left(k \alpha^{-1}\right) \alpha^{-s}\left(1+\varepsilon^{\prime}\right)^{\mu(r-1)}<1 \text { for all } s, 0 \leq s \leq r-1 .
$$

It is immediate that (1) and (2) imply (12). To check (11) is harder. We estimate the $C^{r-1}$-size of $L F$ via the HOCR. Since $L F=b \times P F \circ \sigma_{F}$, and $b$ is of class $C_{\varepsilon}^{r}$, it is enough to worry about $P F \circ \sigma_{F}$. Since $\varepsilon<\varepsilon^{\prime}$, the fibers of $L$ are $\mathscr{B}_{\varepsilon^{\prime}}$-bounded, and we just need to estimate $\left|\mathrm{P} F \circ \sigma_{F}\right|_{r-1}$. By (4) we see that

$$
\left|\mathbf{P} F \circ \sigma_{F}\right|_{t} \leq t^{t}|\mathbf{P} F|_{t}\left|\sigma_{F}\right|_{t}^{t}, \quad 1 \leq t \leq r-1 .
$$

We must estimate $|\mathrm{P} F|_{t}$ where $\mathbf{P} F=\left(C_{z}+K_{z} P\right) \circ A_{z}^{-1}$. The HOCR applied to $A_{z}^{-1}=\operatorname{Inv} \circ\left(z \mapsto A_{z}\right)$ gives

$$
D^{i} A_{z}^{-1}=\sum_{\gamma} D^{s}(\operatorname{Inv})_{A_{z}} D^{\gamma} A_{z}
$$

where $\gamma$ ranges over partitions of $\{1, \ldots, i\}$. Now our initial remarks about inversion come into play. Since the norm of $A_{z}^{-1}$ is uniformly bounded, the higher derivatives of Inv are bounded and we see that $D^{i} A_{z}^{-1}$ is $\mathscr{B}_{\varepsilon}^{i}$-bounded. On the other hand, $D^{t-i}\left(C_{z}+K_{z} P\right)$ is $\mathscr{B}_{\varepsilon}^{2}$-bounded due to the facts that $F$ 
is of class $C_{\varepsilon}^{r}$ and $P$ is $\mathscr{B}_{\varepsilon}$-bounded. (Note that these estimates apply to derivatives involving $P$ as well as $z$.) Thus, by (5), $|\mathbf{P} F|_{t}$ is $\mathscr{B}_{\varepsilon}^{2+t}$-bounded. By induction we get to assume that

$$
\sigma_{F} \text { is of class } C_{\nu}^{t} \text { where } 1+\nu=(1+\varepsilon)^{\mathcal{R}(t)}, \quad 0 \leq t \leq r-1 .
$$

Thus, by (13) and (14), $|\mathbf{L} F|_{t}$ is $\mathscr{B}_{\varepsilon}{ }^{2+t /(t)}$-bounded, $0 \leq t \leq r-1$. Taking $t=r-1$ gives (11).

Now we can apply 6.1: $r-1$ to $L F$ and conclude that its unique invariant section $\sigma_{L F}$ is of class $C_{\delta^{\prime}}^{r-1}$ where $1+\delta^{\prime}=\left(1+\varepsilon^{\prime}\right)^{\mu(r-1)}<(1+\varepsilon)^{\mu(r)}=1+\delta$. But according to (8), $\sigma_{\mathrm{LF}}$ is just the tangent plane field to $\sigma_{F}$. Therefore, $\sigma_{F}$ is of class $C_{\delta}^{r}$ as claimed. Q.E.D.

6.2. Corollary. If the diffeomorphism $f: M \rightarrow M$ is of class $C^{r}, r$ being an integer $\geq 2$, then the stable and unstable manifolds of weak hyperbolic orbits are of class $C^{r}$.

Proof. We consider a $C^{r-1}$-fiber contraction $F$ which, roughly speaking, is the effect of $T f$ on prospective tangent planes to $W^{u}$. We show that $F$ satisfies the hypotheses of 6.1 and conclude that the tangent plane field on $W^{u}$ is $C^{r-1}-$ i.e., $W^{u}$ is $C^{r}$.

Instead of dealing with just one orbit, we may as well work with all orbits passing through a small hyperbolic block $P$. As in $\S 3$, let $\langle,\rangle^{*}$ be the adapted inner product over the saturate of $P$ and let $H$ be the disjoint union of the iterates $H=\sqcup f^{n}(P)$. Let $X=\mathbf{R}^{u}$ and $Y=\mathbf{R}^{s}$ with their standard inner products. Any two inner product spaces of the same dimension are isometric (this is why dealing with norms and Finslers alone is not good enough); let $i: H \times X \times Y \rightarrow E_{H}^{u} \oplus E_{H}^{s} \approx T_{H} M$ be an isometric isomorphism carrying $H \times X$ to $E_{H}^{u}$ and $H \times Y$ to $E_{H}^{s}$. (We identify $H \times X$ with $H \times X \times 0$, etc., and we assume that $i$ covers the identity on $H$.) Since $P$ is small, $E^{u}$ and $E^{s}$ are uniformly continuously trivial over it. For by 3.7 they extend to continuous trivial bundles on its closure. Thus, we may assume that $i$ is a homeomorphism of each $f^{n} P \times X \times Y$ onto $T_{f^{n} P} M$. (In $\S 3$, points in $H$ are called " $z$ "; here, they are denoted by " $p$ ", and " $z$ " refers to a point in $D$.)

As in $\S 3$, let $\bar{f}$ be the lift of $f$ to $T M$ via the smooth exponential. Using $i$, pull $\bar{f}$ back to a map $i^{*} \bar{f}$ defined on a neighborhood $D$ of the zero section in $H \times X \times Y$. That is,

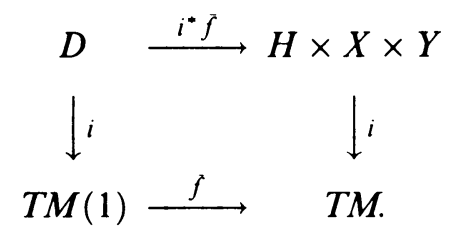

For notational simplicity we shall call $f=i^{*} \bar{f}$ and thus speak of $f$ as a map defined on $D$. As with $\bar{f}$, we write $f(z)=\left(f(p), f_{p}(x, y)\right)$ where $z=(p, x, y) \in D$. The norms we use on $D$ will be written || and \|\| , but 
they correspond isometrically to ||$^{*}$ and \|\|$^{*}$ in $T M$. In particular, if we express $D f$ as

$$
\left(D f_{p}\right)_{z}=\left(\begin{array}{ll}
A_{z} & B_{z} \\
C_{z} & K_{z}
\end{array}\right), \quad \begin{array}{ll}
A_{z}: X \rightarrow X, & B_{z}: Y \rightarrow X, \\
C_{z}: X \rightarrow Y, & K_{z}: Y \rightarrow Y
\end{array}
$$

then, uniformly over $p \in H, A_{0}$ is an expansion, $K_{0}$ is a contraction, and $C_{0}=B_{0}=0$. Choose constants $k$ and $\alpha$ such that $\sup _{p}\left\|K_{0}\right\|<k<1<\alpha<$ $\inf _{p} m\left(A_{0}\right)$. (Recall that the minimum norm, or conorm, of an operator $A$ is defined to be $m(A)=\inf \{|A v|:|v|=1\}$. For invertible operators, $m(A)=$ $\left\|A^{-1}\right\|^{-1}$.) Choose and fix an $\varepsilon>0$ so small that

$$
\left(k \alpha^{-1}\right) \alpha^{-s}(1+\varepsilon)^{\mu(r)}<1 \text { for all } s, 0 \leq s \leq r-1 .
$$

As in 6.1, $\mu(r)=3^{3^{r}}$. (Note that the existence of $\varepsilon$ follows from $k<\alpha$ and $k \alpha^{-r}<1$; we do not need to use $k<1<\alpha$, so the proof below can be modified to handle the nonhyperbolic case too. See 6.6.)

By 3.4 there exists a Borel radius function $r \in \mathscr{B}_{\varepsilon}$ such that $D$ contains $H \times{ }_{r}(X \times Y)$ and on this subset $f_{p}$ is uniformly $C^{1}$-approximated by its derivative $\left(D f_{p}\right)_{0}$. That is, $\left\|A_{z}-A_{0}\right\|$ is small, $\left\|B_{z}\right\|$ is small, $\left\|C_{z}\right\|$ is small, $\left\|K_{z}-K_{0}\right\|$ is small. Presently we say how small is small. For simplicity, assume $H \times{ }_{r}(X \times Y)$ equals $D$. In fact $f$ is so nearly fiber preserving that it has an invariant section $\sigma_{f}$

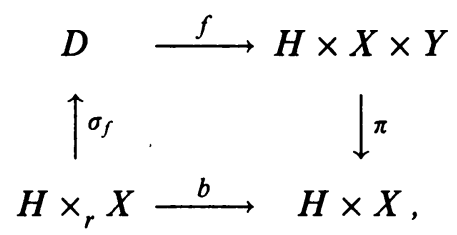

where $b=\pi \circ f \circ \sigma_{f}$ overflows the base. This section $\sigma_{f}$ is merely the local expression for $W^{u}-W_{r}^{u}(p)=i \circ \sigma_{f}(p \times X(r(p)))$, and $f$-invariance of $\sigma_{f}$ means that $f \circ \sigma_{f} \circ b^{-1}=\sigma_{f}$ on $H \times{ }_{r} X$. This follows from $\bar{f}$-invariance of $W^{u}$. In fact $\sigma_{f}$ is the unique section of slope $\leq 1$ which is $f$-invariant. By 3.8 we know that $\sigma_{f}$ is $C^{1}$ and $C^{1}$-bounded. We must prove that $\sigma_{f}$ is $C^{r}$. In fact, we assert

$$
\sigma_{f} \text { is of class } C_{\nu_{t}}^{t} \text { where } 1+\nu_{t}=(1+\varepsilon)^{\mu(t)}, 1 \leq t \leq r .
$$

Let $t=1$. Since $\sigma_{f}$ is $C^{1}$-bounded, it is certainly $C_{\nu_{1}}^{1}$.

Assume (16) is true up to and including some $t, 1 \leq t \leq r-1$. As in $\S 3$ we consider the $D f$-action, $\mathbf{P f}$, on prospective tangent planes to the image of $\sigma_{f}$. If $P \in L(X, Y)$ with $\|P\| \leq 1$ and $P$ represents such a plane at $z \in D$ then

$$
\mathbf{P} f(z, P)=\left(C_{z}+K_{z} P\right) \circ\left(A_{z}+B_{z} P\right)^{-1}
$$


represents its $D f$-image at $f(z)$. Clearly, $\mathbf{P} f$ is of class $C^{r-1}$. We define $F=b \times\left(\mathbf{P} f \circ \sigma_{f}\right)$, so $F(p, x, P)=\left(b(p, x), \mathbf{P} f\left(\sigma_{f}(p, x), P\right)\right)$ and

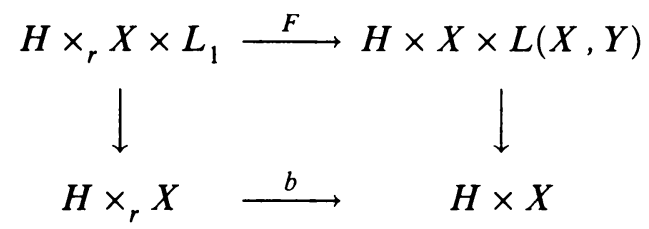

commutes. By $L_{1}$ we denote the unit ball in $L(X, Y)$. (In the notation of 6.1 this $F$ would be called $L f$.) We proceed to observe that $F$ is a fiber contraction. If $P \in L_{1}$ then

$$
\|\mathbf{P} f(z, P)\| \leq\left\{\left\|C_{z}\right\|+\left\|K_{z}\right\|\right\}\left\|\left(A_{z}+B_{z} P\right)^{-1}\right\| \leq k \alpha^{-1}<1 .
$$

For $\left\|C_{z}\right\|,\left\|B_{z}\right\|$ are small while $\left\|K_{z}\right\|\left\|A_{z}^{-1}\right\|$ is nearly equal to $\left\|K_{0}\right\|\left\|A_{0}^{-1}\right\|$, which is $<k \alpha^{-1}$. Similarly if $P, P^{\prime} \in L_{1}$ then

$$
\begin{aligned}
& \left\|\mathbf{P} f(z, P)-\mathbf{P} f\left(z, P^{\prime}\right)\right\| \leq\left\|K_{z}\right\|\left\|P-P^{\prime}\right\|\left\|\left(A_{z}+B_{z} P\right)^{-1}\right\| \\
& \quad+\left\{\left\|C_{z}\right\|+\left\|K_{z}\right\|\right\}\left\|\left(A_{z}+B_{z} P^{\prime}\right)^{-1}\right\|\left\|B_{z}\right\|\left\|P-P^{\prime}\right\|\left\|\left(A_{z}+B_{z} P\right)^{-1}\right\| \\
& \quad \leq k \alpha^{-1}\left\|P-P^{\prime}\right\| .
\end{aligned}
$$

Finally, $(D b)_{x}=A_{z}+B_{z} \circ D s_{f}$, where $z=\sigma_{f}(p, x)$ and $s_{f}$ is the fiber component of $\sigma_{f}$, and so the base constant of $F$ is $\geq \alpha$. These three requirements $(\|\mathbf{P} f(z, P)\|<1$, etc.) specify how small is small-i.e., how closely we require $\left(D f_{p}\right)_{0}$ to uniformly approximate $\left(D f_{p}\right)_{z}$.

We are going to estimate the $C^{t}$-size of $\mathbf{P} f \circ \sigma_{f}$. This is similar to the analysis in 6.1. First we observe that $|f|_{r}$ is $\mathscr{B}_{\varepsilon}$-bounded. For respecting the smooth inner product on $T M, D^{t} \bar{f}$ is uniformly bounded, and thus,

$$
\begin{aligned}
\left\|D^{t} \bar{f}_{p}\right\|^{*} & =\sup \left\{\left|D^{t} \bar{f}_{p}\left(v_{1}, \ldots, v_{t}\right)\right|^{*}:\left|v_{1}\right|^{*}=\cdots=\left|v_{t}\right|^{*}=1\right\} \\
& \leq B(f p) \sup \left\|D^{t} \bar{f}\right\|(\sqrt{2})^{t}
\end{aligned}
$$

follows from the comparison of $\langle$,$\rangle and \langle,\rangle^{*}, B$ being an appropriate element of $\mathscr{B}_{\varepsilon}$. The $C^{t}$ size of $f$ in $D$ is the same as the $C^{t}$ size of $\bar{f}$ respecting ||$^{*}$. Hence, $|f|_{r}$ is $\mathscr{B}_{\varepsilon}$-bounded.

As in 6.1, $\left|\left(A_{z}+B_{z} P\right)^{-1}\right|_{t}$ is $\mathscr{B}_{\varepsilon}^{t}$-bounded, $0 \leq t \leq r-1$. Clearly $\left|C_{z}+K_{z} P\right|_{t}$ is $\mathscr{B}_{\varepsilon}$-bounded since $\|P\| \leq 1$. Therefore, by $(5)$, the $C^{t}$-size of $\mathbf{P f}$ is $\mathscr{B}_{\varepsilon}^{t+1}$ bounded. But by (4), $\left|\mathbf{P} f \circ \sigma_{f}\right|_{t} \leq t^{t}|\mathbf{P} f|_{t}\left|\sigma_{f}\right|_{t}^{t}$, and so by $(16: t)$,

$$
\left|\mathbf{P} f \circ \sigma_{f}\right|_{t} \text { is } \mathscr{B}_{\varepsilon^{\prime}} \text {-bounded where } 1+\varepsilon^{\prime}=(1+\varepsilon)^{t+1+t /(t)} \text {. }
$$

By (1) and (15), $\left(k \alpha^{-1}\right) \alpha^{-s}\left(1+\varepsilon^{\prime}\right)^{\mu(t)}<1,0 \leq s \leq t$. Therefore, by (17), 6.1 can be applied to $F$ and so its unique invariant section $\sigma_{F}$ is of class $C_{\delta^{\prime}}^{t}$ where $1+\delta^{\prime}=\left(1+\varepsilon^{\prime}\right)^{\mu(t)}<(1+\varepsilon)^{\mu(t+1)}=1+\nu_{t+1}$. That is, $\sigma_{F}$ is of class $C_{\nu_{t+1}}^{t}$. But $\sigma_{F}$ is just the tangent plane field to $\sigma_{f}$ by uniqueness of the former and 
$D f$-invariance of the latter and so we have shown that $\sigma_{f}$ is $C_{\nu_{t+1}}^{t+1}$, completing the proof of $(16: t+1)$. Hence, $\sigma_{f}$-i.e., $W^{u}$-is $C^{r}$. Q.E.D.

Remark. We also showed that the $r$ th order tangent to $W^{u}(p)$ varies continuously as $p$ varies in the hyperbolic block $P$. For $\sigma_{F}$ is $C^{r-1}$ over each $f^{n} P$.

Finally, we show that $W^{u}$ is of class $C^{r+\beta}$ when $f$ is. To do so, we generalize 6.1. This involves dealing with Hölder functions that have unbounded domain and target-an awkward topic. If || is a norm and $\beta \in(0,1]$ is a constant, it will be convenient to write

$$
{ }^{\beta}|x|=\max \left\{|x|,|x|^{\beta}\right\}= \begin{cases}|x|^{\beta} & \text { if }|x| \leq 1 \\ |x| & \text { if }|x| \geq 1 .\end{cases}
$$

Let $g(x)$ be defined on a convex set and let $\beta \in(0,1]$ be given. Consider the two conditions:

(a) $\left|g(x)-g\left(x^{\prime}\right)\right| /\left|x-x^{\prime}\right|^{\beta}$ is bounded for all $x, x^{\prime}$ with $0<\left|x-x^{\prime}\right| \leq 1$.

(b) $\left.\left|g(x)-g\left(x^{\prime}\right)\right|\right|^{\beta}\left|x-x^{\prime}\right|$ is bounded for all $x, x^{\prime}$ with $\left|x-x^{\prime}\right| \neq 0$.

Clearly, (b) implies (a). Let us check the converse-in fact the constant in (b) is no more than twice that in (a). Assume $\left|x-x^{\prime}\right| \geq 1$. By convexity, $\left[x, x^{\prime}\right]$ can be divided into subsegments $x=x_{0}<\cdots<x_{n}=x^{\prime}$ such that $\left[x_{i-1}, x_{i}\right]$ has length 1 for $1 \leq i \leq n-1$, while $\left[x_{n-1}, x^{\prime}\right]$ has length $\leq 1$. Then

$$
\begin{aligned}
\left|g(x)-g\left(x^{\prime}\right)\right| & \leq\left|g\left(x_{0}\right)-g\left(x_{1}\right)\right|+\cdots+\left|g\left(x_{n-1}\right)-g\left(x^{\prime}\right)\right| \\
& \leq H\{1+\cdots+1\}+H\left|x_{n-1}-x^{\prime}\right|^{\beta} \\
& \leq H\left|x-x^{\prime}\right|+H\left|x-x^{\prime}\right|=2 H^{\beta}\left|x-x^{\prime}\right|
\end{aligned}
$$

where $H$ is the bound from (a), proving (b). On the other hand, if $\left|x-x^{\prime}\right| \leq 1$ then (b) is immediate. The bound in (a) or (b) is called a $\beta$-Hölder constant of $g$. If $g$ is of class $C^{r}$ and $D^{r} g$ has $\beta$-Hölder constant $H$ then the $C^{r+\beta}$-size of $g$ is $|g|_{0}+|g|_{r+\beta}$ where $|g|_{r+\beta}=|g|_{r}+H$.

Definition. If $g(p, x)$ is of class $C^{r+\beta}$ in $x, r \geq 0$, and if the $C^{r+\beta}$-size of $g(p, \cdot)$ is $\mathscr{B}_{\varepsilon}$-bounded then we say that $g$ is of class $C_{\varepsilon}^{r+\beta}$. If $F$ is a fiber contraction and it is of class $C_{\varepsilon}^{r+\beta}$ then we say that it is an $(r+\beta)$-fiber contraction provided that

$$
k \alpha^{-s}(1+\varepsilon)^{\mu(r+\beta)}<1 \text { for all } s, 0 \leq s \leq r+\beta .
$$

6.3. $C_{\varepsilon}^{r+\beta}$ Invariant Section Theorem. The invariant section of an $(r+\beta)$-fiber contraction is of class $C_{\delta}^{r+\beta}$ where $1+\delta=(1+\varepsilon)^{\mu(r+\beta)}$.

Proof. Let $\sigma_{F}=\mathrm{id} \times s_{F}$ be the invariant section of $F$ and write $F$ as $b \times g$. Define

$$
\mu=\max \left(\alpha^{-\beta}, \alpha^{-1}\right)= \begin{cases}\alpha^{-\beta} & \text { if } \alpha \geq 1, \\ \alpha^{-1} & \text { if } \alpha \leq 1 .\end{cases}
$$


(This $\mu$ is different from the $\mu$ in $\S \S 3$, 4.) Let $x_{1}=b^{-1}(p, x)$ and $x_{1}^{\prime}=$ $b^{-1}\left(p, x^{\prime}\right)$. Since $b$ expands more sharply than $\alpha$, we see that if $\left|x_{1}-x_{1}^{\prime}\right| \geq 1$ then

$$
{ }^{\beta}\left|x_{1}-x_{1}^{\prime} \dot{\mid}=\right| x_{1}-x_{1}^{\prime}\left|\leq \alpha^{-1}\right| x-x^{\prime}\left|\leq \mu^{\beta}\right| x-x^{\prime} \mid .
$$

On the other hand, if $\left|x_{1}-x_{1}^{\prime}\right| \leq 1$ then ${ }^{\beta}\left|x_{1}-x_{1}^{\prime}\right| \leq \alpha^{-\beta}\left|x-x^{\prime}\right|^{\beta} \leq \mu^{\beta}\left|x-x^{\prime}\right|$. In either case, $\operatorname{Lip}\left(b^{-1}\right) \leq \mu$ respecting ${ }^{\beta}||$ :

$$
{ }^{\beta}\left|x_{1}-x_{1}^{\prime}\right| \leq \mu^{\beta}\left|x-x^{\prime}\right| \text {. }
$$

Let $r=0$. As in [S, p. 46] we use $F$-invariance of $\sigma_{F}$ to estimate

$$
\Delta_{n}=\left|s_{F}\left(x_{n}\right)-s_{F}\left(x_{n}^{\prime}\right)\right|
$$

where $x_{n}=b^{-n}(p, x)$ and $x_{n}^{\prime}=b^{-n}\left(p, x^{\prime}\right)$. Let $H_{n}$ be the $\beta$-Hölder constant of $F$ over $h^{-n}(p)$. Then

$$
\begin{aligned}
\Delta_{0} & =\left|s_{F}(p, x)-s_{F}\left(p, x^{\prime}\right)\right|=\left|g\left(x_{1}, s_{F}\left(x_{1}\right)\right)-g\left(x_{1}^{\prime}, s_{F}\left(x_{1}^{\prime}\right)\right)\right| \\
& \leq\left|g\left(x_{1}, s_{F}\left(x_{1}\right)\right)-g\left(x_{1}, s_{F}\left(x_{1}^{\prime}\right)\right)\right|+\left|g\left(x_{1}, s_{F}\left(x_{1}^{\prime}\right)\right)-g\left(x_{1}^{\prime}, s_{F}\left(x_{1}^{\prime}\right)\right)\right| \\
& \leq k \Delta_{1}+H_{1}^{\beta}\left|x_{1}-x_{1}^{\prime}\right| \leq k \Delta_{1}+H_{1} \mu^{\beta}\left|x-x^{\prime}\right| \\
& \leq \cdots \leq k^{n} \Delta_{n}+\mu\left\{H_{1}+\cdots+H_{n}(k \mu)^{n-1}\right\}^{\beta}\left|x-x^{\prime}\right| .
\end{aligned}
$$

As $n \rightarrow \infty, k^{n} \Delta_{n} \rightarrow 0$ because the radius of $D$ is $\mathscr{B}_{\varepsilon}$-bounded and $(1+\varepsilon) k<1$ since $F$ is a 0 -fiber contraction. Similarly, since $H \in \mathscr{B}_{\varepsilon}$ and since $k \mu(1+\varepsilon)<$ 1 by (18), the sum in curly brackets converges,

$$
\sum_{n=1}^{\infty} H_{n}(k \mu)^{n-1} \leq \frac{H_{0}(1+\varepsilon)}{1-k \mu(1+\varepsilon)}
$$

Thus, if $c$ is the constant $(1+\varepsilon) /\{1-k \mu(1+\varepsilon)\}$ then

$$
\left|s_{F}(p, x)-s_{F}\left(p, x^{\prime}\right)\right|=\Delta_{0} \leq c H_{0}{ }^{\beta}\left|x-x^{\prime}\right|
$$

for all $x, x^{\prime} \in X(r(p))$. The same estimates show $\left|s_{F}\left(h^{-n} p, x\right)-s_{F}\left(h^{-n} p, x^{\prime}\right)\right|$ $\leq c H_{n}{ }^{\beta}\left|x-x^{\prime}\right|$ for all $x, x^{\prime} \in X\left(r\left(h^{-n} p\right)\right)$. Thus, $\sigma_{F}$ is of class $C_{\varepsilon}^{0+\beta}$ and 6.3 is proved for $r=0$.

Let $r \geq 1$. We know that $\sigma_{F}$ is $C^{r}$ and $D \sigma_{F}$ is found as the invariant section of the $(r-1)$-fiber contraction

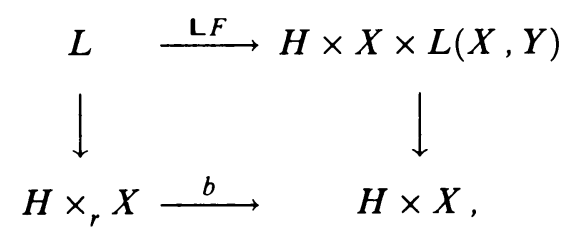

where $\mathrm{L} F=b \times \mathbf{P} F \circ \sigma_{F}$, and $\mathbf{P} F(z, P)=\left(C_{z}+K_{z} P\right) A_{z}^{-1}$. We want to apply 6.3: $r-1+\beta$ to $L F$, so we must estimate its $C^{r-1+\beta}$-size. To do so it is convenient to generalize the composition formulas (4) and (5) to the Hölder category. 
Suppose that $f$ and $g$ are $C^{r+\beta}$ functions defined on normed spaces, $r \geq 1$, and ${ }^{\beta} \operatorname{Lip}(g)$ refers to the Lipschitz constant respecting ${ }^{\beta}||$ :

$$
{ }^{\beta} \operatorname{Lip}(g)=\sup \frac{{ }^{\beta}\left|g(x)-g\left(x^{\prime}\right)\right|}{{ }^{\beta}\left|x-x^{\prime}\right|} .
$$

Then

$$
|f \circ g|_{r+\beta} \leq(r+1)^{r+1}|f|_{r+\beta}|g|_{r+\beta}^{r}\left\{1+{ }^{\beta} \operatorname{Lip}(g)\right\} .
$$

By (4) it suffices to worry about the $\beta$-Hölder constant of $D^{r}(f \circ g)$. Let $z=g(x)$ and $z^{\prime}=g\left(x^{\prime}\right)$. By the HOCR,

$$
\begin{aligned}
&\left\|D^{r}(f \circ g)_{x}-D^{r}(f \circ g)_{x^{\prime}}\right\|=\left\|\sum_{\gamma}\left(D^{t} f\right)_{z}\left(D^{\gamma} g\right)_{x}-\left(D^{t} f\right)_{z^{\prime}}\left(D^{\gamma} g\right)_{x^{\prime}}\right\| \\
& \leq \sum_{\gamma}\left\|\left(D^{t} f\right)_{z}\left(\left(D^{\left|\gamma_{1}\right|} g\right)_{x}-\left(D^{\left|\gamma_{1}\right|} g\right)_{x^{\prime}}, \ldots,\left(D^{\left|\gamma_{t}\right|} g\right)_{x}\right)\right\| \\
& \quad+\cdots+\sum_{\gamma}\left\|\left(D^{t} f\right)_{z}\left(\left(D^{\left|\gamma_{1}\right|} g\right)_{x^{\prime}}, \ldots,\left(D^{\left|\gamma_{t}\right|} g\right)_{x}-\left(D^{\left|\gamma_{t}\right|} g\right)_{x^{\prime}}\right)\right\| \\
& \quad+\sum_{\gamma}\left\|\left(D^{t} f\right)_{z}-\left(D^{t} f\right)_{z^{\prime}}\right\|\left\|\left(D^{\gamma} g\right)_{x^{\prime}}\right\| \\
& \leq r r^{r}|f|_{r}|g|_{r}^{r-1}|g|_{r+\beta}{ }^{\beta}\left|x-x^{\prime}\right|+r^{r}|f|_{r+\beta}|g|_{r}^{r \beta}\left|z-z^{\prime}\right| \\
& \leq\left(r^{r+1}+r^{r}\right)\left\{|f|_{r+\beta}|g|_{r+\beta}^{r}\right\}\left\{1+{ }^{\beta} \operatorname{Lip}(g)\right\}^{\beta}\left|x-x^{\prime}\right| .
\end{aligned}
$$

Thus, by (4)

$$
\begin{aligned}
|f \circ g|_{r+\beta} & =|f \circ g|_{r}+\beta \text {-Hölder constant of } D^{r}(f \circ g) \\
& \leq r^{r}|f|_{r}|g|_{r}^{r}+\left(r^{r+1}+r^{r}\right)\left\{|f|_{r+\beta}|g|_{r+\beta}^{r}\right\}\left\{1+{ }^{\beta} \operatorname{Lip}(g)\right\} \\
& \leq(r+1)^{r+1}|f|_{r+\beta}|g|_{r+\beta}^{r}\left\{1+{ }^{\beta} \operatorname{Lip}(g)\right\},
\end{aligned}
$$

which is (19). Similarly, if $Q_{z}$ and $R_{z}$ are composable linear operators that depend $C^{r+\beta}$ on $z$ then

$$
\left|Q_{z} \circ R_{z}\right|_{C^{r+\beta}} \leq 2^{r+1}\left|Q_{z}\right|_{C^{r+\beta}}\left|R_{z}\right|_{C^{r+\beta}}
$$

In our case (19) gives

$$
\left|\mathrm{P} F \circ \sigma_{F}\right|_{r-1+\beta} \leq r^{r}|\mathrm{P} F|_{r-1+\beta}\left|\sigma_{F}\right|_{r-1+\beta}^{r-1}\left(1+{ }^{\beta} \operatorname{Lip}\left(\sigma_{F}\right)\right) \text {. }
$$

To estimate $|\mathrm{P} F|_{r-1+\beta}$, we start with $A_{z}^{-1}$. Recall that $\mathscr{A}(\mu)$ is the set of invertible $A: X \rightarrow X$ such that $\left\|A^{-1}\right\| \leq \mu$. By (19),

$$
\left|A_{z}^{-1}\right|_{r-1+\beta} \leq r^{r}\left|\left(\left.\operatorname{Inv}\right|_{\mathscr{A}(\mu)}\right)\right|_{r-1+\beta}\left|A_{z}\right|_{r-1+\beta}^{r-1}\left\{1+{ }^{\beta} \operatorname{Lip}\left(A_{z}\right)\right\} \text {. }
$$

Now ${ }^{\beta} \operatorname{Lip}\left(A_{z}\right) \leq \max \left\{1, \operatorname{Lip}\left(A_{z}\right),\left(\operatorname{Lip}\left(A_{z}\right)^{\beta}\right\}\right.$. For ${ }^{\beta} \operatorname{Lip}\left(A_{z}\right)$ is the supremum of the ratios $\left.{ }^{\beta}\left\|A_{z}-A_{z^{\prime}}\right\|\right|^{\beta}\left|z-z^{\prime}\right|$. If all these ratios are $\leq 1$ then 
${ }^{\beta} \operatorname{Lip}\left(A_{z}\right) \leq 1$. On the other hand, if the numerator is $\geq 1$ then the ratio is $\leq \operatorname{Lip}\left(A_{z}\right)$. Finally, if the numerator is $\leq 1$ and the ratio is $\geq 1$ then it is $\leq \operatorname{Lip}\left(A_{z}\right)^{\beta}$. Thus,

$$
{ }^{\beta} \operatorname{Lip}\left(A_{z}\right) \text { is } \mathscr{B}_{\varepsilon} \text {-bounded. }
$$

Combining (3), (22), (23), we get

$$
\left|A_{z}^{-1}\right|_{C^{r-1+\beta}} \text { is } \mathscr{B}_{\varepsilon}^{r} \text {-bounded. }
$$

Next, $\left\|D^{j}\left(C_{z}+K_{z} P\right)-D^{j}\left(C_{z^{\prime}}-K_{z^{\prime}} P^{\prime}\right)\right\|$ is estimated as

$$
\begin{aligned}
& \left\|D^{j}\left(C_{z}+K_{z} P\right)-D^{j}\left(C_{z}+K_{z} P^{\prime}\right)\right\|+\left\|D^{j}\left(C_{z}+K_{z} P^{\prime}\right)-D^{j}\left(C_{z^{\prime}}+K_{z^{\prime}} P^{\prime}\right)\right\| \\
& \quad \leq|F|_{r}\left\|P-P^{\prime}\right\|+|F|_{r+\beta}\left(1+\left\|P^{\prime}\right\|\right)^{\beta}\left|z-z^{\prime}\right| \\
& \quad \leq|F|_{r+\beta}\left(1+\left\|P^{\prime}\right\|\right)\left\{{ }^{\beta}\left\|P-P^{\prime}\right\|+{ }^{\beta}\left|z-z^{\prime}\right|\right\},
\end{aligned}
$$

which shows that the $\beta$-Hölder constant of $D^{j}\left(C_{z}+K_{z} P\right)$ is $\mathscr{B}_{\varepsilon}^{2}$-bounded for $0 \leq j \leq r-1$. Thus,

$$
\left|C_{z}+K_{z} P\right|_{C^{r-1+\beta}} \text { is } \mathscr{B}_{\varepsilon}^{2} \text {-bounded. }
$$

Then, by (20), (24), (25),

$$
|\mathbf{P} F|_{r-1+\beta} \text { is } \mathscr{B}_{\varepsilon}^{2+r} \text {-bounded. }
$$

The next factor in (21) is $\left|\sigma_{F}\right|_{r-1+\beta}^{r-1}$. By induction we get to assume that $\sigma_{F}$ is of class $C_{\delta^{\prime}}^{r-1+\beta}$ where $1+\delta^{\prime}=(1+\varepsilon)^{\mu(r-1+\beta)}$. Thus,

$$
\left|\sigma_{F}\right|_{r-1+\beta}^{r-1} \text { is } \mathscr{B}_{\varepsilon}^{(r-1) \mu(r-1+\beta)} \text {-bounded. }
$$

It remains to estimate ${ }^{\beta} \operatorname{Lip}\left(\sigma_{F}\right)$. By 6.1 , we know that $\sigma_{F}$ is $C^{1}$ and since it is a section, its Lipschitz constant is $\geq 1$. Call $\Delta x=x-x^{\prime}$ and $\Delta \sigma_{F}=$ $\sigma_{F}(p, x)-\sigma_{F}\left(p, x^{\prime}\right)$. If $\left|\Delta \sigma_{F}\right| \leq 1$ then $|\Delta x| \leq 1$ and

$$
{ }^{\beta}\left|\Delta \sigma_{F}\right| /^{\beta}|\Delta x|=\left|\Delta \sigma_{F}\right|^{\beta} /|\Delta x|^{\beta} \leq \operatorname{Lip}\left(\sigma_{F}\right)^{\beta} \leq \operatorname{Lip}\left(\sigma_{F}\right) .
$$

On the other hand, if $\left|\Delta \sigma_{F}\right| \geq 1$ then

$$
\frac{{ }^{\beta}\left|\Delta \sigma_{F}\right|}{{ }^{\beta}|\Delta x|}=\frac{\left|\Delta \sigma_{F}\right|}{{ }^{\beta}|\Delta x|} \leq \frac{\left|\Delta \sigma_{F}\right|}{|\Delta x|} \leq \operatorname{Lip}\left(\sigma_{F}\right) .
$$

Consequently, ${ }^{\beta} \operatorname{Lip}\left(\sigma_{F}\right) \leq \operatorname{Lip}\left(\sigma_{F}\right)$. In the proof of 6.1 we showed that $\sigma_{F}$ is of class $C_{\varepsilon}^{1}$, so $\operatorname{Lip}\left(\sigma_{F}\right)$ is $\mathscr{B}_{\varepsilon}$-bounded; i.e.,

$$
{ }^{\beta} \operatorname{Lip}\left(\sigma_{F}\right) \text { is } \mathscr{B}_{\varepsilon} \text {-bounded. }
$$

Combining (21), (26), (27), and (28) shows that $\left|\mathbf{P} F \circ \sigma_{F}\right|_{r-1+\beta}$ is $\mathscr{B}_{\varepsilon}^{2+r+(r-1) \rho(r-1+\beta)+1}$-bounded. Since $b$ is of class $C_{\varepsilon}^{r+\beta}$ this implies that

$$
\mathbf{L} F=b \times \mathbf{P} F \circ \sigma_{F} \text { is of class } C_{\varepsilon^{\prime}}^{r-1+\beta},
$$


where $1+\varepsilon^{\prime}=(1+\varepsilon)^{3+r+(r-1) \mu(r-1+\beta)}$. By (18) and (1),

$$
\left(k \alpha^{-1}\right) \alpha^{-s}(1+\delta)^{\mu(r-1+\beta)}<1 \text { for all } s, 0 \leq s \leq r-1+\beta .
$$

But (29) and (30) say that $L F$ is an $(r-1+\beta)$-fiber contraction with $\varepsilon$ replaced by $\varepsilon^{\prime}$. By induction and (1), its unique invariant section $\sigma_{\mathrm{L} F}$ is of class $C_{\delta}^{r-1+\beta}$ where $1+\delta=(1+\varepsilon)^{\mu(r+\beta)}$. But $\sigma_{\mathrm{L} F}$ is just the tangent plane field to $\sigma_{F}$ and so $\sigma_{F}$ is of class $C_{\delta}^{r+\beta}$. Q.E.D.

6.4. Corollary. If the diffeomorphism $f: M \rightarrow M$ is of class $C^{r+\beta}, r+\beta>1$, then the stable and unstable manifolds of a weak hyperbolic orbit are also of class $C^{r+\beta}$. (Thus, $r=1$ and $\beta>0$ or $r \geq 2$ and $\beta \geq 0$.)

Proof. We revert to the notation of the proof of 6.2. Let $\sigma_{f}: H \times{ }_{r} X \rightarrow D$ be the unique $f$-invariant section of slope $\leq 1$. Its image is merely $W^{u}$ viewed in the adapted exponential coordinates. Choose and fix an $\varepsilon>0$ so small that

$$
\left(k \alpha^{-1}\right) \alpha^{-s}(1+\varepsilon)^{\mu(r+\beta)}<1 \text { for all } s, 0 \leq s \leq r+\beta .
$$

By 3.8 and 6.2 we know that $\sigma_{f}$ is $C_{\delta^{\prime}}^{r}$ where $1+\delta^{\prime}=(1+\varepsilon)^{\mu(r)}$. We want to show that $\sigma_{f}$ is $C_{\delta}^{r+\beta}$ where $1+\delta=(1+\varepsilon)^{\mu(r+\beta)}$. To do so it suffices to prove that the $(r-1)$-fiber contraction $F=b \times \mathbf{P} f \circ \sigma_{f}$ is actually an $(r-1+\beta)$-fiber contraction with $\varepsilon$ replaced by $\varepsilon^{\prime}$ and $1+\varepsilon^{\prime}=(1+\varepsilon)^{1+r+(r-1) \rho(r-1+\beta)}$. For then by (1) and 6.3 applied to $F$, we see that $\sigma_{F}$ is of class $C_{\delta}^{r-1+\beta}$. Since $\sigma_{F}$ is just the tangent plane field to $\sigma_{f}$, this means that $\sigma_{f}-i . e ., W^{u}$-is of class $C_{\delta}^{r+\beta}$ as claimed.

Estimating the $C^{r-1+\beta}$-size of $F$ is similar to the calculations in the proof of 6.3. First, the $C^{r+\beta}$-size of $f\left(=i^{*} f\right)$ is its $C^{r}$-size plus the $\beta$-Hölder constant of $D^{r} f$. The latter is found from

$$
\begin{aligned}
\frac{\left\|\left(D^{r} f\right)_{z}-\left(D^{r} f\right)_{z^{\prime}}\right\|^{*}}{{ }^{\beta}\left|z-z^{\prime}\right|^{*}} & \leq \frac{B(f p)\left\|\left(D^{r} \bar{f}\right)_{z}-\left(D^{r} \bar{f}\right)_{z^{\prime}}\right\|(\sqrt{2})^{r}}{\sqrt{1 / 2}^{\beta}\left|z-z^{\prime}\right|} \\
& \leq B(f p)(\sqrt{2})^{r+1}|\bar{f}|_{r+\beta}
\end{aligned}
$$

since $\left|z-z^{\prime}\right|^{*} \geq \sqrt{\frac{1}{2}}\left|z-z^{\prime}\right|$ implies

$$
\begin{aligned}
{ }^{\beta}\left|z-z^{\prime}\right|^{*} & =\max \left\{\left|z-z^{\prime}\right|^{* \beta},\left|z-z^{\prime}\right|^{*}\right\} \\
& \geq \max \left\{\left(\sqrt{\frac{1}{2}}\right)^{\beta}\left|z-z^{\prime}\right|^{\beta}, \sqrt{\frac{1}{2}}\left|z-z^{\prime}\right|\right\} \geq \sqrt{\frac{1}{2}}^{\beta}\left|z-z^{\prime}\right| .
\end{aligned}
$$

The function $B$ belongs to $\mathscr{B}_{\varepsilon}$. Thus, $|f|_{r+\beta}$ is $\mathscr{B}_{\varepsilon}$-bounded.

Second, we estimate $\left|\left(A_{z}+B_{z} P\right)^{-1}\right|_{r-1+\beta}$ just as we did in (22)-(24). It is $\mathscr{B}_{\varepsilon}^{r}$-bounded. Similarly, $C_{z}+K_{z} P$ has $\mathscr{B}_{\varepsilon}$-bounded $C^{r-1+\beta}$-size. By (20) we conclude that

$$
\text { Pf has } \mathscr{B}_{\varepsilon}^{1+r} \text {-bounded } C^{r-1+\beta} \text {-size. }
$$


By induction we get to assume that

$$
\left|\sigma_{f}\right|_{r-1+\beta} \text { is } \mathscr{B}_{\varepsilon}^{\mu(r-1+\beta)} \text {-bounded. }
$$

Finally, since $\sigma_{f}$ has slope $\leq 1,{ }^{\beta} \operatorname{Lip}\left(\sigma_{f}\right)$ is bounded. Combining (31), (32), and (33) via (18) we see that

$$
\left|\mathbf{P} f \circ \sigma_{f}\right|_{r-1+\beta} \text { is } \mathscr{B}_{\varepsilon}^{1+r+(r-1) \mu(r-1+\beta)} \text {-bounded. }
$$

Since $b=\pi \circ f \circ \sigma_{f}$ it follows from (18) and (33) that the $C^{r-1+\beta}$-size of $b$ is $\mathscr{B}_{\varepsilon}{ }^{1+(r-1)} \mu^{(r-1+\beta)}$-bounded. From (34),

$$
\begin{aligned}
& F=b \times \mathbf{P} f \circ \sigma_{f} \text { is of class } C_{\varepsilon^{\prime}}^{r-1+\beta} \\
& \text { where } 1+\varepsilon^{\prime}=(1+\varepsilon)^{1+r+(r-1) /(r-1+\beta)} .
\end{aligned}
$$

Moreover, from (31) and (1),

$$
\left(k \alpha^{-1}\right) \alpha^{-s}\left(1+\varepsilon^{\prime}\right)^{\rho(r-1+\beta)}<1 \text { for all } s, 0 \leq s \leq r-1+\beta .
$$

(35) and (36) mean that $F$ is an $(r-1+\beta)$-fiber contraction of $L$ with $\varepsilon$ replaced by $\varepsilon^{\prime}$. Hence, $\sigma_{F}$ is $C_{\delta}^{r-1+\beta}$ and $\sigma_{f}, W^{u}$ are $C_{\delta}^{r+\beta}$. Q.E.D.

The preceding analysis has assumed that the diffeomorphism $f$ is of class $C^{r}$ for a finite $r$. Now assume that $f$ is $C^{\infty}$. By 6.2 we know that the local unstable manifold $W_{p}^{u}(\rho)$ of a weak hyperbolic orbit is $C^{r}$ for any $r$, but the radius $\rho$ on which this is true may depend on $r$. By construction, $\rho$ varies slowly-so slowly that $\sqcup f^{-n}\left(W_{f^{n} p}(\rho)\right)$ is the global unstable manifold of $p$. See 3.8. Thus,

6.5. Corollary. If the diffeomorphism $f$ is of class $C^{\infty}$ then so are the unstable and stable manifolds along a weak hyperbolic orbit.

Finally, we analyze the pseudo-hyperbolic case. Let us assume that $H$ is an $f$-invariant Borel set of regular orbits whose growth rates $\lambda$ are confined to a fixed set of compact intervals $I_{l}<\cdots<I_{1}$. See 3.17. (In the hyperbolic case there are only two intervals, $I_{2}>1>I_{1}$.) According to 3.17 , tangent to $E_{i}=\bigoplus_{\lambda \in I_{i}} E^{\lambda}$ there exists a locally $f$-invariant family of discs $\mathscr{W}_{i}=\left\{W_{i}(p)\right\}$, $p \in H$.

6.6. Theorem. The discs $W_{i}(p)$ are of class $C^{r+\beta}$ and are $C^{r+\beta}$ Borel functions of $p \in H$ provided that the diffeomorphism $f$ is $C^{r+\beta}, r+\beta>1$, and $I_{i+1}<\left(I_{i}\right)^{s}<I_{i-1}$ for all $s, 1 \leq s \leq r+\beta$.

Proof. This requirement on the intervals is a "spacing condition" on the growth rates. By $(I)^{s}<I^{\prime}$ we mean that $t^{s}<t^{\prime}$ for all $t \in I, t^{\prime} \in I^{\prime}$. If $i=1$ or $i=l$ then only half the hypothesized inequality makes sense. Call $I_{i}=\left[a_{i}, b_{i}\right]$.

Fix an $i$. By assumption, for all $s, 1 \leq s \leq r+\beta$,

$$
\begin{aligned}
& I_{i+1}<\left(I_{i}\right)^{s} \Rightarrow b_{i+1}<a_{i}^{s}, \\
& \left(I_{i}\right)^{s}<I_{i-1} \Rightarrow b_{i}^{s}<a_{i-1} .
\end{aligned}
$$


Group the Lyapunov spaces $E_{j}$ as in 3.17,

$$
E_{i}^{p u}=E_{1} \oplus \cdots \oplus E_{i}, \quad G_{i}=E_{i+1} \oplus \cdots \oplus E_{l} .
$$

There is a locally $f$-invariant family of discs $\mathscr{W}_{i}^{p u}=\left\{W_{i}^{p u}\right\}$ tangent to $E_{i}^{p u}$. The tangent planes to these discs are located by the iteration of $T f$ on trial tangent planes-i.e., they are located as the $\mathbf{L} f$-invariant section $\sigma_{\mathbf{L} f}$ where $\mathbf{L} f=b \times\left(\mathbf{P} f \circ \sigma_{f}\right)$ as in 3.8, 6.2, and 6.4. The base map $b$ is essentially $f$ restricted to $W_{i}^{p u}$, and so the base constant of $\mathrm{L} f$ is the weakest expansion in $W_{i}^{p u}$, namely $\alpha=a_{i}$. The fiber constant of $\mathbf{L} f$ is $k=b_{i+1} a_{i}^{-1}$ because $b_{i+1}$ dominates the largest growth rate in $G_{i}$. By the hypothesis that $I_{i+1}<\left(I_{i}\right)^{s}$, we. have $k \alpha^{-s}<1$ for all $s, 0 \leq s \leq r-1+\beta$. By 6.3, $\sigma_{\mathrm{L} f}$ is $C^{r-1+\beta}$. Hence, $W_{i}^{p u}$ is $C^{r+\beta}$. The same analysis applies to $f^{-1}$ respecting the splitting $E_{i}^{p s} \oplus G_{i}^{\prime}$ where $E_{i}^{p s}=\bigoplus_{j \geq i} E_{j}$ and $G_{i}^{\prime}=\bigoplus_{j<i} E_{j}$ since $\left(I_{i}\right)^{s}<I_{i-1}$. Thus, $W_{i}^{p s}$ is $C^{r+\beta}$ and so is the locally $f$-invariant transverse intersection $W_{i}=$ $W_{i}^{p u} \cap W_{i}^{p s}$. Q.E.D.

6.7. Corollary. Over the set of regular orbits, let $E^{u}=\bigoplus_{\lambda>1} E^{\lambda}, E^{c}=\bigoplus_{\lambda=1} E^{\lambda}$, and $E^{s}=\bigoplus_{\lambda<1} E^{\lambda}$. If the diffeomorphism $f$ is of class $C^{r+\beta}, 1<r+\beta<\infty$, then there exist $C^{r+\beta}$ locally $f$-invariant center manifolds $W^{c}$ tangent to $E^{c}$.

Proof. We divide the set of regular orbits into saturates of pseudo-hyperbolic blocks so that on each, the growth rates of vectors in $E^{u}$ and $E^{s}$ are bounded away from 1 . Say they lie in $I_{u}, I_{s}$ and $I_{s}<1<I_{u}$. If $I_{c}$ is a sufficiently small interval around 1 then $I_{s}<\left(I_{c}\right)^{s}<I_{u}$ for all $s, 1 \leq s \leq r+\beta$ and the result follows from 6.6. Q.E.D.

\section{REFERENCES}

[A] D. V. Anosov, Geodesic flows on closed Riemann manifolds with negative curvature, Proc. Steklov Inst. Math., No. 90 (1967), English transl., Amer. Math. Soc., Providence, R.I., 1969.

[B'i] N. Bourbaki, Integration, Chapter 6, Hermann, Paris, 1959.

[B] M. Brown, The monotone union of open n-cells is an open n-cell, Proc. Amer. Math. Soc. 12 (1961), 812-814.

[FHY] A. Fathi, M. R. Herman, and J. C. Yoccoz, A proof of Pesin's stable manifold theorem, Geometric Dynamics (J. Palis, Jr., Ed.), Lecture Notes in Math., vol. 1007, Springer-Verlag, 1983, pp. 177-215.

[HPS] M. W. Hirsch, C. C. Pugh, and M. Shub, Invariant manifolds, Lecture Notes in Math., vol. 583, Springer-Verlag, 1977.

[K] A. Katok, Lyapunov exponents, entropy, and periodic points for diffeomorphism, Inst. Hautes Études Sci. Publ. Math. 51 (1980), 137-173.

[M1] R. Mañé, Lyapunov exponents and stable manifolds for compact transformations, Geometric Dynamics (J. Palis Jr., Ed.), Lecture Notes in Math., vol. 1007, Springer-Verlag, 1983, pp. 522-577.

[M2] __ Teoria ergodica, IMPA publication; English transl., Ergodic Theory and Differentiable Dynamics, Springer-Verlag, 1987. 
[M3] __, Quasi-Anosov diffeomorphisms and hyperbolic manifolds, Trans. Amer. Math. Soc. 229 (1977), 351-370.

[O] V. I. Oseledec, A multiplicative ergodic theorem, Lyapunov characteristic numbers for dynamical systems, Trudy Moskov. Mat. Obshch. 19 (1968); English transl., Trans. Moscow Math. Soc. 19 (1968), 197-231.

[P1] Ja. B. Pesin, Families of invariant manifolds corresponding to non-zero characteristic exponents, Izv. Akad. Nauk SSSR Ser. Mat. 40 (1976); English transl., Math. USSR-Izv. 10 (1976), 12611305.

[P2] _ Characteristic Lyapunov exponents and smooth ergodic theory, Uspekhi Mat. Nauk 32 (1977), 55-112; English transl., Russian Math. Surveys 32 (1977), 55-114.

[Pa] K. R. Parthasarathy, Probability measures on metric spaces, Academic Press, New York and London, 1967.

[P'n] K. Peterson, Ergodic theory, Cambridge Univ. Press, Cambridge, 1983.

[PS1] C. C. Pugh and M. Shub, Ergodicity of Anosov actions, Invent. Math. 15 (1972), 1-23.

[PS2] _ Differentiability and continuity of invariant manifolds, Non-linear Dynamics (R. G. Helleman, Ed.), New York Academy of Sciences, 1980, pp. 322-329.

[Pu] C. C. Pugh, The $C^{1+\alpha}$ hypothesis in Pesin's theory, Inst. Hautes Études Sci. Publ. Math. 59 (1984), 143-161.

[R] M. S. Raghunathan, Proof of Oseledec's multiplicative ergodic theorem, Israel J. Math. 32 (1979), 356-362.

[RS] D. Ruelle and M. Shub, Stable manifolds for maps, Global Theory of Dynamical Systems (Z. Nitecki and C. Robinson, Eds.), Lecture Notes in Math., vol. 819, Springer-Verlag, 1980, pp. 389-392.

[Ru] D. Ruelle, Characteristic exponents and invariant manifolds in Hilbert space, Ann. of Math. 115 (1982), 243-290.

[S] M. Shub, Global stability of dynamical systems, Springer, 1986.

$[\mathrm{K}, \mathrm{S}]^{*}$ A. B. Katok and J.-M. Strelcyn, Invariant manifolds, entropy and billiards: Smooth maps with singularities, Lecture Notes in Math., vol. 1222, Springer-Verlag, 1986.

Department of Mathematics, University of California, Berkeley, Berkeley, CaliforNIA 94720

Department of Mathematical Sciences, ibM Watson Research Center, Yorktown HEIGHTS, NEW YoRK 10598

* These lecture notes have only recently come to our attention. 